\title{
MEMORANDUM
}

No 39/2003

\section{Piecewise deterministic optimal control problems}

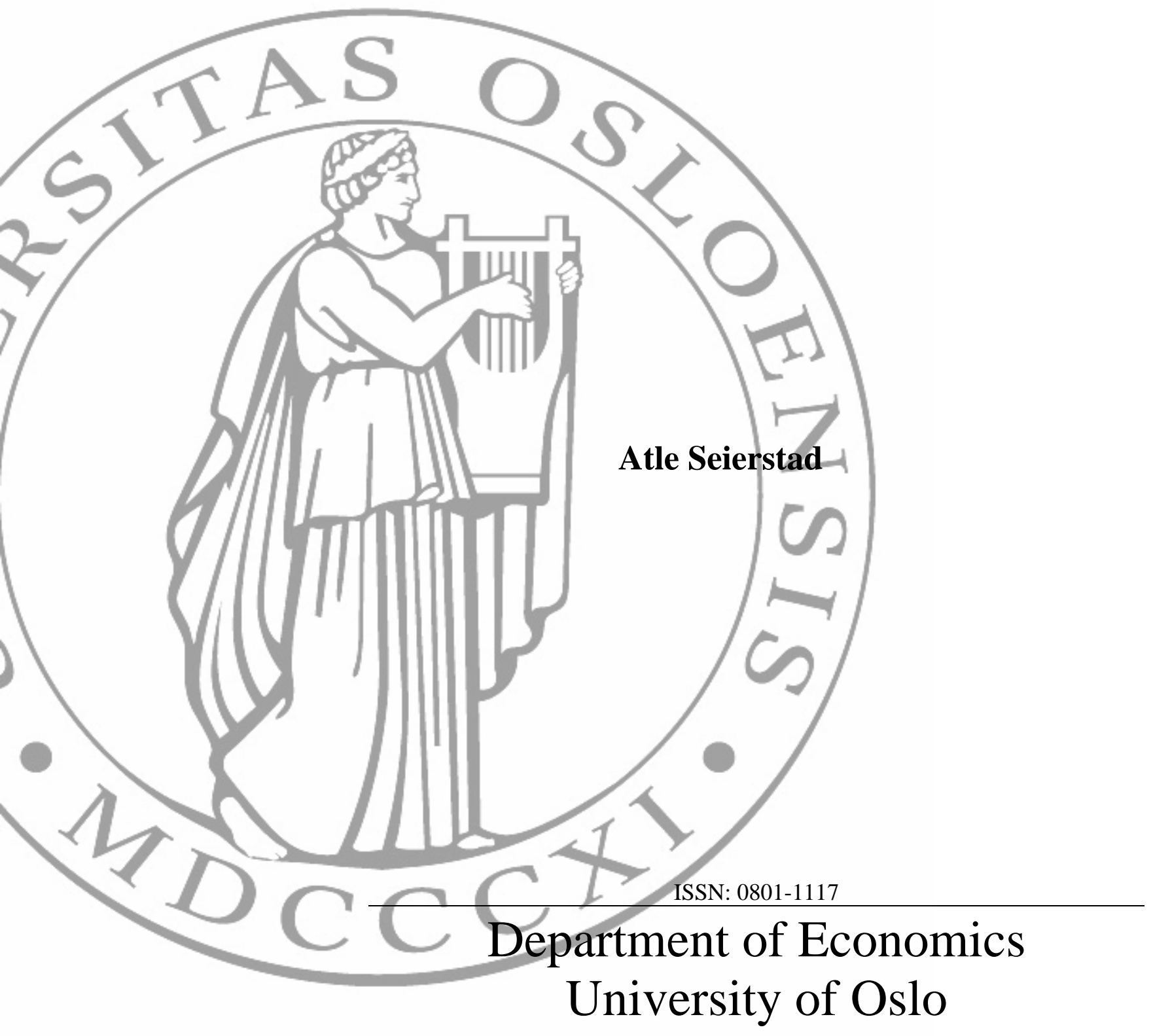


This series is published by the

University of Oslo Department of Economics

P. O.Box 1095 Blindern

N-0317 OSLO Norway

Telephone: + 4722855127

Fax: $\quad+4722855035$

Internet: http://www.oekonomi.uio.no/

e-mail: $\quad$ econdep@econ.uio.no
In co-operation with

\section{The Frisch Centre for Economic} Research

Gaustadalleén 21

N-0371 OSLO Norway

Telephone: $\quad$ +4722958820

Fax: $\quad$ +4722958825

Internet: http://www.frisch.uio.no/

e-mail: $\quad$ frisch@frisch.uio.no

List of the last 10 Memoranda:

\begin{tabular}{|ll|l|}
\hline No 38 & $\begin{array}{l}\text { Sheetal K. Chand } \\
\text { Risk, Return, and the Optimal Design of a Pension System. 26 pp. }\end{array}$ \\
\hline No 37 & $\begin{array}{l}\text { Rolf Aaberge, Ugo Colombino and Steinar Strøm } \\
\text { Do More Equal Slices Shrink the Cake? } \\
\text { An Empirical Investigation of Tax-Transfer Reform Proposals in Italy. } \\
\text { 31 pp. }\end{array}$ \\
\hline No 36 & $\begin{array}{l}\text { Kari Eika } \\
\text { Low Quality-Effective Demand. 43 pp. }\end{array}$ \\
\hline No 35 & $\begin{array}{l}\text { Karl Ove Moene } \\
\text { Social Democracy as a Development Strategy. 35 pp. }\end{array}$ \\
\hline No 34 & $\begin{array}{l}\text { Finn Roar Aune, Rolf Golombek and Sverre A. C. Kittelsen } \\
\text { Does Increased Extraction of Natural Gas Reduce Carbon Emissions?. } \\
\text { 40 pp. }\end{array}$ \\
\hline No 33 & $\begin{array}{l}\text { Christoph Schwierz } \\
\text { The Effects of Taxes and Socioeconomic Variables on Market Work and } \\
\text { Home Production in Norway in the Years 1970 to 2000. 61 pp. }\end{array}$ \\
\hline No 32 & $\begin{array}{l}\text { John K. Dagsvik, Steinar Strøm and Zhiyang Jia } \\
\text { A Stochastic Model for the Utility of Income. 39 pp. }\end{array}$ \\
\hline No 31 & $\begin{array}{l}\text { Karine Nyborg, Richard B. Howarth, and Kjell Arne Brekke } \\
\text { Green consumers and public policy: On socially contingent moral } \\
\text { motivation. 25 pp. }\end{array}$ \\
\hline No 30 & $\begin{array}{l}\text { Halvor mehlum } \\
\text { A Finer Point in Forensic Identification. 11 pp. }\end{array}$ \\
\hline No 29 & $\begin{array}{l}\text { Svenn-Erik Mamelund } \\
\text { Effects of the Spanish Influenza Pandemic of 1918-19 on Later Life } \\
\text { Mortality of Norwegian Cohorts Born About 1900. 30 pp. }\end{array}$ \\
\hline
\end{tabular}

A complete list of this memo-series is available in a PDF® format at: http://www.oekonomi.uio.no/memo/ 


\title{
Piecewise deterministic optimal control problems
}

by

\author{
Atle Seierstad, University of Oslo
}

Piecewise deterministic control problems are problems involving stochastic disturbances of a special type. In certain situations, in an otherwise deterministic control system, it may happen that the state jumps at certain stochastic points of time. Examples are sudden oil finds, or sudden discoveries of metal deposits. Similarly, in seemingly deterministic processes, the dynamics may suddenly change character: At certain stochastic points in time, the right-hand side of the differential equation governing the system changes form, such changes being effected by jumps in a (dummy) state variable. Examples of such phenomena are sudden inventions, sudden ecological disasters, earthquakes, floods, storms, fires, the sudden capture of a criminal, that suddenly change the prospects of the firm, the society, the agriculture, the criminal... .Several papers have discussed such problems, often using more or less ad hoc methods. (Sometimes it is possible to rewrite the problem so that deterministic control theory applies.) A systematic method for solving such problems, based on the HJB-equation (the Hamilton-JacobyBellman equation) for the problem, is presented in Davis (1993), "Markov Models and Optimization", and also briefly discussed below. In this paper a related method, closer to deterministic control theory, is presented first. It is easiest to apply to problems with a bound on the number of possible jumps.

Thus, the main purpose of this paper is to show how some piecewise deterministic optimal control problems can be solved by techniques similar to those used in deterministic problems. The paper includes statements of several theoretical results. Proofs are given for the results involving the HJBequation and fields of extremals, (for the HJB-equation, replicating the ones in Davis (1993)).

\section{Free end, fixed final time problems}

Consider the following control system:

$$
\dot{x}=f(t, x, u), t \in[0, T], \quad x(0)=x^{0} \in R^{n}, \quad u \in U \subset R^{r} .
$$

Here, the control region $U$, the initial point $x^{0}, f$ and the terminal time $T$ are fixed. The vector $u$ is a control, subject to choice in $U$. At certain jump time-points $\tau_{i}, 0<\tau_{1}<\tau_{2}<\ldots$, the state jumps according to

$$
x\left(\tau_{j}+\right)-x\left(\tau_{j}-\right)=g\left(\tau_{j}, x\left(\tau_{j}-\right)\right)
$$


where $g$ is a fixed function. (Here, $x\left(\tau_{j}+\right)=$ right limit, $x\left(\tau_{j}-\right)=$ left limit.) The points $\tau_{i}$ are random variables taking values in $[0, \infty)$. It will often be assumed that at most $N<\infty$ jump points $\tau_{j}$ can occur in $[0, T]$. The stochastic assumptions on the jump points $\tau_{i}$ are as follows: Given $\tau_{i-1}$, then $\tau_{i}, i>1$, is exponentially distributed in $\left[\tau_{i-1}, \infty\right)$ with parameter (intensity) $\lambda(i)>0$, so the density of $\tau_{i}=\tau \geq \tau_{i-1}$ is $\lambda(i) e^{\left(-\lambda(i)\left(\tau-\tau_{i-1}\right)\right)}$. Moreover, $\tau_{1}$ is exponentially distributed in $[0, \infty)$ with parameter $\lambda(1)$. It is assumed that $\sup _{i} \lambda(i)<\infty$. (In some remarks, these stochastic assumptions will be generalized.) The case where a maximum of $N$ jumps can occur is the case where $\lambda(i)=0, i>N$. The problem to be solved is:

$$
\max _{u(., .)} E\left[\int_{0}^{T} f_{0}(t, x, u) d t+\sum_{\tau_{j}<T} g_{0}\left(\tau_{j}, x\left(\tau_{j}-\right)\right)+h_{0}(x(T-))\right]
$$

subject to [1]. Here $f_{0}, g_{0}$ and $h_{0}$ are fixed functions, $f_{0}$ measures the running benefit obtained from the process, $h_{0}$ is a scrap value (or bequest function), and $g_{0}$ measures the benefit obtained at jump times. Write $\omega=$ $\left(\tau_{1}, \tau_{2}, \ldots\right), \tau_{j}<\tau_{j+1}$. The control functions $u(t, \omega)=u\left(t, \tau_{1}, \tau_{2}, \ldots\right)$, are, separately, piecewise and left-continuous in $t$ and piecewise and right-continuous in each $\tau_{j}$. The control functions are assumed to depend (only) on the history up till now, hence for any given $t, u(t, \omega)$ depends only on the $\tau_{i}$ 's having occurred, (i.e., $\tau_{i}<t$ ). Such controls are called non-anticipating. (In Remark 1 below, a more precise description of these controls are given.) The optimization problem is to maximize the criterion in [3] in the class of non-anticipating controls. We then imagine that $u$ equals $u(t, \omega)$ in [1] and [3], and that $x$ in [3] equals $x(t, \omega):=x^{u}(t, \omega)$, the solution of the equations in [1],[2], for $u=u(t, \omega)$. The function $x^{u}(t, \omega)$ is constructed by solving [1] successively on the intervals $\left(0, \tau_{1}\right),\left(\tau_{1}, \tau_{2}\right),\left(\tau_{2}, \tau_{3}\right), \ldots$, . First, [1] is solved on $\left(0, \tau_{1}\right)$, using $\left(0, x^{0}\right)$ as initial point. Next, [1] is solved on $\left(\tau_{1}, \tau_{2}\right)$ using $\left(\tau_{1}, x^{u}\left(\tau_{1}+, \omega\right)\right.$ as initial point. Then, [1] is solved on $\left(\tau_{2}, \tau_{3}\right)$, using $\left(\tau_{2}, x^{u}\left(\tau_{2}+, \omega\right)\right.$ as initial point, and so on. The initial states $\left.x^{u}\left(\tau_{1}+, \omega\right), x^{u}\left(\tau_{2}+, \omega\right), \ldots,\right)$ are all obtained from [2]. For convenience, the function $x(t, \omega)$ is taken to be leftcontinuous, (sometimes we write $x(t-, \omega)$ in certain formulas, although we could as well have written $x(t, \omega))$. The functions $x(t, \omega)$ become rightcontinuous in each $\tau_{j}$.

A solution methods will be presented in a moment. The method yields candidates for the optimal controls that come out in a slightly different form than $u(t, \omega)$. After presenting the method, the relationship between the various types of controls is discussed. 


\section{Remark 1 (Comment on non-anticipating controls)}

A more precise definition of non-anticipating controls is as follows: For any given $\omega=\left(\tau_{1}, \tau_{2}, \ldots\right)$ and $t$, let $i(t, \omega)$ be the largest index $i$ such that $\tau_{i}<t$. Then, $u(t, \omega)$ is called non-anticipating if for any $t$, any $\omega=\left(\tau_{1}, \tau_{2}, \ldots\right)$ and $\omega^{\prime}=\left(\tau_{1}^{\prime}, \tau_{2}^{\prime}, \ldots\right)$, the equality $u(t, \omega)=u\left(t, \omega^{\prime}\right)$ holds, whenever $i(t, \omega)=$ $i\left(t, \omega^{\prime}\right)$ and $\tau_{j}=\tau_{j}^{\prime}$ for $j \leq i(\omega, t)$.

When $t>\tau_{j}$, write $u\left(t, \omega^{j}\right)=u\left(t, \tau_{1}, \ldots, \tau_{j}, T, T+1, T+2, \ldots\right)$. A similar notation is used also in connection with other nonanticipating functions. Note that, as long as $t \in\left(\tau_{j}, \tau_{j+1}\right], u(t, \omega)=u\left(t, \omega^{j}\right)$.

\section{Solution method}

In this section, the functions $f, f_{0}, g_{0}, g$, and $h_{0}$ are assumed to be $C^{2}$. It is also assumed that the maximal number of jumps $N$ is finite. In this case, one standard method of solving the problem is as follows.

First, find a control $\hat{u}(t, x, p)$ such that

$$
\hat{u}(t, x, p) \text { maximizes } H(t, x, u, p):=f_{0}(t, x, u)+p f(t, x, u) \text { for } u \in U \text {. }
$$

(In this maximization, $t, x, p$ are just parameters in the problem.) Then let us write down the so-called characteristic equations. (Below $H_{x}, g_{0 x}, h_{0 x}$ denote gradients with respect to $x, g_{x}$, is a Jacobian matrix with respect to $x$, and $I$ is the identity matrix.)

$$
\begin{gathered}
\dot{x}(t)=f(t, x, \hat{u}(t, x, p)) \\
\dot{p}(t)=-H_{x}(t, x, \hat{u}(t, x, p), p)+\lambda(j+1) p \\
-\lambda(j+1)\left[g_{0 x}(t, x)+p(t ; t, x+g(t, x) ; j+1)\left(I+g_{x}(t, x)\right],\right.
\end{gathered}
$$

with boundary conditions

$$
x(s)=y, \quad p(T)=\left[h_{0 x}(x)\right]_{x=x(T ; s, y, j)}
$$


Here, the pair $(s, y)$ is arbitrary. The ordinary differential equations [5]-[7] are solved by backwards recursion: First [5]-[7] are solved on $[s, T]$ for $j=N$, in which case $\lambda(N+1)=0$ and [6] reduces to $\dot{p}(t)=-H_{x}(t, x, \hat{u}(t, x, p), p)$. The solution pair $x(),. p($.$) obtained is denoted x(t ; s, y, N), p(t ; s, y, N)$. Then [5]-[7] are solved on $[s, T]$ for $j=N-1$. In this case, the known function $p(. ; ., ., N)$ is inserted in [6]. The pair of solutions obtained is denoted $x(t ; s, y, N-1), p(t ; s, y, N-1)$. Then [5]-[7] are solved on $[s, T]$ for $j=N-2$, with $p(. ; ., ., N-1)$ inserted in [6]. And so on. The controls $u(t ; s, y, j):=$ $\hat{u}(t, x(t ; s, y, j), p(t ; s, y, j)), j=0,1, \ldots$, are our candidates for the optimal controls, (they yield non-anticipating candidates for optimality, see Remark 2 below).We call the solutions $x(t ; s, y, j), j=0,1,2, \ldots$ characteristic solutions, (and $u(t ; s, y, j), p(t ; s, y, j)$, characteristic controls, respectively characteristic adjoint functions). Finally, $(x(t ; s, y, j), p(t ; s, y, j)), j=0,1, \ldots$ are called characteristic pairs, and $(x(t ; s, y, j), u(t ; s, y, j), p(t ; s, y, j))$ characteristic triples. (Sometimes the word "extremal" are used instead of the word "characteristic".)

\section{Remark 2 Characteristic triples yield non-anticipating candidate controls}

Let us show that characteristic triples give rise to non-anticipating candidate controls, ("candidate" = candidate for optimality). For the given initial point $\left(0, x^{0}\right)$, the characteristic triple $x(t ; s, y, j), u(t ; s, y, j), p(t ; s, y, j)$ give rise to non-anticipating functions $x(t, \omega), u(t, \omega), p(t, \omega)$ : As always, $\omega=\left(\tau_{1}, \tau_{2}, \ldots\right)$. For $t \leq \tau_{1}, x(t, \omega)=x\left(t ; 0, x^{0}, 0\right)$. For $\tau_{1}<t \leq \tau_{2}$, $x(t, \omega)=x\left(t ; \tau_{1}, x\left(\tau_{1}-, \omega\right)+g\left(\tau_{1}, x\left(\tau_{1}-, \omega\right)\right), 1\right)$. Continuing in this manner, in general, we have that, for $\tau_{j}<t \leq \tau_{j+1}, x(t, \omega)=x\left(t ; \tau_{j}, x\left(\tau_{j}-, \omega\right)+\right.$ $\left.g\left(\tau_{j}, x\left(\tau_{j}-, \omega\right)\right), j\right)$. Furthermore, (for $\left.\tau_{j}<t \leq \tau_{j+1}\right)$,

$u\left(t ; \tau_{1}, \ldots, \tau_{j}\right):=\left(u\left(t ; \tau_{j}, x\left(\tau_{j}+, \omega\right), j\right), p\left(t ; \tau_{1}, \ldots, \tau_{j}\right):=p\left(t ; \tau_{j}, x\left(\tau_{j}+, \omega\right), j\right)\right.$. In this manner a triple $x(t, \omega), u(t, \omega), p(t, \omega)$ is obtained such that necessary conditions specified below (Theorem 1) are satisfied. Hence $u(t, \omega)$ is a candidate for optimality. If the collection $u(t ; s, y, j)$ gives rise to an optimal control $u^{*}(t, \omega)$ in problem [1]-[3], (which we may hope that it does!), we call the collection $u(t ; s, y, j), j=0,1, \ldots$ optimal in the problem.

Example 1 Consider the problem:

$$
\max E\left[\int_{0}^{T}-u^{2} / 2 d t+a x(T)\right], \quad \dot{x}=u \in \mathbb{R}, x(0)=0,
$$

with a possibility for a single, unit upwards jump in $x(t)$ at $\tau \in[0, \infty)$, with $\tau$ being exponentially distributed with intensity $\lambda$, (i.e. the jump point $\tau$ is 
distributed with density $\left.\lambda e^{-\lambda \tau}\right)$.

The maximum condition gives $\hat{u}=p$, and the characteristic equations for $j=1$ (i.e. after a jump) becomes $\dot{p}=0, \dot{x}=p$, with $p(T)=a$. Here, $t$ will denote running time. Hence, $u(t ; s, y, 1)=p(t ; s, y, 1)=a, x(t ; s, y, 1)=$ $y+a(t-s)$.

Next, for $j=0$, the characteristic equations become: $\dot{x}=p, \dot{p}=-\lambda a+\lambda p$, with $p(T ; s, y, 0)=a$. Then, $p(t ; s, y, 0)=a+C e^{\lambda t}$ where $C$ is determined by $p(T ; s, y, 0)=a$, so $C=0$, and $p(t ; s, y, 0)=a$. Thus, $u(t ; s, y, 0)=a$ is the control also before the jump, which should not come as a surprise. Sufficient conditions presented below (Theorem 2) secure that the optimal control has been found.

Next, replace $a x(T)$ in the criterion by $a x(T)^{2} / 2$, with $1-a T>0$.

As above, $\hat{u}=p, p(t ; s, y, 1)=p(T ; s, y, 1)$ and $x(t ; s, y, 1))=$ $y+p(T ; s, y, 1)(t-s)$. Now, $p(T ; s, y, 1)$ is determined by the condition that $a x(T ; s, y, 1)=a(y+p(T ; s, y, 1)(T-s))=p(T ; s, y, 1)$, which gives $p(T ; s, y, 1)=a y /(1+a(s-T))$, so $u(t ; s, y, 1)=a y /(1+a(s-T))$ and $x(t ; s, y, 1)=y+a y(t-s) /(1-a(T-s))$.

Let us find the characteristic solutions for $j=0$. The characteristic equations become $\dot{p}=-\lambda a(x+1) /(1+a(t-T))+\lambda p, \dot{x}=p$. From this pair of equations the following second order differential equation for $x$ follows:

$$
d^{2} x / d t^{2}=-\lambda a(x+1) /(1+a(t-T))+\lambda d x / d t .
$$

We prove below that this equation has the solution

$$
x(t ; s, y, 0)=-1+(C \beta(t)+D)(1+a(t-T)),
$$

where $\beta(t)=\int_{T}^{t} e^{\lambda \sigma} /(1+a(\sigma-T))^{2} d \sigma$ and where $C$ and $D$ are two arbitrary (integration) constants. The boundary conditions are $x(s ; s, y, 0)=y$ and $p(T ; s, y, 0)=a x(T ; s, y, 0)$, so, (recalling $\dot{x}=u=p$ ), we get $\dot{x}(T, s, y, 0)=$ $a x(T ; s, y, 0)$. Now, $[* *]$ yields that $\dot{x}(T, s, y, 0)$ equals $C \dot{\beta}(T)+(C \beta(T)+$ $D) a=C e^{\lambda T}+a D$, so the next to last equality becomes $C e^{\lambda T}+a D=$ $a(-1+D)$. From this equality and $x(s ; s, y, 0)=y$, the two integration constants can be determined for any $(s, y)$. Actually, before any jumps have happened, it suffices to find explicit formulas for $C$ and $D$ in terms of $(s, y)$, for $(s, y)=(0,0)$. In this case the two equalities become $C e^{\lambda T}+$ $a D=a(-1+D)$ and $x(0 ; 0,0,0)=-1+(C \beta(0)+D)(1-a T)=0$, 
from which $C$ and $D$ can be determined. The first equality immediately gives $C=-a e^{-\lambda T}$, and inserting this value for $C$ in the second ones gives $D=1 /[1-a T]+a e^{-\lambda T} \beta(0)$. The candidates for the optimal policy is hence to use $\left.u(t ; 0,0,0)=(d / d t)\left[-a e^{-\lambda T} \beta(t)+1 /\{1-a T\}+a e^{-\lambda T} \beta(0)\right](1+a(t-T))\right]$ as long as no jump has occurred. When a jump has occurred at some $\tau$, we use the control $u(t, \omega):=u(t, \tau):=u(t ; \tau, x(\tau-; 0,0,0)+1,1)=$ $a(x(\tau-; 0,0,0)+1) /(1+a(\tau-T))$ from then on, this control is constant in $t, t>\tau$ ). (Note that from the characteristic triples we have obtained nonanticipating controls.)

To prove [**], define $w(t)$ by $w(1+a(t-T))=x+1$. Then $(d w / d t)(1+a(t-T))+w a=d x / d t$ and $\left(d^{2} w / d t^{2}\right)(1+a(t-T))+2(d w / d t) a=d^{2} x / d t^{2}$, so $\left(d^{2} w / d t^{2}\right)(1+a(t-T))+2(d w / d t) a=-\lambda a(x+1) /(1+a(t-T))+\lambda d x / d t=$ $-\lambda a w+\lambda(d w / d t)(1+a(t-T))+\lambda a w$, and $d^{2} w / d t^{2}=[\lambda-2 a /(1+a(t-T))] d w / d t$. Integrating this separable equation, we get $d w / d t=C e^{\lambda t} /(1+a(t-T))^{2}$, so $w(t)=C \beta(t)+D$. Here $C$ and $D$ are two integration constants. By definition of $w(t), x(t ; s, y, 0)=$ $-1+w(t)(1+a(t-T))$ and $[* *]$ follows.

Do sufficient conditions apply here? Yes, but because the scrap value function is convex rather than concave, a theorem not using concavity is needed, in this example Theorem 3 below is applicable.

\section{Example 2}

$\max E\left\{\int_{0}^{1}-u^{2} / 2 d t+x(1)\right\}$, subject to $d x / d s=u \in \mathbb{R}, x(0)=0, x\left(\tau_{j}+\right)-$ $x\left(\tau_{j}-\right)=x\left(\tau_{j}-\right)$.

We assume that a maximum of $N<\infty$ jumps can occur, and that $\tau_{j}$ is exponentially distributed in $\left[\tau_{j-1}, \infty\right)$ with intensity $\lambda_{j}$, all $\lambda_{j}$ different. An interpretation of the problem might be that $x$ is the value of an investor's stock holding in a research firm, which now and then makes an invention. Each time an invention occurs, the price of the stock doubles. Moreover, any change in the stock (using $u \neq 0$ ) is costly. (The manner this is taken care of is debatable.) The best opportunities for inventions are exploited first, so we may imagine that $\lambda_{j}$ is a decreasing sequence.

Maximization of the Hamiltonian yields $\hat{u}=p$, hence $u(t ; s, y, j)=p(t ; s, y, j)$. It remains to find $p(t ; s, y, j)$. Now, $(d / d t) p(t ; s, y, N)=0$, so $p(t ; s, y, N)=$ $p(1 ; s, y, N)=1$. It is independent of the starting point $(s, y)$. This will also 
be seen to hold for $j<N$, so we drop $(s, y)$ in the symbols for the adjoint functions. Next, $(d / d t) p(t ; N-1)=-2 \lambda_{N} p(t ; N)+\lambda_{N} p(t ; N-1)$, which has the solution $p(t ; N-1)=2-e^{\lambda_{N}(t-1)}$. (To determine this solution also $p(1)=1$ has been used.) The formula is easily obtained by using $e^{-\lambda_{N}(t-1)}$ as integrating factor (i.e. by multiplying on both sides of the differential equation by this factor). The method is shown in detail in the next calculation. Let us find $p(t ; N-2)$ :

$(d / d t) p(t ; N-2)=-2 \lambda_{N-1} p(t ; N-1)+\lambda_{N-1} p(t ; N-2)=$ $-2 \lambda_{N-1} 2+2 \lambda_{N-1} e^{\lambda_{N}(t-1)}+\left(\lambda_{N-1}\right) p(t ; N-2)$.

Using $e^{-\left(\lambda_{N-1}\right)(t-1)}$ as integrating factor, i.e. multiplying on both sides by this factor, and rearranging, we get

$e^{-\lambda_{N-1}(t-1)} \cdot(d / d t) p(t ; N-2)-\lambda_{N-1} e^{-\lambda_{N-1}(t-1)} p(t ; N-2)=$ $-4 \lambda_{N-1} e^{-\left(\lambda_{N-1}\right)(t-1)}+2 \lambda_{N-1} e^{\left(\lambda_{N}-\lambda_{N-1}\right)(t-1)}$.

The left-hand side of the first equality equals $(d / d t)\left[e^{-(t-1)} p(t ; N-2)\right]$, so integrating on both sides yields

$e^{-\lambda_{N-1}(t-1)} p(t ; N-2)=$

$4 e^{-\left(\lambda_{N-1}\right)(t-1)}+2 \lambda_{N-1}\left(\lambda_{N}-\lambda_{N-1}\right)^{-1} e^{\left(\lambda_{N}-\lambda_{N-1}\right)(t-1)}+C$,

where $C$ is an integration constant. Multiplying by $e^{\lambda_{N-1}(t-1)}$ on both sides, and using $p(1, N-1)=1$ yield $p(t ; N-2)]=4+2 \lambda_{N-1}\left(\lambda_{N}-\lambda_{N}\right)^{-1} e^{\lambda_{N}(t-1)}+$ $\left(1-4-2 \lambda_{N-1}\left(\lambda_{N}-\lambda_{N-1}\right)^{-1}\right) e^{\lambda_{N-1}(t-1)}$. In general, we guess that $p(t ; N-j)$ has the following form: $p(t ; N-j)=2^{j}+\sum_{0 \leq i \leq j-1} a_{j}^{i} e^{\lambda_{N-i}(t-1)}$. Let us prove that $p(t ; N-(j+1))$ has a similar form. We have

$(d / d t) p(t ; N-(j+1))=-2 \lambda_{N-j} p(t ; N-j)+\lambda_{N-j} p(t ; N-(j+1))=$ $-2 \lambda_{N-j} 2^{j}-2 \lambda_{N-j} \cdot \sum_{0 \leq i \leq j-1} a_{j}^{i} e^{\lambda_{N-i}(t-1)}+\left(\lambda_{N-j}\right) p(t ; N-(j+1))$.

Using the integrating factor $e^{-\lambda_{N-j}(t-1)}$ in the differential equation for $p(t ; N-$ $(j+1))$ yields that $p(t ; N-(j+1))$ has the form: $p(t ; N-(j+1))=$ $2^{j+1}+\sum_{0 \leq i \leq j} a_{j+1}^{i} e^{\lambda_{N-i}(t-1),}$, were, for $i \leq j-1$,

$$
a_{j+1}^{i}=2 \lambda_{N-j} a_{j}^{i} /\left(\lambda_{N-j}-\lambda_{N-i}\right), i<j .
$$

The last coefficient in the expression for $p(t ; N-j), a_{j}^{j-1}$, is an integration constant. It is determined by $p(1 ; N-j))=1$, so 


$$
1-2^{j}-\sum_{0 \leq i \leq j-1} a_{j}^{i}=a_{j+1}^{j},
$$

Using [a] for $a_{j+1}^{i}, a_{j}^{i}, \ldots, a_{i+2}^{i}$, yields

$$
a_{j+1}^{i}=a_{i+1}^{i} \Pi_{i+1 \leq m \leq j} 2 \lambda_{N-m} /\left(\lambda_{N-m}-\lambda_{N-i}\right), \beta<j .
$$

The coefficients $a_{k+1}^{k}, k=0,1,2, \ldots, N-1$, are determined by the following difference equation, obtained by combining $[\mathrm{b}]$ and $[\mathrm{c}]$ :

$$
a_{k+2}^{k+1}=1-2^{k+2}-\sum_{0 \leq i \leq k} a_{i+1}^{i} \Pi_{i+1 \leq m \leq k+1} 2 \lambda_{N-m} /\left(\lambda_{N-m}-\lambda_{N-i}\right)
$$

First, as $p(t ; N-1)=2-e^{\lambda^{N}(t-1)}$, we get $a_{1}^{0}=-1$. Then, [d] gives us all $a_{k+1}^{k}, k=1,2,, \ldots, N-1$. By [c], for any $j, j<k-1$ we know all $a_{k}^{j}$. Thus, we know $p(t, N-j)=u(t, N-j)$. A sufficient condition based on concavity, (Remark 3 below), gives the optimality of $u(t, N-j), j=0,1,2, \ldots, N$, (or of the associated non-anticipating control $u(t, \omega))$.

In Remark 2, we saw that characteristic pairs give rise to non-anticipating controls. Let us give some comments on how so-called Markov controls arise.

\section{Remark 3 (Characteristic controls yield Markov candidate con- trols)}

When constructing the characteristic controls $u(t ; s, y, j)$ by the method above, we most often obtain that

$$
u\left(t^{\prime} ; s, y, j\right)=u\left(t^{\prime} ; t, x(t ; s, y, j), j\right), \quad t^{\prime}>t>s
$$

In fact, for any given $j$, when $\hat{u}(t, x, p)$ is unique and $C^{1}$, for any given initial point $(s,(y, \hat{p}))$ solutions to [5] and [6], are unique. Next, let $\hat{p}$ be a parameter to be determined by $p(T)=h_{0 x}(x(T ; s, y, j))$. Often this condition gives a unique value of $\hat{p}$. Under such circumstances, [8] will hold.

Let us discuss [8] a little more. Suppose that the solution start in $(s, y, j)$, (since $j$ is mentioned, it means that exactly $j$ jumps have already occurred in $[0, s))$. The control $u(t ; s, y, j)$ then (hopefully) describes the optimal behaviour from $s$ on. At any time $t^{\prime} \geq s$, the control value $u\left(t^{\prime} ; s, y, j\right)$ should be used as long as no further jumps occur. Let $x$ be a point that is reached 
by $x(. ; s, y, j)$ at time $t$, and assume that even at time $t$ only $j$ jumps have occurred. At a time $t^{\prime} \geq t$ we have then two prescriptions for which control value to use, namely $u\left(t^{\prime} ; s, y, j\right)$ and $u\left(t^{\prime} ; t, x, j\right)=u\left(t^{\prime} ; t, x(t ; s, y, j), j\right)$. These two values should coincide, and that is what [8] require them to do. Letting in particular $t^{\prime}=t=s$, then, assuming [8], we can say that $u(t, x, j):=u(t ; t, x, j)$ is the control value to use when we are at point $(t, x, j)$. A control of the form $u(t, x, j)$ is called a Markov control. Thus, most often, characteristic controls give rise to Markov candidate controls.

\section{Remark 4 (Non-anticipating-, characteristic-, and Markov controls)}

First, Markov controls and non-anticipating controls will be compared. Given that $j$ jumps have occurred at $\tau_{1}, \ldots, \tau_{j}$, a non-anticipating control $u(t, \omega)$ makes it possible to calculate the state $x$ at time $t$ by using [1] and [2]. At such a point $(t, x)$, the control function prescribes the control value to be $u(t ; \omega)$, or, "looking ahead", to be $u\left(t^{+}, \omega\right)$. Of course, even if different $\omega$ 's (i.e. different $\left.\tau_{1}, \ldots, \tau_{j}\right)$ by chance lead us to the same point $(t, x)$, different control values (depending on $\omega$ ) may be prescribed. However, the above system has the property that the future development of the system depends only on $t, x$ and $j$ and not when the jumps $\tau_{1}, \ldots, \tau_{j}$ have occurred. We can therefore expect optimal behaviour at time $s$ to depend only on $(t, x, j)$. Thus, an optimal control $u^{*}\left(t^{+}, \omega\right)$ will prescribe the same control value at $(t, x)$, irrespectively of how the system came to $x$ at time $t$, given that $j$ jumps have occurred. Hence, optimal controls can be expected to be of the type $u^{*}\left(t^{+}, x, j\right)$, i.e. Markov controls. We saw in Remark 3 that characteristic controls most often yield Markov controls, as the consistency condition [8] most often holds. They also yield non-anticipating controls, as we saw in Remark 2.

If Markov controls $u(t, x, j)$ are given, let us imagine that these are inserted into [1], which then are solved on $[s, T]$ for initial condition $(s, y)$. The solutions obtained are denoted $x(t ; s, y, j)$, (they satisfy $x(t ; s, y, j)=y$ ), and corresponding controls are $u(t ; s, y, j)=u(t, x(t ; s, y, j), j)$. (Normally they automatically satisfy the consistency condition [8].) As in Remark 2, solutions $x(T, s, y, j)$ give rise to nonanticipating solutions $x(t, \omega)$, and then also to non-anticipating controls $u(t, \omega)\left(=u(t, x(t, \omega), j), t \in\left(t_{j, j+1}\right]\right.$.

The solution method above yields characteristic controls $u(t ; s, y, j)$, which also give rise to non-anticipating candidate controls $u(t, \omega)$ with corresponding solutions $x(t, \omega)$. 


\section{Remark 5 (Explicit dependence on $\mathrm{j}$ )}

The functions $f_{0}, f, g_{0}, g$ can be allowed to depend explicitly on $j$. In $f_{0}$ and $f, j$ has value 0 as long as no jump has occurred, (i.e. in $\left.\left(0, \tau_{1}\right)\right)$, the value 1 when exactly one jump has occurred, (i.e. in $\left(\tau_{1}, \tau_{2}\right)$ ), the value 2 when exactly two jumps have occurred (i.e. in $\left(\tau_{2}, \tau_{3}\right)$ ), and so on. To simplify some statements, let $\tau_{0}=0, \omega^{0}=0$, and let $\omega^{j}:=\left(\tau_{0}, \ldots, t_{j}\right)$. The differential equation is thus

$$
\dot{x}=f(t, x, u, j), t \in\left(\tau_{j}, \tau_{j+1}\right), x(0+)=x^{0}, u \in U,
$$

the jump condition is, for $j>0$,

$$
x\left(\tau_{j}+\right)-x\left(\tau_{j}-\right)=g\left(\tau_{j}, x\left(\tau_{j}-\right), j\right),
$$

and the criterion is

$$
E\left[\sum_{j} \int_{\min \left\{T, \tau_{j}\right\}}^{\min \left\{T, \tau_{j+1}\right\}} f_{0}(t, x, u, j) d t+\sum_{\tau_{j}<T} g_{0}\left(\tau_{j}, x\left(\tau_{j}-\right), j\right)+h_{0}(x(T-))\right] .
$$

The solution procedure now uses the four relationships:

$\hat{u}(t, x, p, j)$ maximizes $H(t, x, u, p, j):=f_{0}(t, x, u, j)+p f(t, x, u, j)$ for $u \in U$.

$$
\dot{x}(t)=f(t, x, \hat{u}(t, x, p, j), j)
$$

$$
\begin{gathered}
\dot{p}(t)=-H_{x}(t, x, \hat{u}(t, x, p, j), p, j)+\lambda(j+1) p \\
-\lambda(j+1)\left[g_{0 x}(t, x, j+1)+p(t ; t, x+g(t, x, j+1), j+1)\left(I+g_{x}(t, x, j+1)\right)\right],
\end{gathered}
$$

with boundary conditions

$$
x(s)=y, p(T)=\left[h_{0 x}(x)\right]_{x=x(T ; s, y, j)} .
$$

From now on, this explicit dependence on $j$ will be assumed. We shall also 
require that for a pair $x(t, \omega), u(t, \omega)$ to be called admissible, the inequalities $\sup _{t, \omega}|x(t, \omega)|<\infty$ and $\sup _{t, \omega}|u(t, \omega)|<\infty$ have to be satisfied.

\section{Precise necessary conditions and sufficient conditions}

Let "v.e." (virtually everywhere ) mean everywhere except a finite (or countable ) number of points, and let a.s. mean almost surely, i.e. with probability 1 .

Theorem 1 (Necessary condition, free end) Assume that $x^{*}(t, \omega), u^{*}(t, \omega)$, is an optimal pair in problem [1]-[3], such that $u^{*}(t, \omega)$ is bounded. Then, for each $\omega$, there exists a non-anticipating function $t \rightarrow p(t, \omega)$, continuous in $t$ between the points $\tau_{i}$ in $\omega$, differentiable v.e., such that, a.s., for v.e. $t>\tau_{j}, j=0,1,2, \ldots$,

$$
\begin{gathered}
u \rightarrow f_{0}\left(t, x^{*}\left(t, \tau_{1}, \ldots, \tau_{j}\right), u, j\right)+ \\
p\left(t, \tau_{1}, \ldots, \tau_{j}\right) f\left(t, x^{*}\left(t, \tau_{1}, \ldots, \tau_{j}\right), u, j\right)
\end{gathered}
$$

has a maximum at $u^{*}\left(t, \tau_{1}, \ldots, \tau_{j}\right)$ in $U$.

Moreover, $t \rightarrow p\left(t, \tau_{1}, \ldots, \tau_{j}\right), t>\tau_{j}$, satisfies

$$
\begin{gathered}
\dot{p}\left(t, \tau_{1}, \ldots, \tau_{j}\right)=-f_{0 x}\left(t, x^{*}\left(t, \tau_{1}, \ldots, \tau_{j}\right), u^{*}\left(t, \tau_{1}, \ldots, \tau_{j}\right), j\right) \\
-p\left(t, \tau_{1}, \ldots, \tau_{j}\right) f_{x}\left(t, x^{*}\left(t, \tau_{1}, \ldots, \tau_{j}\right), u^{*}\left(t, \tau_{1}, \ldots, \tau_{j}\right), j\right)- \\
\lambda(j+1)\left[g_{0 x}\left(t, x^{*}\left(t, \tau_{1}, \ldots, \tau_{j}\right), j+1\right)+p\left(t+, \tau_{1}, \ldots, \tau_{j}, t\right)\{I+\right. \\
\left.\left.g_{x}\left(t, x^{*}\left(t, \tau_{1}, \ldots, \tau_{j}\right), j+1\right)\right\}\right]+\lambda(j+1) p\left(t, \tau_{1}, \ldots, \tau_{j}\right) . \\
p\left(T, \tau_{1}, \ldots \tau_{j}\right)=h_{0 x}\left(x^{*}\left(T-, \tau_{1}, \ldots \tau_{j}\right)\right) .
\end{gathered}
$$

(As explained earlier, $x^{*}\left(t, \tau_{0}, \ldots, \tau_{j}\right)=x^{*}\left(t, \tau_{0}, \ldots, \tau_{j}, T, T+1, \ldots\right)$, and similarly for the $p$-function.) The function $p\left(t, \tau_{1}, \ldots, \tau_{j}\right), t>\tau_{j}$, is, separately, piecewise and left-continuous in $t$, and piecewise and right-continuous in each $\tau_{i}, i \leq j$.

Let us state precise conditions for Theorem 1 to hold. (See Remark 5 and Remark 3 in Seierstad (2002)). It suffices to assume that $f_{0}, f, g_{0}, g, h_{0}, f_{0 x}$, $f_{x}, g_{0 x}, g_{x}, h_{0 x}$, (exist and) are all, separately, piecewise continuous in $t$, and separately continuous in $x$ and in $u$. The five functions $f_{0}, f, g_{0}, g, h_{0}$ are Lipschitz continuous with respect to $x$ with a common $\operatorname{rank} \kappa^{n}, n=1,2, \ldots<\infty$, independent of $(t, u, j), u \in U \cap B(0, n)$. The five functions satisfy an inequality of the following form: For some constants $\alpha_{n}, \kappa_{n}$

$$
|\phi(t, x, u, j)| \leq \alpha_{n}+\kappa_{n}|x|, \text { for all }(t, x, u, j), u \in U \cap B(0, n) .
$$


Finally, $\sup _{j} \lambda(j)<\infty$. Now, $N$ may equal $\infty$.

If these conditions hold even for $n=\infty$, then in the theorem, $u^{*}(.,$.$) need not$ be bounded. If $N<\infty$ and $\alpha_{n}=\alpha, \kappa_{n}=\kappa^{n}=\kappa$ for all $n$, then $f_{0}, f, g_{0}, g, h_{0}$ need only be defined on $B(0, \Xi)$, where

$\Xi:=1+\left\{\left[\alpha+(1+\kappa)\left(\alpha e^{\kappa T} / \kappa\right)\right] / \kappa+\left|x^{0}\right|\right\}(1+\kappa)^{N+1} e^{\kappa T}$, and all properties in the preceding paragraph need only hold for $x$ in $B(0, \Xi)$. An alternative set of conditions in case $N<\infty$ is that, (for each $j$ ), $f_{0}, f, g_{0}$, $g, h_{0}, f_{0 x}, f_{x}, g_{0 x}, " g_{x}, h_{0 x}$, are continuous functions, and that $\sup _{t, \omega}\left|x^{*}(t, \omega)\right|<$ $\infty$.

From now on, these conditions are assumed. (Exceptions in certain remarks are explicitly stated.)

Formally, to solve a control problem completely, one has to find all possible controls $u^{* *}(t, \omega)$ satisfying the necessary conditions in Theorem 1. If one such candidate $u^{* *}(t, \omega)$ is constructed from characteristic controls, then, in many problems, one can prove that no other $u^{* *}(t, \omega)$ satisfies [16]-[18], i.e. there is a unique candidate for optimality. If one knows that the problem must have an optimal control, then it must be this unique candidate. (We have not provided results concerning existence of optimal controls, they are, however, similar to the Fillipov-Cesari results in deterministic control theory.)

Connected with this necessary condition is a sufficient condition, based on concavity: Define $\hat{H}(t, x, p, j)=\sup _{u \in U} H(t, x, u, p, j)$. Then the following theorem holds.

Theorem 2 (Sufficient condition based on concavity) Suppose the triple $\left(x^{*}(t, \omega), u^{*}(t, \omega), p(t, \omega)\right)$ satisfies the necessary conditions [16]-[18], with $\left(\left(x^{*}(t, \omega), u^{*}(t, \omega)\right)\right.$ satisfying [9], [10]). Suppose, furthermore, that $x \rightarrow h_{0}(x)$ and $x \rightarrow g_{0}(t, x, j)$ are concave, and, for each $j, \omega^{j}, t>\tau_{j}, x \rightarrow$ $\hat{H}\left(t, x, p\left(t, \omega^{j}\right), j\right)$, and $x \rightarrow p\left(t, \omega^{j}\right) g(t, x, j)$ are concave. Then $\left(x^{*}(t, \omega), u^{*}(t, \omega)\right)$ is optimal.

The concavity condition on $\hat{H}\left(t, x, p\left(t, \omega^{j}\right), j\right)$ holds in particular when $(x, u) \rightarrow$ $H\left(t, x, u, p\left(t, \omega^{j}\right), j\right):=f_{0}(t, x, u, j)+p\left(t, \omega^{j}\right) f(t, x, u, j)$ is concave. 


\section{Remark 6 (Sufficient concavity conditions for characteristic con- trols, $N<\infty)$}

Similar to Theorem 2, sufficient for the characteristic triple $x(t ; s, y, j), u(t ; s, y, j)$, $p(t ; s, y, j)$ to yield an optimal non-anticipating control is concavity of $x \rightarrow$ $g_{0}(t, x, j), x \rightarrow h_{0}(x), x \rightarrow \hat{H}(t, x, p(t ; s, y, j), j)$, and $x \rightarrow p(t ; s, y, j) g(t, x, j)$ for all $(s, y, j)$.

Above, we have dropped stating assumptions concerning the dependence of $x(t ; s, y, j), u(t ; s, y, j)$, and $p(t ; s, y, j)$ on $t, s$, and $y$. To a large extent, we can avoid stating explicitly such assumptions, by saying that, implicitly, (for example in the preceding remark), it is assumed that the nonanticipating triples $(x(t, \omega), u(t, \omega), p(t, \omega))$ arising from the characteristic triples satisfy the standard assumptions. It is implicit in what was said above that $t \rightarrow(x(t, s, y, j), p(t ; s, y, j))$ is continuous in $t \in[s, T]$ and that $t \rightarrow(\dot{x}(t, s, y, j), \dot{p}(t ; s, y, j))$ is piecewise continuous in $t \in(s, T)$.

When at most $N$ jumps can occur, there is a recursive procedure related to the necessary conditions. Loosely speaking, first $\left(x^{*}\left(t ; \tau_{1}, \ldots, \tau_{N}\right)\right.$, $\left.u^{*}\left(t ; \tau_{1}, \ldots, \tau_{N}\right), p\left(t ; \tau_{1}, \ldots, \tau_{N}\right)\right)$ is constructed, then $\left(x^{*}\left(t ; \tau_{1}, \ldots, \tau_{N-1}\right)\right.$, $\left.u^{*}\left(t ; \tau_{1}, \ldots, \tau_{N-1}\right), p\left(t ; \tau_{1}, \ldots, \tau_{N-1}\right)\right)$, and so on. One way of systematizing this procedure, was show in the solution method connected with [4]-[6] above.

Now, Theorem 1 also holds if there is no bound on the number of jumps that can occur, $(N=\infty)$. But, in this case no recursive procedure is available . Still, in principle, one seeks triples $\left(x^{*}\left(t ; \tau_{1}, \ldots, \tau_{j}\right), u^{*}\left(t ; \tau_{1}, \ldots, \tau_{j}\right), p\left(t ; \tau_{1}, \ldots, \tau_{j}\right)\right)$ satisfying the necessary conditions for $j=0,1,2, \ldots$. Sometimes, it may pay instead to turn to the so-called HJB-equation described later on.

The next theorem gives a sufficient condition not requiring concavity. The following definitions are needed: Let

$$
\begin{aligned}
& Q_{x(. .)}(j):=\left\{\left(t, x\left(t, \omega^{j}\right)\right): t \in\left(\tau_{j}, T\right), \text { for some } \omega^{j}\right), \\
& Q(j)=\cup_{x(. .)} Q_{x(. . .)}(j)
\end{aligned}
$$

the union taken over all admissible solutions $x(.,$.$) . Thus Q(j)$ consist of all points $(s, y)$ that can be reached after $j$ jumps, considering all possible admissible solutions and spacings of these $j$ jumps.

In the next theorem also sets $Q^{0}(j) \subset(0, T) \times \mathbb{R}^{n}$ appear, being (for each 
$j$ ) larger than $Q(j)$. Sometimes, these sets can be constructed by taking the union of $Q_{x(. .)}(j)$ for all solutions $x(.,$.$) , starting at time 0$ at given start points $\hat{x}^{0}$, where $\hat{x}^{0}$ runs through a (perhaps small) open ball around $x^{0}$. In other cases, $Q^{0}(j)$ can, for example, be the whole set $(0, T) \times \mathbb{R}^{n}$.

\section{Theorem 3 (Sufficiency based on a field of characteristic solutions)}

Assume that a maximum of $N$ jumps can occur. Assume that $h_{0}, f_{0}, f, g_{0}$ and $g$ are $C^{2}$-functions, and that the function $\hat{u}(t, x, p, j)$ (see [12]) is $C^{0} \times C^{1} \times C^{1}$ in an open set $Q^{*}(j)$. Assume that open sets $Q^{0}(j), Q^{0}(j)$ containing $Q(j)$, and solutions $x(t ; s, y, j), p(t ; s, y, j)$ of [13]-[15], (the characteristic equations with boundary conditions), have been found for $(s, y)=\left(0, x^{0}\right)$ in case $j=0$, and for any $(s, y) \in Q^{0}(j)$ and any $j$, being $C^{1} \times C^{2}$ in $(s, y)$ for $(s, y) \in Q^{0}(j)$, for any $t \in(s, T]$, and any $j$. Moreover, assume that $p(s ; s, y, j)$ and $p_{y}(s ; s, y, j)$ are bounded on bounded subsets of $Q^{0}(j)$. Assume that $(t, x(t ; s, y, j), p(t ; s, y, j))$ belongs to $Q^{*}(j)$ for all $t \in[s, T]$, and $(t, x(t ; s, y))$ belongs to $Q^{0}(j)$ for all $(s, y) \in Q^{0}(j)$, all $t \in(s, T)$, and that if $(s, y) \in Q^{0}(j)$, then $(s, y+g(s, y, j+1)) \in Q^{0}(j+1)$. Moreover, assume the following "consistency condition": For any $(s, y)$ in $Q(j)$ and $t \in(s, T]$,

$$
\begin{aligned}
& \left(x\left(t ; s^{\prime}, x\left(s^{\prime} ; s, y, j\right), j\right), p\left(t ; s^{\prime}, x\left(s^{\prime} ; s, y, j\right), j\right)\right)= \\
& (x(t ; s, y, j), p(t ; s, y, j)), \text { for all } s^{\prime} \in(s, t) .
\end{aligned}
$$

Furthermore, assume that $\hat{H}(s, x, p, j)$ is $C^{0} \times C^{2} \times C^{2}$ in $Q^{*}(j)$. Finally, assume that

$$
\hat{u}(t, x, p, j) \text { is bounded on bounded subsets of } Q^{*}(j) \text {. }
$$

Define $u(t ; s, y, j)=\hat{u}(t ; x(t ; s, y, j), p(t ; s, y, j), j)), j=0, \ldots, N$. Then $u(t ; s, y, j)$ : $j=0, \ldots, N$ are optimal controls.

In fact, in certain cases, the $C^{1} \times C^{2}$ of $(s, y) \rightarrow(x(t ; s, y, j), p(t ; s, y, j))$ automatically holds, see Remark 28 below.

While the results in the theorem do follow from the stated premisses, note that, hidden in the premisses is the fact that, in order for the stated differentiability of $(s, y) \rightarrow(x(t ; s, y, j), p(t ; s, y, j))$ to hold, the system must, in most cases, satisfy further differentiability properties (see Remark 28 below).

This theorem does not require concavity assumptions. So why use the preceding Theorem 2 (or Remark 6) at all? The answer is that when concavity holds, these tools are easier to apply, and in particular, there may be situations where concavity holds, but where it is impossible to find open sets $Q^{0}(j)$ and entities $x(t ; s, y, j), p(t ; s, y, j)$ satisfying all conditions in Theorem 
3. (Note than in a latter remark, (Remark 19), the conditions on the dependence of $x(t ; s, y, j), p(t ; s, y, j)$ on $(s, y)$ are slightly weakened.)

Example 1 revisited Consider the case of the scrap value function $a x(T)^{2} / 2$. Let $Q^{0}(j)=(0, T) \times \mathbb{R}$ for $j=0,1$. In Example 1, in this case, functions $x(t ; s, y, j), p(t ; s, y, j), j=0,1$ were found for each $(s, y)$, having the required $C^{1}$-property. Moreover, $\hat{H}$ and $\hat{u}$ are $C^{2}$, and [21] and [22] hold. Hence, $u(t ; s, y, j), j=0,1$ is an optimal collection.

Connected with Theorem 3, there is a sufficient condition that involves solutions of a sequence of partial differential equation, the HJB-equations of the problem. In fact, the solutions $(x(t ; s, y, j), p(t ; s, y, j))$ yield the solutions to these equations. A discussion of this theme is postponed to the next section on end constrained problems.

\section{Remark 7 (Time and state dependent jump intensity)}

Assume that $\lambda(j)$ is a $C^{1}$-function of $(t, x)$. Then the density of $\tau=\tau_{j} \in$ $\left[\tau_{j-1}, \infty\right)$ is

$$
\lambda(\tau, x(\tau), j) e^{\int_{\tau_{j-1}}^{\tau}-\lambda(\sigma, x(\sigma), j) d \sigma},
$$

where $x(s)=x(s, \omega)$. Now, an auxiliary state variable $z$ is needed, together with a modified adjoint equation:

$$
\begin{aligned}
& d z / d t=\lambda(t, x, j+1)\left\{z-g_{0}(t, x, j+1)-z(t ; t, x+g(t, x, j+1), j+1)\right\}- \\
& \quad f_{0}(t, x, \hat{u}(t, x, p), j),
\end{aligned}
$$

$$
\begin{gathered}
d p / d t=-H_{x}(t, x, \hat{u}(t, x, p), p, j)+\lambda(t, x, j+1) p- \\
\lambda(t, x, j+1)\left[g_{0 x}(t, x, j+1)+p(t, t, x+g(t, x, j+1), j+1)\left(I+g_{x}(t, x, j+1)\right)\right]- \\
\lambda_{x}(t, x, j+1)\left\{g_{0}(t, x, j+1)+z(t, t, x+g(t, x, j+1), j+1)-z\right\}
\end{gathered}
$$

Instead of finding characteristic pairs satisfying [5],[6] for $N, N-1, N-$ $2, \ldots$, one now has to find characteristic triples $x(t ; s, y, j), p(t ; s, y, j), z(t ; s, y, j)$, for $j=N, N-1, \ldots$ that satisfy simultaneously [5], [23] and [24], with $x(s)=y, p(T)=h_{0 x}(x(T)), z(T)=h_{0}(x(T))$. 


\section{Remark 8 (Stochastic jump sizes)}

A stochastic disturbance $V_{j} \in \mathbb{R}^{r^{*}}$ may be included in $g_{0}(t, x, j)$ and $g(t, x, j)$. Thus, we now write $g_{0}\left(t, x, V_{j}, j\right)$ and $g\left(t, x, V_{j}, j\right)$. Here $g$ and $g_{0}$ are, separately, $C^{2}$ in $(t, x)$, and the functions, as well as their first and second derivatives with respect to $t$ and $x$ are, separately, continuous in $v$. Thus at the jump times $\tau_{j}$, the stochastic variable $V_{j}$ influences $g_{0}$ and $g$, so [2] now reads $x\left(\tau_{j}+\right)-x\left(\tau_{j}-\right)=g\left(\tau_{j}, x(\tau-), V_{j}, j\right)$. If the stochastic variable $V_{j}$ has a distribution which depends on $\tau_{j}$, then the solution method connected with [5]-[7] again works when $N<\infty$, though in [6] we replace

$$
g_{0 x}(t, x, j+1)+p(t ; t, x+g(t, x, j+1), j+1)\left(I+g_{x}(t, x, j+1)\right)
$$

by

$$
\begin{gathered}
E\left[g_{0 x}\left(t, x, V_{j+1}, j+1\right)+\right. \\
\left.p\left(t ; t, x+g\left(t, x, V_{j+1}, j+1\right) ; j+1\right)\left(I+g_{x}\left(t, x, V_{j+1}, j+1\right)\right) \mid t\right]
\end{gathered}
$$

(The expectation is with respect to $V_{j+1}$ ).

If the distribution of $V_{j}$ depends on all $\tau_{i}, i \leq j$, and all earlier outcomes $v_{i}, i<j$, then in $\omega$, we now include all outcomes of the $V_{j}$ 's, so $\omega=$ $\left(\tau_{0}, v_{0}, \tau_{1}, v_{1}, \tau_{2}, v_{2}, \ldots\right),\left(v_{0}\right.$ having no effect), and non-anticipating entities at time $t$ now depend on the outcomes $\left(\tau_{i}, v_{i}\right)$ having occurred before $t$, e.g. $p(t, \omega)$ now equals $p\left(t ; \tau_{1}, v_{1}, \ldots \tau_{j}, v_{j}\right)$ when $\tau_{j}<t \leq \tau_{j+1}$. Assume that [19] holds for all $V_{j}$, for $\phi=g_{0}, g$. The necessary conditions of Theorem 1 still apply, provided we in [17] replace

$g_{0 x}\left(t, x^{*}\left(t, \tau_{1}, \ldots, \tau_{j}\right), j+1\right)+p\left(t+, \tau_{1}, \ldots, \tau_{j}, t\right)\left\{I+g_{x}\left(t, x^{*}\left(t, \tau_{1}, \ldots, \tau_{j}\right), j+1\right)\right\}$

by

$$
\begin{gathered}
E\left[g_{0 x}\left(t, x^{*}\left(t, \tau_{1}, v_{1}, \ldots, \tau_{j}, v_{j}\right), V_{j+1}, j+1\right)+\right. \\
p\left(t+, \tau_{1}, v_{1}, \ldots, \tau_{j}, v_{j}, t, V_{j+1}\right)\{I+ \\
\left.\left.\left.g_{x}\left(t, x^{*}\left(t, \tau_{1}, v_{1}, \ldots, \tau_{j}, v_{j}\right), V_{j+1}, j+1\right)\right\} \mid \tau_{1}, v_{1}, \ldots, \tau_{j}, v_{j}, t\right)\right]
\end{gathered}
$$

The function $p(t, \omega)$ is, separately, piecewise and left-continuous in $t$, and is piecewise and right-continuous in each $\tau_{j}$, and in each $v_{j}$, when the expectation $E\left[\alpha\left(V_{\tau_{j+1}}\right) \mid \tau_{1}, v_{1}, \ldots, \tau_{j}, v_{j}, \tau_{j+1}\right]$ is continuous in $\tau_{1}, v_{1}, \ldots, \tau_{j}, v_{j}, \tau_{j+1}$ for any piecewise and right-continuous function $\alpha\left(V_{\tau_{j+1}}\right)$. 


\section{Remark 9 (Reformulation of the necessary conditions)}

Define $\mu\left((t, \infty) \mid \tau_{0}, \ldots, \tau_{j}\right):=\exp \left[-\lambda(j+1)\left(t-\tau_{j}\right)\right], \mu\left(t \mid \tau_{0}, \ldots, \tau_{j}\right):=1-$ $\mu\left((t, \infty) \mid \tau_{0}, \ldots, \tau_{j}\right), j=0,1,2, \ldots .,\left(\tau_{0}=0\right)$, and $p^{*}\left(t, \tau_{1}, \ldots, \tau_{j}\right)=$ $\mu\left((t \infty) \mid \tau_{0}, \ldots, \tau_{j}\right) p\left(t, \tau_{1}, \ldots, \tau_{j}\right)$. Let $D \mu$ denote the density of the cumulative distribution $\mu$, and let a dot above a function mean a derivative with respect to time $t$. Then $p^{*}\left(t, \tau_{1}, \ldots, \tau_{j}\right)$ satisfies the differential equation [27] below. In fact the necessary conditions [16]-[18] yield the following necessary conditions:

Assume that $x^{*}(t, \omega), u^{*}(t, \omega)$ is an optimal pair in problem [9]-[11]. Then, there exists a non-anticipating function $p^{*}(t, \omega)$, such that for all $\omega, t \rightarrow$ $p^{*}(t, \omega)$ is continuous between the points $\tau_{i}$ in $\omega$, and such that for $\Lambda_{0}=1$, a.s., for v.e. $t>\tau_{j}$,

$$
\begin{aligned}
& u \rightarrow\left[\Lambda_{0} f_{0}\left(t, x^{*}\left(t, \tau_{1}, \ldots, \tau_{j}\right), u, j\right) \mu\left((t, \infty) \mid \tau_{0}, \ldots, \tau_{j}\right)+\right. \\
& \left.p^{*}\left(t, \tau_{1}, \ldots, \tau_{j}\right) f\left(t, x^{*}\left(t, \tau_{1}, \ldots, \tau_{j}\right), u, j\right)\right] D \mu\left(\tau_{0}, \ldots, \tau_{j}\right)
\end{aligned}
$$

has a maximum at $u^{*}\left(t, \tau_{1}, \ldots, \tau_{j}\right)$ in $U$.

Moreover, (still for $\Lambda_{0}=1$ ), for each $\omega, t \rightarrow p^{*}\left(t, \tau_{1}, \ldots, \tau_{j}\right), t>\tau_{j}$ is differentiable v.e., and satisfies v.e.

$$
\begin{gathered}
\dot{p}^{*}\left(t, \tau_{1}, \ldots, \tau_{j}\right)= \\
-\Lambda_{0} f_{0 x}\left(t, x^{*}\left(t, \tau_{1}, \ldots, \tau_{j}\right), u^{*}\left(t, \tau_{1}, \ldots, \tau_{j}\right), j\right) \mu\left(\left(t, \infty \mid \tau_{0}, \ldots, \tau_{j}\right)\right. \\
-p^{*}\left(t, \tau_{1}, \ldots, \tau_{j}\right) f_{x}\left(t, x^{*}\left(t, \tau_{1}, \ldots, \tau_{j}\right), u^{*}\left(t, \tau_{1}, \ldots, \tau_{j}\right), j\right)- \\
\Lambda_{0} g_{0 x}\left(t, x^{*}\left(t, \tau_{1}, \ldots, \tau_{j}\right), j+1\right) \dot{\mu}\left(t \mid \tau_{0}, \ldots, \tau_{j}\right)- \\
p^{*}\left(t+, \tau_{1}, \ldots, \tau_{j}, t\right)\left\{I+g_{x}\left(t, x^{*}\left(t, \tau_{1}, \ldots, \tau_{j}\right), j+1\right)\right\} \dot{\mu}\left(t \mid \tau_{0}, \ldots, \tau_{j}\right) .
\end{gathered}
$$

Finally,

$$
p^{*}\left(T, \tau_{1}, \ldots \tau_{j}\right)=h_{0 x}\left(x^{*}\left(T-, \tau_{1}, \ldots \tau_{j}\right)\right) \mu\left((T, \infty) \mid \tau_{0}, \ldots, \tau_{j}\right) .
$$

Here, $p^{*}(t, \omega)$ is, separately, piecewise and left-continuous in $t$, and piecewise and right-continuous in each $\tau_{j}$. 


\section{Remark 10 (General distribution of jump times)}

The necessary conditions in Remark 9 also hold, if the probability distribution of the $\tau$ 's are not of the type above. In fact, the above necessary conditions [27]-[29] hold for any given sequence of conditional densities $\dot{\mu}\left(t \mid \tau_{0}, \ldots, \tau_{j}\right), j=0,1, \ldots$, (for $j=0$, the density is simply $\left.\dot{\mu}(t)=\dot{\mu}\left(t \mid \tau_{0}\right)\right)$. If $N=\infty$, for the necessary conditions to hold, a condition ([29*] below) is needed. Define $\Theta_{m}\left(t, \omega^{j}\right)$ to be the conditional probability that exactly $m$ more jumps occur in $[0, t]$, given $\omega^{j}, \tau_{j}<t$. Assume the following inequality

$$
\Theta_{m}\left(t, \omega^{j}\right) \leq \Phi(t, j) v(t, j)^{m-j}
$$

for some positive numbers $\kappa, \Phi(t, j), v(t, j), v(t, j) \in(0,1 /(1+\kappa))$, where $|g(t, x, j)| \leq \alpha+\kappa|x|$ for all $t, x, j, \alpha$ some given positive number, $\kappa$ being also a Lipschitz rank of $x \rightarrow g(t, x, j), j=1,2, \ldots$. Again a stochastic disturbance $V$ can be allowed, with modifications as described in Remark 8 .

The necessary conditions, with $\Lambda_{0}=1$ are sufficient if $x \rightarrow$

$\max _{u \in U}\left\{f_{0}(t, x, u, j) \mu\left((t, \infty) \mid \tau_{0}, \ldots, \tau_{j}\right)+p^{*}(t, \omega) f(t, x, u, j)\right\} D \mu\left(\tau_{0}, \ldots, \tau_{j}\right)$, $x \rightarrow p^{*}(t, \omega) g(t, x, j), x \rightarrow g_{0}(t, x, j)$, and $x \rightarrow h_{0}(x)$ are concave for $t \in$ $\left(\tau_{j}, \tau_{j+1}\right)$.

The theory above applies also to the case where $f$ and $f_{0}$ depend on the $\tau_{j}$ 's, provided, for any given $(x, u)$, these functions are nonanticipating in $(t, \omega)$. In Theorem 1, as well as in [27], [28], in the functions $f_{0}, f, f_{0, x}, f_{x}$ we simply replace $j$ by $\tau_{0}, \ldots, \tau_{j}$. Now, we must assume that the Lipschitz continuity is uniform in $\omega$, (i.e the Lipschitz constants are independent of $\omega$ ), and that inequality [19] holds for $f_{0 x}$ and $f_{x}$ for all $\omega$. Moreover, it is assumed that $f_{0}, f, f_{0 x}$ and $f_{x}$ are separately piecewise and right-continuous in each $\tau_{i}$.

The same solution procedure applies as before: Let us relate it to the necessary conditions [27]-[29], (with $\Lambda_{0}=1$ ):

First, let $\hat{u}\left(t, x, p^{*}, \tau_{0}, \ldots, \tau_{j}\right)$ maximize $f_{0}\left(t, x, u, \tau_{0}, \ldots, \tau_{j}\right) \mu\left((t, \infty) \mid \tau_{0}, \ldots, \tau_{j}\right)+$ $p^{*} f\left(t, x, u, \tau_{0}, \ldots, \tau_{j}\right)$. Then, in the case $N<\infty$, consider the equations

$$
\dot{x}\left(t, \tau_{0}, \ldots, \tau_{j}\right)=f\left(t, x, \hat{u}\left(t, x, p^{*}, \tau_{0}, \ldots, \tau_{j}\right), \tau_{0}, \ldots, \tau_{j}\right)
$$

$$
\dot{p}^{*}\left(t, \tau_{0}, \ldots, \tau_{j}\right)=
$$




$$
\begin{gathered}
-f_{0 x}\left(t, x, \hat{u}\left(t, x, p, \tau_{0}, \ldots, \tau_{j}\right), \tau_{0}, \ldots, \tau_{j}\right) \mu\left((t, \infty) \mid \tau_{0}, \ldots, \tau_{j}\right) \\
-p^{*} f_{x}\left(t, x, \hat{u}\left(t, x, p^{*}, \tau_{1}, \ldots, \tau_{j}\right), \tau_{0}, \ldots, \tau_{j}\right) \\
-g_{0 x}(t, x, j+1) \dot{\mu}\left(t \mid \tau_{0}, \ldots, \tau_{j}\right) \\
-p^{*}\left(t+, \tau_{0}, \ldots, \tau_{j}, t\right)\left[I+g_{x}(t, x, j+1)\right] \dot{\mu}\left(t \mid \tau_{0}, \ldots, \tau_{j}\right) .
\end{gathered}
$$

Boundary conditions for $\left(x\left(t, y, \tau_{0}, \ldots, \tau_{j}\right), p\left(t, y, \tau_{0}, \ldots, \tau_{j}\right)\right)$ are again, for $s=$ $\tau_{j}$, the two equalities $x\left(\tau_{j}\right)=y$, and

$$
p^{*}(T)=h_{0 x}(x(T)) \mu\left((t, \infty) \mid \tau_{0}, \ldots, \tau_{j} .\right)
$$

In case $N<\infty$, again these equations can be solved backwards. First for $j=N$, solutions of the equations are found which we denote $x\left(t ; y, \tau_{0}, \ldots, \tau_{N}\right)$, $p^{*}\left(t ; y, \tau_{0}, \ldots, \tau_{N}\right)$. Next, for $j=N-1$, solutions of the equations are found which we denote $x\left(t ; y, \tau_{0}, \ldots, \tau_{N-1}\right), p^{*}\left(t ; y, \tau_{0}, \ldots, \tau_{N-1}\right)$, (in this case knowledge of $p^{*}\left(t ; y, \tau_{0}, \ldots, \tau_{N-1}, t\right)$ is needed $)$, and so on.

An analogous solution method involving the $p$-functions rather than the $p^{*}$ functions can be written down. For brevity it is dropped.

\section{Problems with terminal constraints}

Let us next consider problems with terminal constraints. The tools available in the general case are a little complicated, so we first consider a very special situation, where $x$ is a scalar, the jump sizes are constant, the scrap value function is zero, and where the terminal inequality condition $x(T-, \omega) \geq \hat{x}$ a.s., is imposed, $\hat{x}$ a given number. Such a terminal condition is sometimes called hard, as opposed to a soft one, that takes the form $\operatorname{Ex}(T-, \omega) \geq \hat{x}, \hat{x}$ a given number. Below, mainly hard end constraints are treated. Soft end constraints are briefly mentioned in Remark 15 below. Thus, we now have a system that, in addition to [9]-[11], includes the conditions:

$$
x \in \mathbb{R}, h_{0} \equiv 0, g \equiv c \neq 0, x(T-, \omega) \geq \hat{x} \text { a.s. }, N<\infty
$$

and that $f_{0}$ and $f$ are independent of $j$. Now, if $j$ jumps have occurred, then as long as no further jumps occur, $x(t ; s, y, j)$ has to be steered in such a manner that if none, or one or more jumps occur in the future (after $t$ ), we are able to satisfy the end constraint. At most $N-j$ jumps can occur after $s$. Even if $t$ is very close to $T$, such a number of jumps can occur with positive probability. If $c>0$, we often need only be concerned with satisfying the 
inequality $x(T-, s, y, j) \geq \hat{x}$ : It this inequality is satisfied, and given any $t$ close to $T$, and also given that $j$ jumps have occurred in $[0, t]$, then if one or more jumps occur after $t$, it is easier to obtain $x\left(T-; s, y, j^{\prime}\right) \geq \hat{x}, j^{\prime}>j$, (sometimes these inequalities will even be automatically satisfied). If $c<0$, a more detailed discussion is useful. We will only consider two situations.

$$
\begin{aligned}
& \sup _{u \in U} f(t, x, u)=+\infty \text { for all }(t, x) \text { in a neighborhood of } \\
& \{(T, x): \hat{x} \leq x \leq \hat{x}-N c\}
\end{aligned}
$$

or, for some constant $K$,

$$
\begin{aligned}
& \sup _{u \in U} f(t, x, u) \leq K \text { for all }(t, x) \text { in a neighborhood of } \\
& \{(T, x): \hat{x} \leq x \leq \hat{x}-N c\}
\end{aligned}
$$

Let $\hat{x}^{j}=\hat{x}$ both in case $c$ is positive, and in case [34], (even if $c$ is negative). In the case where $c$ is negative and [35] holds, let $\hat{x}^{j}=\hat{x}-(N-j) c$. In this case, we have to arrange it so that if $j$ jumps have occurred, then we must make sure that $x(T-; s, y, j)+k c \geq \hat{x}$ for $k=0, \ldots, N-j,(k$ jumps downwards can occur arbitrarily close to $T$. The most demanding equality is obtained for $k=N-j$. So, in general we have to seek solutions $x(t ; s, y, j)$ satisfying the end condition

$$
x(T ; s, y, j) \geq \hat{x}^{j} .
$$

As usual, there is a slackness condition that the adjoint function must satisfy at time $T$. The functions $p(T ; s, y, j)$ must satisfy the transversality conditions

$$
p(T ; s, y, j) \geq 0,=0 \text { if } x(T ; s, y, j)>\hat{x}^{j}, j=0, \ldots, N .
$$

The solution method for problem [9]-[11], [33], is the same as before, except that the boundary conditions are:

$$
x(s ; s, y, j)=y, \quad[36] \text { and }[37]
$$

The solution method now consist of [12]-[14], [38].

Example $3 \max E\left\{\int_{0}^{3}(3-s)(1-u) d s\right\}$, subject to $\dot{x}=u \in[0,1], x(0)=0$, $x(3) \geq 1$ with probability 1 . There is a probability with intensity $\lambda>0$ that a single unit jump downwards occurs.

The conditions [12]-[14], [38] will be applied. After a jump, the solution is $u(t ; s, y, 1)=0$ if $y \geq 1$, and if $y<1, u(t ; s, y, 1)=0$ for $s<y+2$, 
while $u(t ; s, y, 1)=1$ for $t>y+2$. To see this note that $p(t ; s, y, 1)=$ $p(s ; s, y, 1)$. From the maximum condition, evidently $u=0$ to begin with (where $3-t>p$ ), (if at all) and $u=1$ at the end (if at all). Consider the case where we do have a point $\sigma \in(0,1)$ at which we switch from 0 to 1 . Then, $x(t ; s, y, 1)=y$ for $t<\sigma, x(t ; s, y, 1)=y+t-\sigma$ for $t>\sigma$, with $\sigma$ determined by $x(3 ; t, y, 1)=1$, i.e. $y+3-\sigma=1$, or $\sigma=y+2$. At $s=\sigma$, by the maximum condition, $3-\sigma=p(\sigma, s, y, 1)$, so $p(t ; s, y, 1)=1-y,(p$ is independent of $s$ and $t$ ). The formula for $p(t ; s, y, 1)$ is valid if $\sigma \in(s, 3)$, i.e. $y \in(s-2,1)$. It works also for $\sigma=s,(y=s-2)$ and we use it, though then any $p(t ; s, y, 1) \geq 1-y$ would also work. The general formula for $p(t ; s, y, 1)$ is then $p(t ; s, y, 1)=\max (0,1-y), y \geq s-2$, (from points $(s, y), y<s-2$, it is not possible to reach $(3,1)$ (or above).

To find $u(t ; s, y, 0)$, then, again, from the maximum condition, $u=0$ when $3-t>p$ (this happens to begin with, if at all), while $u=1$ when $3-t<p$ (this happens at the end, if at all). For $j=0$ we need only consider $(s, y)=(0,0)$, below $p(t)$ is a shorthand for $p(t ; 0,0,0)$, similarly $x(t)=x(t, 0,0,0)$.

We guess, or try the possibility, that $x(t)-1 \leq 1$, in which case $p(t ; t, x(t)-$ $1,1)=2-x(t)$. Now, $d p / d t=\lambda p(t)-\lambda p(t, t, x-1,1)=\lambda p-\lambda 2+\lambda x(t, 0,0,0)$, while $d x / d t=0$ if $3-t>p$ and $d x / d t=1$ if $3-t<p$. We guess that the two last inequalities occur on intervals $[0, \alpha)$, and $(\alpha, 3]$, respectively, (this will be confirmed). Thus on the first interval, $x(t)=0$, and so $p(t)=2+C e^{\lambda t}$ here, for some integration constant $C$. On the last interval, $x(t)=D+t, D$ an integration constant, and we guess that $x(3)=2$.(We are in case (B) so a jump may occur close to $s=3$, i.e. $x(T-)$ has to equal at least 2$)$. Thus, $D=-1$, which means $\alpha=1$, and which gives that $d p / d t=\lambda p(t)-\lambda 2+\lambda(t-1)$ for $t \in(1,3), d p / d t=\lambda p(t)-\lambda 2$ for $t \in(0,1)$. (One may note that we use the unique formula for $p(t, t, y, 1)$ for $t<1$ in these cases, since $0<1-y=2-x(t)$ for $t<2$.) $y=x(t, 0,0,0)-1 \in(t-2,1)$, for $t<1$.)

This equation has the solution $p(t)=3-t-1 / \lambda+e^{\lambda(t-1)} / \lambda$ for $t \in(1,3)$, (the integration constant is determined by $3-\alpha=p(\alpha)$, i.e. $2=p(1)$ ) and for $t \in(0,1), p(t)=2,(2=p(1)$ gives also $C=0)$.

Let us confirm a couple of properties: The transversality condition required in [25] is satisfied $(p(3)>0)$. Moreover, as $3-t$ is decreasing and $p(t)$ is non-decreasing, the maximum condition is satisfied by $u=0$ before $\alpha=1$, and by $u=1$ afterwards. Note that a solution has to be found such that the end constraint is met with probability 1 . This requires $x(T-, 0,0,0) \geq 2$. The solution $x(t ; 0,0,0)=\max \{0, t-1\}$ satisfies the latter inequality and it describes the optimal behaviour before a jump. After a jump, we use $u=0$ until $t=1$, and then $u=1$, if the jump occurs in [0,1), if the jump occurs in $[1,3)$, we use $u=1$ all the time after the jump. 
Note that the solution is the same for all $\lambda>0$. Sufficient conditions, see Remark 12 below, apply here.

Example 4 Consider the problem

$$
\max E \int_{0}^{T}-u^{2} / 2 d t, \quad \dot{x}=u, x(0)=-2, x(T) \geq 0 \text { a.s., } u \geq 0
$$

with a possibility for a single, unit upwards jump in $x(t)$ at $\tau \in[0, \infty), \tau$ exponentially distributed with intensity $\lambda$. When $y \geq 0$, evidently $u(t ; s, y, 1)=$ $u(t ; s, y, 0)=0$ are optimal, (with the $p$-function vanishing), so we consider only $y$ 's satisfying $y<0$. To find $u(t ; s, y, 1)$, the solution method [12]-[14],[38] is used, and we seek a solution satisfying $x(T ; s, y, 1) \geq 0$. In fact, we guess that, after a jump has occurred, we steer $x$ in such a way that $x(T ; s, y, 1)=0$ if $y \leq 0$. (The guess is confirmed below .) As $u=p(t ; s, y, 1) \equiv \hat{p}(s, y)$, we choose $\hat{p}(s, y)$ such that if we start in $(s, y)$, with $y<0$, we end in $(T, 0)$, i.e., $x(T ; s, y, 1)=y+\int_{s}^{T} \hat{p}(s, y) d t=0$, or $\hat{p}(s, y)=-y /(T-s)$. Moreover, $u(t ; s, y, 1)=y /(s-T), x(t ; s, y, 1)=$ $y(t-T) /(s-T)$ for $y<0$.

To find $u(t ; s, y, 0), y<0$, consider the characteristic equations: $\dot{x}=p$, $\dot{p}=-\lambda p(t ; t, x+1,1)+\lambda p=\lambda(x+1) /(T-t)+\lambda p$. If $x+1 \geq 0$, drop the term $\lambda(x+1) /(T-t))$ in the second equation. Differensiating the first equation and inserting from the second and first equations give $d^{2} x / d t^{2}=$ $\lambda d x / d t-\lambda(x+1) /(t-T)$, for $x \leq-1$, (drop the last term in the opposite case), with boundary conditions $x(s ; s, y, 0)=y, x(T ; s, y, 0)=0$. Defining $w(t)$ by $w(t)(t-T)=x+1$, we get $d x / d t=(d w / d t)(t-T)+w$ and $d^{2} x / d t^{2}=$ $\left(d^{2} w / d t^{2}\right)(t-T)+2 d w / d t=\lambda d x / d t-\lambda(x+1) /(t-T)=\lambda(d w / d t)(t-T)+$ $\lambda w-\lambda w$, so $d^{2} w / d t^{2}=(\lambda-2 /(t-T)) d w / d t$. Integrating this separable equation, we get $\dot{w}(t)=C e^{\lambda t} /(t-T)^{2}$, and $w(t)=D+\int_{s}^{t} C e^{\lambda \sigma} /(\sigma-T)^{2} d \sigma$, so $w(t)(t-T)-1=x(t)=x(t ; s, y, 0)=\beta(t ; s, y):=(t-T)\left[D+\int_{s}^{t} C e^{\lambda \sigma} /(\sigma-\right.$ $\left.T)^{2} d \sigma\right]-1=(T-t)\left[(y+1) /(T-s)-\int_{s}^{t} C e^{\lambda \sigma} /(T-\sigma)^{2} d \sigma\right]-1$, as long as $x(t) \leq$ -1 , (we have used $x(s)=y$ to determine $\mathrm{D}$ ). The equation for $x$ is simpler when $y>-1$, then $x(t ; s, y, 0)=D^{\prime}+C^{\prime} e^{\lambda(t-s)}$. With $x(T)=0, x(s)=y$, this gives $x(t ; s, y, 0)=\beta^{*}(t ; s, y):=-y e^{\lambda T} /\left(e^{\lambda s}-e^{\lambda T}\right)+y e^{\lambda t} /\left(e^{\lambda s}-e^{\lambda T}\right)$, for $y \in(-1,0)$.

Ultimately, before any jump, we only need to know the solution $x(t ; s, y, 0)$ for $(s, y)=(0,-2)$. The solution $x(t ; 0,-2,0)$ is "smoothly pasted" together at the point $t^{\prime \prime}<T$ at which the line $x=-1$ is crossed, by putting $-1=$ $\beta\left(t^{\prime \prime}, 0,-2\right)$, as well as by putting the first derivatives equal to zero, which yields $\lambda e^{\lambda t^{\prime \prime}} /\left(e^{\lambda T}-e^{\lambda t^{\prime \prime}}\right)=\left[\partial \beta^{*}\left(t ; t^{\prime \prime},-1\right) / \partial t\right]_{t=t^{\prime \prime}}=[\partial \beta(t ; 0,-2) / \partial t]_{t=t^{\prime \prime}}=$ $(-1)\left[-1 / T-\int_{0}^{t^{\prime \prime}} C e^{\lambda \sigma} /(\sigma-T)^{2} d \sigma\right]-C e^{\lambda t^{\prime \prime}} /\left(T-t^{\prime \prime}\right)$. (The first characteristic 
equation does tell that $\dot{x}$ is continuous.) The two equations determine $C$ and $t^{\prime \prime}$ : The first equation reduces to $-1 / T-C \int_{s}^{t^{\prime \prime}} e^{\lambda \sigma} /(T-\sigma)^{2} d \sigma=0$, using this in the second one, it reduces to $\lambda e^{\lambda t^{\prime \prime}} /\left(e^{\lambda T}-e^{\lambda t^{\prime \prime}}\right)=$ $-C e^{\lambda t^{\prime \prime}} /\left(T-t^{\prime \prime}\right)$, or $\lambda /\left(e^{\lambda T}-e^{\lambda t^{\prime \prime}}\right)=-C /\left(T-t^{\prime \prime}\right)$, which gives $C=\left(t^{\prime \prime}-\right.$ $T) \lambda /\left(e^{\lambda T}-e^{\lambda t^{\prime \prime}}\right)$. Using this, the first equality becomes $-1 / T=-\left\{\lambda\left(T-t^{\prime \prime}\right) /\left(e^{\lambda T}-e^{\lambda t^{\prime \prime}}\right)\right\} \int_{0}^{t^{\prime \prime}} e^{\lambda \sigma} /(T-\sigma)^{2} d \sigma$.

For $t^{\prime \prime}=0$, the right-hand side is 0 , while for $t^{\prime \prime}=T$ it is $-\infty$, (when $t^{\prime \prime} \rightarrow T$, the square bracket has a positive limit, while the integral diverges). So a solution for $t^{\prime \prime}$ and hence also for $C$ exist.

Having found $C$ and $t^{\prime \prime}$, if $y<-1$, then $x(t ; 0,-2,0)=\beta(t ; 0,-2)$ for $t$ in $\left(0, t^{\prime \prime}\right)$ and $x(t ; 0,-2,0)=\beta^{*}\left(t ; t^{\prime \prime},-1\right)$ for $t \geq t^{\prime \prime}$. Using sufficient conditions based on concavity (Remark 12 below), it can be shown that the solutions $x(t ; s, y, 1)$ and $x(t ; 0,-2,0)$ are optimal. Here the solution depends on $\lambda$.

\section{The general end constrained case}

The following ("hard") end conditions are imposed. With probability 1,

$$
\begin{gathered}
x_{i}(T-, \omega)=\hat{x}_{i}, \quad i=1, \ldots, n^{\prime} \\
x_{i}(T-, \omega) \geq \hat{x}_{i}, \quad i \geq n^{\prime}+1, \ldots, n^{\prime \prime}
\end{gathered}
$$

In addition to the earlier assumptions on the system mentioned subsequent to Theorem 1, it is now assumed that $f_{0 x}$ and $f_{x}$ are uniformly continuous in $(x, u)$, uniformly in $(t, j)$, and that $g_{x}(t, x, j)$ is uniformly continuous in $x$, uniformly in $t, j$, that, for all $t, x,\left|g_{x}(t, x, j)\right| \leq M_{j}^{\prime},|g(t, 0, j)| \leq M_{j}^{\prime}$, for some constants $M_{j}^{\prime}$, and that

$$
\sum_{j=1}^{\infty} M_{j}^{\prime}<\infty
$$

Let $\Pi$ be the map $\left(x_{1}, \ldots, x_{n}\right) \rightarrow\left(x_{1}, \ldots, x_{n^{\prime \prime}}\right)$, and let

$$
\hat{B}=\left\{x \in \mathbb{R}^{n}: x_{i}=0, i \leq n^{\prime}, x_{i} \geq 0, n^{\prime}+1 \leq i \leq n^{\prime \prime}\right\} .
$$

The possibility of being able to satisfy [39] depends on the behaviour of the process (i.e. on the "controllability" properties of the process). For example, if $f$ is a bounded function (case [42 b] below), we cannot hope to have [39a]) satisfied, when large jumps in $x_{i}(),. i \leq n^{\prime}$, occur close to $T$. Define $g^{0, j}(x)=x, g^{1, j}(x)=x+g(T, x, j+1), g^{2, j}(x)=g^{1, j}(x)+g\left(T, g^{1, j}(x), j+2\right)$, and recursively $g^{k, j}(x)=g^{k-1, j}(x)+g\left(T, g^{k-1, j}(x), j+k\right)$. We shall only discuss systems satisfying one of the two condition: For some $T^{\prime} \in[0, T)$, 


$$
\begin{aligned}
& \mathbb{R}^{n^{\prime \prime}} \subset \Pi\left\{f\left(t, x^{*}\left(t, \omega^{j}\right), u, j\right)-b: u \in U, b \in \hat{B}\right\} \\
& \quad \text { for all }(t, \omega), t \in\left[T^{\prime}, T\right]
\end{aligned}
$$

or

$$
\sup _{j, t \in\left[T^{\prime}, T\right], u \in U}\left|\Pi f\left(t, x^{*}\left(t, \omega^{j}\right), u, j\right)\right|<\infty \text { for all } \omega, g_{i} \equiv 0, i \leq n^{\prime}
$$

We shall now require that for a pair $x(t, \omega), u(t, \omega)$ to be called admissible, the inequalities $\sup _{t, \omega}|x(t, \omega)|<\infty$ and, (for any $T^{\prime}<T$ ), $\sup _{t \leq T^{\prime}, \omega}|u(t, \omega)|<$ $\infty$ have to be satisfied. (Note the occurrence of $T^{\prime}<T$ in the last inequality, Example 4 explains why.)

In necessary conditions, $x^{*}(t, \omega)$ is assumed to be the optimal solution. But, as for other constraints qualifications, in practice, we need that [42] holds for all admissible pairs. (If [42] fails for some admissible solutions, it must automatically be considered a candidate for optimality.) The problem now consists of [9]-[11], [39] ],[40], [42]. For solutions to be admissible, [39 ] has to hold. As long as only $j$ jumps have occurred, in case [42b], we have to steer the system in such a manner that the equalities $x_{i}=\hat{x}_{i}, i \leq n^{\prime}$ and the inequalities $\left(g^{k, j}(x)\right)_{i} \geq \hat{x}_{i}, k=0, \ldots, N-j, i=n^{\prime}+1, \ldots, n^{\prime \prime}$ hold for $x=x(T-; s, y, j)$. The reason is that, however close $t$ is to $T$, further jumps can occur. In case [42 a], we steer the system such that [39] holds.

\section{Solution method in the end constrained case, with $N<1$}

Assuming that $f, f_{0}, g_{0}, g$, and $h_{0}$ are $C^{2}$, the method for finding solutions described in connection with [12]-[14] ([4]-[6] above also works here, the only change needed is a change of the terminal condition on $p(T)$ : We will need numbers, (multipliers), $\Lambda_{i}(k, s, y, j), k=0, \ldots, N-j, i=1, \ldots, n^{\prime \prime}$, (with $\Lambda(k, s, y, j):=\left(\Lambda_{1}(k, s, y, j), \ldots, \Lambda_{n^{\prime \prime}}(k, s, y, j), 0, \ldots, 0\right) \in \mathbb{R}^{n}$, to formulate the condition.

$$
\begin{gathered}
p(T ; s, y, j)=\left(h_{0 x}(x(T ; s, y, j))+\sum_{0 \leq k \leq N-j} \Lambda(k, s, y, j) g_{x}^{k, j}(x(T ; s, y, j)),\right. \\
\Lambda_{i}(k, s, y, j) \geq 0, \Lambda_{i}(k, s, y, j)=0 \text { if }\left(g^{k, j}(x(T ; s, y, j))\right)_{i}>\hat{x}_{i}, i=n^{\prime}+1, \ldots, n^{\prime \prime} . \\
p_{i}(T ; s, y, j)=\left(h_{0 x}(x(T ; s, y, j))_{i}, i>n^{\prime \prime} .\right. \\
\Lambda_{i}(k, s, y, j)=0, i \leq n^{\prime}, k>0, \quad \text { and also for } i>n^{\prime} \text { in case }[42 a],
\end{gathered}
$$

To solve the end constrained problem, (i.e. to find characteristic pairs) one applies [12]-[14], with boundary conditions

$$
\text { [39] ],[43] and } x(s ; s, y, j)=y
$$


(Formally, there may be a need to find also "abnormal" triples, this is commented upon subsequent to Theorem 4 below.)

Remark 2 describes how characteristic triples $x(t ; s, y, j), u(t ; s, y, j), p(t ; s, y, j)$ give rise to corresponding non-anticipating entities $x(t, \omega), u(t, \omega), p(t, \omega)$. Similarly, to $\Lambda(k, s, y, j)$ corresponds $\Lambda\left(k, \tau_{1}, \ldots, \tau_{j}\right):=\Lambda\left(k, \tau_{j}, x\left(\tau_{j}+; \tau_{1}, \ldots, \tau_{j}\right), j\right)$, when $\tau_{j}<T$. (We can even write $\Lambda(k, \omega)$ instead of $\Lambda\left(k, \tau_{1}, \ldots, \tau_{j}\right), j=$ $0,1 \ldots, N$, if we agree that $\Lambda(k, \omega)$ is a function that is non-anticipating at time $T$.)

\section{Remark 11 Which start-points should enter characteristic triples}

Let us discuss a little more the start points $(s, y)$ used to construct the characteristic solutions.

At the outset, we must assume that admissible solutions exist. Now admissibility implies that the terminal conditions are satisfied, and this sense of admissibility should also be understood when reading the definition of $Q(j)$ in [20]. The just mentioned assumption implies that $Q(j)$ is nonempty. As before, solutions $x(t ; s, y, j)$ must be constructed for all points in $Q(j)$, (as well as for $\left(0, x^{0}\right)$ when $\left.j=0\right)$.

In a sufficient condition below larger open sets $Q^{0}(j)$ appear. Sometimes they can be constructed again by considering all solutions $x(t, \omega)$, starting at $t=0$ at all $\hat{x}^{0}$ in an open ball around $x^{0}$ as mentioned in the discussion subsequent to [20]. Occasionally, even $Q(j)=(0, T) \times \mathbb{R}^{n}$ works. But note that now it can be more difficult (or in some cases impossible) to find such larger open sets $Q^{0}(j)$, that have the property that characteristic solutions can start anywhere in these sets and still satisfy the terminal conditions.

To obtain a maximum principle (necessary condition), a condition is needed that secures that "enough choices" of values of $\dot{x}$ are available: Let $\Pi$ be the map $\left(x_{1}, \ldots, x_{n}\right) \rightarrow\left(x_{1}, \ldots, x_{n}\right)$. For some $\eta>0, c>0$, some bounded non-anticipating function $z(t, \omega)$, piecewise and left-continuous in $t$, rightcontinuous in each $\tau_{i}$, for all $\omega$, for all $t \in[T-\eta, T]$ such that $t \in\left(\tau_{j}, \tau_{j+1}\right)$,

$$
B\left(\dot{x}^{*}(t, \omega)+z(t, \omega), \eta\right) \subset \Pi\left\{f\left(t, x^{*}(t, \omega), u, j\right)-b: u \in U \cap B(0, c), b \in \hat{B}\right\}
$$

In case of [42 a], this condition often follows automatically.

The following maximum principle will normally hold as a necessary condition for optimality. The assumptions on $f_{0}, f, g_{0}, g, h_{0}$ subsequent to [39] and 
to Theorem 1 are again postulated. Moreover, it is assumed that, for some $\hat{d}>0, \Pi f_{x}\left(t, x, u^{*}(t, \omega), j\right)$ is uniformly continuous in $x \in B\left(x^{*}(t, \omega), \hat{d}\right)$, uniformly in $t, \omega, j$. (Alternative assumptions are stated in Remark 18.)

\section{Theorem 4 (Necessary conditions, end constrained case)}

Let $x^{*}(t, \omega), u^{*}(t, \omega)$ be an optimal pair in the end constrained problem [9][11], [39] ],[40], [42]. Assume [45]. Then Theorem 1 holds with two modification: The transversality condition [18] on $p(T, \omega)$ is changed to the following one: There exist multipliers $\Lambda_{i}\left(k, \tau_{1}, \ldots, \tau_{j}\right), k=0, \ldots, N-j, i=1, \ldots, n^{\prime \prime}$ such that [43] holds for $\left(\Lambda_{i}(k, s, y, j), p(T ; s, y, j), x(T ; s, y, j), h_{0}(x(T ;, s, y, j))\right.$ replaced by $\left(\Lambda_{i}\left(k, \tau_{1}, \ldots, \tau_{j}\right), p\left(T ; \tau_{1}, . ., \tau_{j}\right), x^{*}\left(T ; \tau_{1}, \ldots, \tau_{j}\right), \Lambda_{0} h_{0}\left(x^{*}\left(T ; \tau_{1}, \ldots, \tau_{j}\right)\right)\right.$. Moreover, in [16]-[17], $f_{0}, f_{0 x}$, and $g_{0 x}$ are replaced by $\Lambda_{0} f_{0}, \Lambda_{0} f_{0 x}$, and $\Lambda_{0} g_{0 x}$, respectively. Here, $\Lambda_{0} \in\{0,1\}$. Finally,

$$
\sup _{|z(t, \omega)|<1} E \int_{0}^{T} p(t, \omega) z(t, \omega) d t<\infty,
$$

$(z(t, \omega)$ having properties as in [45]).

The functions $\Lambda\left(k, \tau_{1}, \ldots, \tau_{j}\right)$ can normally be assumed to be bounded functions, and piecewise and right-continuous in each variable separately. The condition [45] will automatically ensure $\Lambda_{0}=1$ if $z(t, \omega)=0$.

Unless sufficient conditions, (Theorems 5, 6 below) are used, to ensure that all candidates for optimality have been found, it is necessary to apply the solution procedure both for $\Lambda_{0}=1$ and $\Lambda_{0}=0$, (the latter value requires obvious modifications of [12]-[14], [43], compare the ones occurring in Theorem 4.)

The word "normally" was used a couple of times above to indicate that there are cases, rather exceptional ones, in which necessary conditions require multipliers less well-behaved than the above ones. A proof of such conditions appears in Seierstad (2002).

The sufficient conditions in Theorem 2 now takes the following form, (formally we then need not assume [45]):

\section{Theorem 5 (Sufficient conditions, end constrained problems)}

If we substitute the transversality condition of Theorem 1 with the one in 
Theorem 4 for $\Lambda_{0}=1$, then Theorem 2 still holds in the end constrained case.

\section{Remark 12 (Sufficient concavity condition for characteristic triples)}

Similarly, the characteristic triples $x(t ; s, y, j), u(t ; s, y, j), p(t ; s, y, j)$ yield an optimal control, if the concavity conditions in Remark 6 holds.

In the next sufficiency theorem, we need a new boundary condition. To formulate it, a few entities need to be defined. Define $s \rightarrow z(t ; s, y, j)$ to be the solution of the following differential equation (linear in $z$ ), when $(x(t ; s, y, j), p(t ; s, y, j))$ is inserted for $(x, p)$ :

$$
\begin{aligned}
& \dot{z}(t)=\lambda(j+1)\left\{z-g_{0}(t, x, j+1)-z(t, t, x+g(t, x, j+1), j+1)\right\}- \\
& f_{0}(t, x, \hat{u}(t, x, p, j), j), \quad z(T)=h_{0}(x(T ; s, y, j))
\end{aligned}
$$

(It is the same as [23] above.) Assume that all $x(t ; s, y, j), p(t ; s, y, j)$ are known. Solving [47] by using backwards recursion yields solutions $z(t ; s, y, j)$. (With $\lambda(N+1)=0, z(t ; s, y, N)$ can first be constructed from [47].) Define

$$
J(t, x, j):=z(t ; t, x, j) .
$$

The boundary conditions are: For any admissible solution $\hat{x}(t, \omega)$,

$$
\begin{aligned}
& \sup _{t, \omega \in\left\{\omega: \tau_{j}<T \leq \tau_{j+1}\right\}} \mid J\left(t, \hat{x}(t, \omega), j \mid<\infty, \text { and for all } \omega \in\left\{\omega: \tau_{j}<T \leq \tau_{j+1}\right\}\right. \\
& \lim _{t \rightarrow T} J(t, \hat{x}(t-; \omega), j)=h_{0}(\hat{x}(T-, \omega))
\end{aligned}
$$

The inequality [49] automatically holds in case [22] holds. The boundedness property [22] may however fail to hold in end constrained problems. In such cases [49] is needed.

Theorem 6 (Sufficient conditions for a field of characteristic triples, with $N<\infty$ and terminal constraints) In Theorem 3, make the following changes: Delete [22], add [49], and replace the boundedness properties of $p(s ; s, y, j)$ and $p_{y}(s ; s, y)$ by the conditions that $p(\rho ; \rho, x(\rho ; s, y)+$ $g(\rho, x(\rho ; s, y), j), j+1)$ and $p_{y}(\rho ; \rho, x(\rho ; s, y)+g(\rho, x(\rho ; s, y), j), j+1)$ are locally bounded in $(s, y)$, uniformly in $\rho$. Provided the sets $Q(j)$ are as described in Remark 11, and the characteristic pairs now satisfy the present terminal and transversality conditions [29], [43],[49], with $\Lambda_{i}(k, s, y, j)$ continuous for $i=n^{\prime}+1, \ldots, n^{\prime \prime}$, then the conclusions of Theorem 3 are still valid 
in the end constrained case [9]-[11], [39].

\section{Example 4 revisited}

Theorem 6 can also be applied to obtain optimality of the proposed candidates. Below only condition [49] is discussed: Let $j=1$, and note that, for $y<0, J(s, y, 1):=\int_{s}^{T}-(y /(T-t))^{2} / 2 d t=-y^{2} / 2(T-s)$, whereas $J(s, y, 1)=0$ for $y>0$, so $J(s, y, 1)=-(\min \{0, y\})^{2} / 2(T-s)$. For any $(s, y, 1)$-admissible function $\hat{x}(t)$, with corresponding control $u(t)$, for which $\hat{x}(T)=0, \lim _{t \rightarrow T}-(\min \{0, \hat{x}(t)\})^{2} /(T-t)=0$, because controls are bounded functions, (they have individual bounds). Then [49] holds for $(s, y, 1)$ - admissible solutions that satisfy $\hat{x}(T)=0$. If $\hat{x}(T)>0, \hat{x}(t)>$ 0 for $t$ close to $T$, and for such $t, 0=J(s, \hat{x}(s), 1)=z(s ; s, \hat{x}(s), 1)=$ $\lim _{s \rightarrow T} J(s, \hat{x}(s), 1)$, so [49] also holds in this case. Next, consider the case $j=0$. We need an expression for $J(s, y, 0)$ only for $y \in(-1, \infty)$. Now, $\dot{x}(t ; s, y, 0)=-\lambda \min \{0, y\} e^{\lambda t} /\left(e^{\lambda s}-e^{\lambda T}\right)$, for $y \in(-1, \infty)$, and $J(s, y, 0)=$ $z(s ; s, y, 0)$, where $z(t ; s, y, 0)$ is the solution of $\dot{z}=$

$\lambda z-\lambda(\min \{0, x+1\})^{2} / 2(T-t)-\lambda^{2}(\min \{0, y\})^{2} e^{2 \lambda t} / 2\left(e^{\lambda s}-e^{\lambda T}\right)^{2}=$ $\lambda z-\lambda^{2}(\min \{0, y\})^{2} e^{2 \lambda t} / 2\left(e^{\lambda s}-e^{\lambda T}\right)^{2}$.

Thus, $z(t ; s, y, 0)=-\lambda(\min \{0, x\})^{2}\left(e^{\lambda(t+T)}-e^{2 \lambda t}\right) / 2\left(e^{\lambda s}-e^{\lambda T}\right)^{2}$ and $J(s, y, 0)=$ $-\lambda(\min \{0, y\}) 2\left(e^{\lambda(s+T)}-e^{2 \lambda s}\right) / 2\left(e^{\lambda s}-e^{\lambda T}\right)^{2}=\lambda(\min \{0, y\})^{2} e^{\lambda s} / 2\left(e^{\lambda s}-e^{\lambda T}\right)$ for $y \in(-1, \infty)$. Having this formula, a test of [49], as carried out above, can again be done for $j=0$. The case $\hat{x}(T)>0$ is trivial. If $\hat{x}(T)=0$, $z(s ; s, \hat{x}(s), 0)=J(s, \hat{x}(s), 0)=\lambda(\min \{0, \hat{x}(s)\})^{2} e^{\lambda s} / 2\left(e^{\lambda s}-e^{\lambda T}\right) \rightarrow 0$, when $s \rightarrow T$, because controls are bounded.

Sometimes, Theorem 6 does not apply, because solutions with open domains $Q(j)$ for $(s, y)$ do not exist. Consider the following example:

\section{Example 5}

$$
\max E \int_{0}^{3} u t d t, \quad \dot{x}=-u, \quad u \in[0,1], x(0)=1, x(3-) \geq 0 \text { a.s. },
$$

with a possibility for a single, unit upwards jump in $x(t)$, with probability given by the intensity $\lambda$.

a. Considering first the situation after a jump with initial condition $(s, y)$. Let us intuitively construct the solution in this case. To get some "sizable" positive value of the criterion, we have to use $u=1$ for some time, The constraint $x(3) \geq 0$ limits the time interval on which we can use $u=1$. It evidently pays to postpone the use of $u=1$ as long as possible, due to the factor $t$ in the integrand. Hence, the optimal path is evidently $x=y$ until 
time $t^{\prime \prime}$ given by $y=3-t^{\prime \prime}$. Afterwards, $u=1$ and this yields $x(3)=0$. Here we assumed $s<3-y$. Now, $p(t ; s, y, 1)$ is constant, and to have a switch point at $t^{\prime \prime}, p\left(t^{\prime \prime} ; s, y\right)=3-y$. If on the other hand $s>3-y$, we can use $u=1$ all the time, and still we do not reach $(3,0)$, so $0=p(3 ; s, y, 1)=p(t ; s, y, 1)$. If $s=3-y, u=1$ all the time, in this case any $p(t ; s, y, 1) \equiv p^{*} \in[0, s]$ works. Evidently, $x(t, s, y, 1)=\max \{y, \max \{s, 3-y\}+y-t\}=$ $y+\max \{0, \max \{s, 3-y\}-t\}$.

b. Before a jump, we need only consider the case $(\mathrm{s}, \mathrm{y})=(0,1)$. Our intuition tells us that an optimal policy is as follows. The factor $\mathrm{t}$ in the integrand in the criterion makes it optimal to postpone using $u=1$. When we surely have no jump, $u=1$ is optimal on a unit interval $I$ at the very end. However, with a positive probability for a jump, it may pay to have $I=(a, a+1)$ at which $u=1$ somewhat earlier than at the very end. It may be advantageous to make room for having $u=1$ on an additional set in case of a jump (a jump makes this possible). At least for $\lambda$ small, presumably we will not exploit the latter possibility fully (or at all), for $\lambda$ large, we would expect $a$ to be close to (or equal to) 1 . It can never pay to have $a<1$, for if we experience a jump at any point, $x$ is $\leq 2$ here, and we don't need a larger interval than one of length 2 for $x$ to decrease down to zero.

c. Now, we put the solution method [4]-[6], [38] to work. For adjoint functions $p(t):=p(t ; 0,1,0)$ and $p(t ; s, y, 1), t>\tau$, corresponding to the situation before a jump has occurred, and after a jump has occurred, respectively, the following maximum conditions hold:

$$
(u-u(t ; s, y, 1)) t+p(t ; s, y, 1)(u(t ; s, y, 1)-u) \leq 0 \text { for all } u \in[0,1]
$$

and for $u^{*}(t):=u(t ; 0,1,0)$,

$$
\left(u-u^{*}(t)\right) t+p(t)\left(u^{*}(t)-u\right) \leq 0 \text { for all } u \in[0,1]
$$

where $p(t)$ satisfies the differential equation:

$$
\dot{p}(t)=-\lambda p(t ; t)+\lambda p(t)
$$

$p(t ; t):=p(t ; s, x(t+), 1)$, with end condition $p(3) \geq 0,=0$ if $x^{*}(3-)>0$, (we write $x(t ; 0,1,0)=x^{*}(t)$ ), and where $p(t ; s, y, 1)$, for $t>\tau$ satisfies the adjoint equation $\dot{p}=0, p(3-; s, y, 1) \geq 0,(=0$ if $x(3-; s, y, 1)>0)$.

d. We now take for granted that $u^{*}(T)=0$, for $t \in[0, a], a \geq=1$, $u^{*}(t)=1$ in $(a, a+1)$ and $u^{*}(t)=0$ for $t>a+1$. Observe that if a jump 
occurs at $s \in(1,3]$, then we jump to a point $(s, y)$ satisfying $s \geq 3-y$, with $s>3-y$ if $a>1$. (To see this, note that if $s \leq b:=a+1, x^{*}(s)=1-(s-a)$, so $y=2-(s-a) \geq 2-s+1=3-s$. If $s \geq b \geq 2, x^{*}(s) \geq x(3) \geq 0$, so $y=x^{*}(s)+1 \geq 1$, and $s \geq 2 \geq 3-y$, in fact, $s \geq b>2$, if $a>1$.) From a., recall that $p(t ; s, y, 1)=3-y$, if $s<3-y$, whereas $p(t ; s, y, 1)=0$ for $s>3-y$. Thus, for $s>1$, we jump to a point $(s, y)$ for which $p(s ; s, y, 0)=0$.

e. We shall first consider a "medium sized" value of $\lambda$ : We shall assume that $1 /\left(e^{\lambda}-1\right) \in(1,2)$. Define $h^{*}(t):=\int_{t}^{3} \lambda e^{-\lambda \tau} p(s ; s, x(s ; 0,1,0)+1,1) d \tau$. Evidently, $p(t)=e^{\lambda t}\left(h^{*}(t)+h\right)$ for some constant $h \geq 0$. Assume for the moment that there exists an interval $[a, b] \subset(0,3), b=a+1$, in the interior of which $\psi(t)-h>0, \psi(t):=e^{-\lambda t} t-h^{*}(t)>0$, with the opposite (strict) inequality holding outside $[a, b]$. Then, by $(* *)$, for $t<\tau, u^{*}(t)=1_{(a, b)}$ (the indicator function of the set $(a, b)$ ). If no jump occurs in $[0,3]$, we have guessed $x^{*}(3-)=0$, which is consistent with the above choice of control. (There is a positive probability that there is no jump in $[0,3]$, so we must have $x^{*}(3-) \geq 0$, in the case of no jump in $[0,3]$.). We have also guessed (or we know) that $a \geq 1$, in fact we now guess that $a>1$. Consider first $t \geq a$. Then $h^{*}(t)=0$, (if we jump at $s \geq t$, we jump to a point where $p(t ; s, x(s ; 1,0,0)+1,1)=0$, see d.) Thus, for $t \geq a, \psi(t)=e^{-\lambda t} t=: \phi(t)$. Now, as $a$ and $b$ are switch points, $\psi(a)-h=\psi(b)-h=0$, so $\phi(a)=\phi(b)$, which implies $\alpha(a):=(a+1) e^{-\lambda(a+1)}-a e^{-\lambda a}=e^{-\lambda a}\left(a\left(e^{-\lambda}-1\right)+e^{-\lambda}\right)=0$. This equation always has a solution $a=1 /\left(e^{\lambda}-1\right) \in(0, \infty)$. We have in fact assumed that $1 /\left(e^{\lambda}-1\right) \in(1,2)$, so we have identified the interval $(a, a+1)$ in this case. Note that the function $t e^{-\lambda t}$ increases until $t=1 / \lambda$ and then decreases. Since $b e^{-\lambda b}=a e^{-\lambda a}, 1 / \lambda$ belongs to $(a, b)$ and $t e^{-\lambda t}>a e^{-\lambda a}$ in this interval. Let us show that $u=1$ yields maximum of the Hamiltonian for $t \in(a, b)$, (cf. $\left.\left({ }^{* *}\right)\right)$. A jump after $t$ at $\tau$, gives $p(s ; s)=0$, and $\psi(t)=\phi(t)$, so $\psi(t)-h \geq \phi(a)-h=0$. Consider next $t<a$. Then, $\psi(t) \leq \phi(t) \leq \phi(a)$, so $\psi(t)-h$ is $\leq 0$ showing that $(* *)$ is satisfied for $u^{*}(t)=0$. Similarly, for $t>b=a+1$, $\psi(t) \leq \phi(t) \leq \phi(b)$, so $\psi(t)-h \leq 0$, thus $u^{*}(t)=0$ satisfies $(* *)$. Having made a number of guesses it is reassuring to know that in this problem, sufficient conditions work.

f. For the persisting reader, we have considered other possible cases. Consider first the case where $1 /\left(e^{\lambda}-1\right) \geq 2$. Recall that in the interval $\left(1 /\left(e^{\lambda}-1\right), 1+1 /\left(e^{\lambda-1)} \phi(t):=t e^{-\lambda t}\right.\right.$ first increases until some point and then decreases, with $\phi\left(1 /\left(e^{\lambda}-1\right)\right)=\phi\left(1+1 /\left(e^{\lambda}-1\right)\right)$. Now, we let $a=2, b=3$, 
$h:=\phi(a) \leq \phi\left(1 /\left(e^{\lambda}-1\right)\right)=\phi\left(1+1 /\left(e^{\lambda}-1\right)\right)$. Note that $\psi \leq \phi$ everywhere, in fact $\psi=\phi$ for $t>1$, as $h^{*}(t)=0$ for such $t$. Evidently $\phi(t)=\psi(t)>h$ in $\left(2,1+1 /\left(e^{-\lambda}-1\right)\right)$, which contains $(2,3)$. So for $t \in(2,3)$, by $(* *), u^{*}(t)=1$, $u^{*}(t)=0$ elsewhere, $(\psi(t) \leq \phi(t) \leq \phi(2)$ for $t<2$, since $\psi$ is increasing for $t<2$.)

Finally, consider the case $1 /\left(e^{\lambda}-1\right) \leq 1$, in which case we believe that $a=1$. Note that if $s=3-y$, then $u(t ; s, y, 1) \equiv 1$ and the function $p(t ; s, y, 1)=p(s, y, 1)$ is undetermined, it can take any value in $[0, s]$. A jump at $s>2$, or at $s<1$ brings us to a point $y=x^{*}(s-)+1$ for which $p(t ; s, y, 1)=0$, while if a jump $s$ occurs in $[1,2]$, then we jump to $y=3-s$, $\left(x^{*}(s)=1-(s-1)\right.$, so $\left.y=1+x(s)=2-(s-1)=3-s\right)$, in which case $p(t ; s, y, 1)$ is undetermined in $[0, t]$. Surprisingly, in what follows, we need to specify suitable nonzero values of $p^{s}=p(s ; s, x(s-, 0,1,0)+1,1)$ in $[1,2]$ : and we shall put $p^{s}=\gamma^{*} s, \gamma^{*}$ to be determined. If a jump $s$ occurs before 1 , as we saw above, we jump to $y$ satisfying $s<3-y$, in which case $p^{s}=3-y=1$. Trusting our guess that $u^{*}=1$ in $(a, b)=(1,2)$, (=0 outside), we need to have $\psi(1)=\psi(2)$. This can be obtained if we choose $\gamma^{*} \in[0,1]$ in the following manner: Note that for $t \geq 1, \psi(t)=$ $\phi(t)-\int_{[t, 2]} \lambda e^{-\lambda z} \gamma^{*} z d z=\phi(t)-\left\{\gamma^{*} t e^{-\lambda t}-\gamma^{*} 2 e^{-\lambda 2}+\gamma^{*} e^{-\lambda t} / \lambda-\gamma^{*} e^{-2 \lambda} / \lambda\right\}$. Solve $\psi(2)-\psi(1)=\left(2 e^{-\lambda 2}-e^{-\lambda}\right)\left(1-\gamma^{*}\right)-\gamma^{*}\left(e^{-\lambda 2}-e^{-\lambda}\right) / \lambda=0$ for $\gamma^{*}$, which yields $0 \leq \gamma^{*}=\left(2 e^{-\lambda 2}-e^{-\lambda}\right) /\left[2 e^{-\lambda 2}-e^{-\lambda}+\left(e^{-\lambda 2}-e^{-\lambda}\right) / \lambda\right]<1$. Note that $\psi^{\prime}(t)=e^{-\lambda t}\left(1+\lambda\left(\gamma^{*}-1\right) t\right)$. Now, due to the linear factor in $\psi^{\prime}, \psi^{\prime}$ changes sign at most once in $[1,2]$. In fact, $\psi^{\prime}$ changes sign exactly ones, at a point in $(1,2)$, because $\psi(1)=\psi(2)$. So $\psi(t)$ first increasing and then decreasing in $[1,2]$. Thus, $\psi(t) \geq \psi(b)=: h$, for $t \in(1,2)$. Moreover, $\psi^{\prime}(t)=\phi^{\prime}(t), t>2$, and $\psi(2)=\phi(2)$, with $\phi^{\prime}(t)<0$, for $t>2>1 \geq 1 /\left(e^{\lambda}-1\right)$, so $\psi(t)<\psi(2)$, for $t>2$, and $u^{*}=0$ for such $t$, (compatible with the guess we have already made). Finally, for $t<1, \psi^{\prime}(t)=(d / d t)\left[t e^{-\lambda t}-\int_{t}^{1} \lambda e^{-\lambda \sigma} d \sigma+\right.$ constant $]=$ $e^{-\lambda t}(1-\lambda t)+\lambda e^{-\lambda t}>e^{-\lambda t}>0$. Thus, $\psi(t)<\psi(1)<h$ for $t<1$, which implies $u^{*}=0$ for such $t$ in [**], (compatible with the guess we have already made).

Generally, the results in the various cases above conform with our intuition. In each case, it is easily seen that the candidate proposed satisfies sufficient conditions, (concavity holds).

g. Replace the initial point $(0,1)$ by $\left(0, x^{\prime \prime}\right), x^{\prime \prime} \in(0,2)$, and assume that $2>1 /\left(e^{\lambda}-1\right)>1$. Let us indicate why the optimal value function 
$x^{\prime \prime} \rightarrow J\left(0, x^{\prime \prime}, 0\right)$ is not differentiable: For such an initial condition, the solution is as follows: Before a jump, $u^{*}(t)=1_{\left(a, a+x^{\prime \prime}\right)}$, where $a=1 /\left(e^{\lambda}-1\right)$ if $1 /\left(e^{\lambda}-1\right) \in\left(2-x^{\prime \prime}, 3-x^{\prime \prime}\right)$, with $a$ equal to the left end or right end of this interval in cases analogous to the ones above. Define $\hat{x}^{\prime \prime}$ by $2-\hat{x}^{\prime \prime}=1 /\left(e^{\lambda}-1\right)$. For $x^{\prime \prime}>\hat{x}^{\prime \prime}, a=1 /\left(e^{\lambda}-1\right)$, so $a$ is independent of $x^{\prime \prime}$, whereas if $x^{\prime \prime}<\hat{x}^{\prime \prime}$, then $a$ depends on $x^{\prime \prime}$, in fact $a=2-x^{\prime \prime}$. This entails that $J\left(0, x^{\prime \prime}, 0\right)$ is not differentiable at $x^{\prime \prime}=\hat{x}^{\prime \prime}$.

\section{Remark 13 Stochastic jump sizes}

Even in the end constrained case, the function $g_{0}$ and $g$ can contain disturbances $V$. We confine ourselves to a brief comment on the change needed for the solution procedure connected with [12]-[14] to work in this case, and now it is assumed that the $V_{j}$ 's are stochastically independent of $\tau_{i}, V_{i}, i<j$. The only change needed is a redefinition of $g^{k, j}, k \geq 1$ : Let $\hat{g}^{0, j}(x)=x$, $\hat{g}^{1, j}\left(x, v_{j}\right)=x+g\left(T, x, v_{j}, j+1\right), \hat{g}^{2, j}\left(x, v_{j}, v_{j+1}\right)=x+g\left(T, \hat{g}^{1, j}\left(x, v_{j}\right), v_{j+1}, j+\right.$ $2), \ldots, \hat{g}^{k, j}\left(x, v_{1}, \ldots, v_{k}\right)=x+g\left(T, \hat{g}^{k-1, j}\left(x, v_{j}, \ldots, v_{j+k-1}\right), v_{j+k}, j+k\right)$, and define now $g^{k, j}$ by $g^{k, j}(x):=\inf _{v_{j}, \ldots, v_{j+k}} \hat{g}^{k, j}\left(x, v_{j}, \ldots, v_{j+k}\right)$, (actually essinf almost sure infimum - should be used).

\section{Remark 14 Restriction on the time path}

Consider the end constrained optimization problem [9]-[11], [39] [40], [42] with the added restriction that there are given open sets $\hat{Q}(j) \subset(0, T) \times \mathbb{R}^{n}$ in which admissible solutions $x\left(t ; \tau_{1}, \ldots \tau_{j}\right)$ are required to stay, $j=0,1, \ldots$, (i.e. $\left.\left(t, x\left(t ; \omega^{j}\right)\right) \in \hat{Q}(j)\right)$. (This amounts to an additional requirement on solutions to be called admissible.) Controls $u\left(t ; \tau_{1}, \ldots, \tau_{j}\right), j=0,1, \ldots$ are admissible if the corresponding solutions are admissible. Theorem 6 applies even to the present problem in the sense that it yields optimality in the present class of admissible controls provided $(t, x(t ; s, y, j)) \in \hat{Q}(j)$ for all $t \geq s$, all $(s, y) \in Q^{0}(j)$. (Of course, it is now understood that in the definition of the sets $Q(j)$ in [20], the solutions $x(., \omega)$ are admissible in the present sense).

\section{Remark 15 Soft terminal constraints}

Consider the problem were, for simplicity, $h_{0}=0$ and the hard end conditions [39] are replaced by the following soft ones:

$$
E\left[x_{i}(T-, \omega)\right]=\hat{x}_{i}, \quad i=1, \ldots, n^{\prime}
$$




$$
E\left[x_{i}(T-, \omega)\right] \geq \hat{x}_{i}, \quad i \geq n^{\prime}+1, \ldots, n^{\prime \prime}
$$

Consider the solution procedure for problem [9]-[11], [39], [40]. When [39] is replaced by [50], the condition [43] in this procedure has to be replaced by the following one. Define an auxiliary scrap value function $h_{0}=\sum_{i=1}^{n} \tilde{\lambda}_{i} x_{i}$, where the multipliers $\tilde{\lambda}_{i}$, so far are unknowns. Apply the solution procedure for this $h_{0}$-function, and at the end, determine the $\tilde{\lambda}_{i}$ 's by using the conditions [50], and [51] below: For some non-negative multipliers $\hat{\lambda}_{i}, i=1, \ldots, n$,

$$
\begin{gathered}
\tilde{\lambda}_{i} \geq 0, i=n^{\prime}+1, \ldots, n^{\prime \prime}, \\
\tilde{\lambda}_{i}=0 \text { if } E\left[x_{i}(T ; \omega)\right]>\hat{x}_{i}, i=n^{\prime}+1, \ldots, n^{\prime \prime} \\
\hat{\lambda}_{i}=0, i=n^{\prime \prime}+1, \ldots, n .
\end{gathered}
$$

In [50] and [51 b], $x(., \omega)$ is the nonanticipating solution starting at $\left(0, x^{0}\right)$, constructed by the method described in Remark 2. Note that in the present case, no controllability condition like that in [45] is needed.

Related to Theorems 6 and 3, there is a sufficient condition based on the so-called HJB-equations of the problem. To introduce this approach, let us make some introductory comments. We need the following convention: A function defined on a possibly non-open set $A$ is $C^{1}$ on $A$ if it has an extension to a larger open set which is $C^{1}$. Define the optimal value function by

$$
\begin{aligned}
& J^{*}(s, y, j)=\max _{u(., .)}\left\{E \left[\sum_{i} \int_{\min \left\{T, \tau_{i}\right\}}^{\min \left\{T, \tau_{i+1}\right\}} 1_{[s, T]}(t) f_{0}(t, x(t, \omega), u(t, \omega), i) d t+\right.\right. \\
& \left.\left.\sum_{s \leq \tau_{i}<T} g_{0}\left(\tau_{i}, x\left(\tau_{i}-, \omega\right), i\right)+h_{0}(x(T-, \omega)) \mid s, y, j\right]\right\} .
\end{aligned}
$$

where the maximization is over all $(s, y, j)$-admissible controls $u(., \omega)$, and where $x(t, \omega):=x^{u(. . .)}(t, \omega)$. As before, a $(s, y, j)$-admissible control is a nonanticipating control for which $x(t, \omega)=x^{u(., .)}(t, \omega)$ satisfies the terminal conditions and starts in $(s, y, j),(j$ jumps have already occurred). Without being too precise, let it be noted that very often, especially in the free end case, for each $j, J^{*}(s, y, j)$ is $C^{1}$ in (an open set containing) $\operatorname{cl} Q(j)$ and satisfies the " $j$-th" HJB-equation:

$$
\begin{gathered}
0=J_{s}(s, y, j)+\max _{u \in U}\left\{f_{0}(s, y, u, j)+J_{y}(s, y, j) f(s, y, u, j)\right\} \\
+\lambda(j+1)\left\{g_{0}(s, y, j+1)+J(s, y+g(s, y, j+1), j+1)-J(s, y, j)\right\} .
\end{gathered}
$$


In the free end case, the boundary condition on $J(s, y, j)$ is

$$
J(T, x, j)=h_{0}(x) .
$$

In the case where a maximum of $N$ jumps can occur, there is a recursive procedure available for solving the HJB-equation. First [53]-[54] are solved for $j=N$, then for $j=N-1$, (then we need the knowledge of $J(t, x, N)$ ), and next for $j=N-2$, (then we need $J(t, x, N-1)$ ), and so on backward. In end constrained cases, [55] below is used instead of [54], (then $J(s, y, j)$ is perhaps not meaningfully defined for $s=T$ ).

Related to the HJB-equation is a sufficient condition, or verification theorem. We need the following boundary conditions.

$$
h_{0}\left(x^{*}(T, \omega)\right)=\lim _{s \rightarrow T} J\left(s, x^{*}(s, \omega), j\right) \text { for all } \omega=\left(\tau_{1}, \tau_{2}, \ldots\right),
$$

for which $\tau_{j}<T \leq \tau_{j+1}$

For all admissible solutions $\hat{x}(t ; \omega), \lim _{s \rightarrow T} J(s, \hat{x}(t ; \omega), j) \geq h_{0}(\hat{x}(T, \omega))$,

for all $\omega=\left(\tau_{1}, \tau_{2}, \ldots\right)$ for which $\tau_{j}<T \leq \tau_{j+1}$.

For the next theorem, let us postulate that the system satisfies the properties stated subsequent to Theorem 1.

Theorem 7 Assume that $J(s, y, j), j=0,1, \ldots$, are $C^{1}$ functions on $Q(j)$ satisfying the HJB-equation [53] for all $(s, y) \in Q(j)$, and the boundary condition [55 b]. Assume that for any admissible solution $x(t, \omega),|J(t, x(t, \omega), j)| \leq$ $\alpha_{J}+\kappa_{J}|x(t, \omega)|$, and that $s \rightarrow J(s, x(s, \omega), j)$ for $s \in\left(\tau_{j}, \tau_{j+1}\right)$ is Lipschitz continuous with rank $\hat{\kappa}_{J}\left(1+\kappa_{g}\right)^{j}$, for some constant $\hat{\kappa}_{J}$. (The constants $\alpha_{J}, \kappa_{J}$, and $\hat{\kappa}_{J}$ may depend on $x(.,$.$) .) Finally, assume that there exists an$ admissible pair $\left(x^{*}(s, \omega), u^{*}(s, \omega)\right)$ satisfying [55 a] and the equality

$\max _{u \in U} H\left(t, x^{*}(t, \omega), u, J_{x}^{*}\left(t, x^{*}(t, \omega), j\right), j\right)=H\left(t, x^{*}(t, \omega), u^{*}(t, \omega), J_{x}^{*}\left(t, x^{*}(t, \omega), j\right), j\right)$

for all $\omega$ such that $\tau_{j}<t \leq \tau_{j+1}$. Then $u^{*}(t, \omega)$ is optimal.

The condition [55] is automatically satisfied if $J(s, y, j)$ can be continuously extended to $Q(j) \bigcup\left(\left(\{T\} \times \mathbb{R}^{n}\right) \cap \mathrm{cl} Q(j)\right), j=0,1, \ldots$, and [54] holds.

The relationship between Theorems 6 and 7 is as follows. A standard manner of solving the HJB-equation is using the so-called characteristic equations. Defining $\hat{H}(s, x, p)=\max _{u}\left\{f_{0}(s, x, u)+p f(s, x, u)\right\}$, the so-called 
characteristic equations for the solution of the $j$-th first order partial differential HJB-equation is $\dot{x}(s)=\hat{H}_{p}(s, x, p), \dot{p}(s)=-\hat{H}_{x}(s, x, p)-\lambda(j+$ $1)(\partial / \partial x)\left\{g_{0}(s, x, j+1)+J(s, x+g(s, x, j+1), j+1)-J(s, x, j)\right\}$. It can be shown that these two equations imply that $p(s ; s, y, j)=J_{y}(s, y, j)$, so the latter equation can alternatively be written $\dot{p}(s)=-\hat{H}_{x}(s, x, p)+\lambda(j+$ 1) $\left[p-g_{0 x}(s, x, j+1)-p(s ; s, x+g(s, x, j+1), j+1)\left(I+g_{x}(s, x, j+1)\right)\right]$. If $\hat{H}$ is $C^{1}$ in $x$, the equation for $x$ and the last equation for $p$ can be shown to be the same as [13]-[14] above. When all $x(t ; s, y, j)$ and $p(t ; s, y, j)$ have been found, from [47], [48] functions $J(s, y, j)$ are obtained, and they will satisfy the HJB-equation [53].

When using the HJB-equation(s), we always get candidate controls on Markov form, namely as any control function that yields the maximum in the HJBequation.

Sometimes, it is possible to use Theorem 7 (but not Theorem 6), in cases where open sets $Q^{0}(j)$ cannot be found, for which the conditions in Theorem 6 are satisfied. Of course, a method of producing the functions $J(s, y, j)$ of Theorem 7 in case $N<\infty$ is to use characteristic solutions, together with the functions $z(t ; s, y, j)$ and put $J(s, y, j)=z(s ; s, y, j)$, see [47], [48]. We can hope that these solutions can be defined for $(s, y) \in Q(j)$. Finally, we can check if $J(s, y, j)$ has a $C^{1}$-extension to some larger open set.

\section{Remark 16 Infinite number of jumps, all $\lambda(i)$ equal}

When $N=\infty$, there is an infinite family of HJB-equations [53]. So, assume not only that $N=\infty$, but also that all $\lambda(i)=\lambda$ and that $g_{0}, g, f_{0}, f$ are independent of $j$. Then evidently, $J^{*}(s, y, j)$ is independent of $j$, and the family of HJB-equations reduces to one, namely the one obtained from [53] by deleting the arguments $j$ and $j+1$.

Whether all the $\lambda$ 's are equal or not, it is only in rare cases that explicit formulas for solutions of the HJB-equation(s) can be found.

In the present case, the adjoint equations also reduce to a single equation.

In the case of this remark, Davis (1993) has discussed the usefulness of the HJB-equation for numerical solutions of the problem. He also considers HJBequations in problems with additional features, for example problems where the state jumps each time it reaches a boundary. 
Example 6 (The following problem is essentially Example 1, with $N=\infty$.) Consider the problem:

$$
\max E\left[\int_{0}^{T}-u^{2} / 2 d t+a x(T)\right], \quad \dot{x}=u \in \mathbb{R}, x(0)=0,
$$

with a possibility for an infinite number of upwards jump in $x(t)$ with the same intensity $\lambda$ for all jumps $\tau_{j}$, and with the same size $b$, (i.e. $x\left(\tau_{i}+\right)-$ $\left.x\left(\tau_{i}-\right)=b\right)$.

The single HJB-equation is $0=J_{s}+\max _{u}\left[-u^{2} / 2+J_{y} u\right]+\lambda[(J(s, y+b)-$ $J(s, y)]$. The maximum is calculated by putting the first derivative of $u$ equal to zero, it yields the maximum point $u=J_{y}$. Inserting this in the HJB-equation gives $0=J_{s}+\left(J_{y}\right)^{2} / 2+\lambda[(J(s, y+b)-J(s, y)]$. We guess that $J(s, y)$ is of the form $\phi(s)+k x, k$ a constant. The HJB-equation yields that $0=\phi^{\prime}+k^{2} / 2+\lambda k b$, so $\phi(s)=c s+C$, where $c:=-k^{2} / 2-\lambda k b$, and $C$ is an integration constant. Now, $a x=\phi(T)+k x=c T+C+k x$, so $k=a, C=-c T$, hence $\phi(s)=c(s-T)$. The control is $u=J_{y}=a$.

\section{Remark 17 (Infinite horizon, infinite number of possible jumps)}

In this remark, we shall briefly comment upon a sufficient condition for optimality in the case where $T=\infty, h_{0} \equiv 0, N=\infty$ (an infinite number of possible jumps), all $\lambda(j)=\lambda$, and where $g_{0}, g, f_{0}$, and $f$ are independent of $j$. Then, for all $j, J(s, x, j)=J(s, x, 0)$, and the conclusion of Theorem 7 still holds provided the boundary conditions [55] are replaced by the following conditions.

$$
0=\limsup _{s \rightarrow \infty} E J\left(s, x^{*}(s, \omega), 0\right)
$$

For all admissible solutions $\hat{x}(t ; \omega), \liminf _{s \rightarrow \infty} E J(s, \hat{x}(s ; \omega), 0) \geq 0$.

A special case is where, in addition, $g_{0}(t, x)=e^{-\beta t} g^{0}(x), f_{0}(t, x, u)=e^{-\beta t} f^{0}(x, u), \beta>$ 0 , and where $g$ and $f$ are independent of $t$. Then $J(s, x, 0)=e^{-\beta t} \tilde{J}(x)$, where $\tilde{J}(x)$ satisfies the "current value HJB-equation" $0=-\beta \tilde{J}+\max \left\{f^{0}+\tilde{J}_{y} f\right\}+\lambda\left\{g^{0}+\tilde{J}(y+g(y))-\tilde{J}(y)\right\}$. 


\section{Example 7}

$$
\max \int_{0}^{\infty}(x u)^{\alpha} e^{-\beta t} d t
$$

subject to

$$
d x / d t=a x(1-u), x(0)=x^{0}>0, u \in(0, \infty) \text { and } x(\tau+)=k x(\tau-),
$$

where an infinite number of jumps with intensity $\lambda$ can occur. It is assumed that $\alpha \in(0,1), \beta>0, a>0, k>0$, and $\kappa:=\beta-\lambda\left(k^{\alpha}-1\right)-\alpha a>0$.

We guess that $\tilde{J}=A y^{\alpha}, A>0$. Then the current value HJB-equation reduces to $0=-\beta y^{\alpha}+\max _{u>0}\left[y^{\alpha} u^{\alpha} / A+\alpha y^{\alpha} a(1-u)\right]+\lambda\left(k^{\alpha}-1\right) y^{\alpha}$. Here, $y^{\alpha}$ can be cancelled, and the maximization gives $u=B$, where $B=(a A)^{1 /(\alpha-1)}$, so the HJB-equation reduces to $0=-\beta+B^{\alpha} / A+\alpha a(1-B)+\lambda\left(k^{\alpha}-1\right)=$ $-\kappa+a(1-\alpha) B$, or $\kappa=(1-\alpha) \gamma A^{1 /(1-\alpha)}, \gamma:=a^{\alpha /(\alpha-1)}$. Hence, $A=$ $(\kappa / \gamma(1-\alpha))^{\alpha-1}$, and $\left.B=a^{1 /(\alpha-1)} \kappa / \gamma(1-\alpha)\right) \kappa / a(1-\alpha)$. All admissible $x(t, \omega)$ are nonnegative, so $\left[55^{*} b\right]$ is evidently satisfied. Moreover, $d x / d t=c x$, for $c=a(1-B)$. Note that for each $t$, such that $\tau_{j}<t \leq \tau_{j+1}$, for $t \in\left(\tau_{j}, \tau_{j+1}\right)$, $x\left(t-, \tau_{1}, \ldots, \tau_{j}\right)=x\left(\tau_{j}+\right) e^{c\left(t-\tau_{j}\right)}=k x\left(\tau_{j}-\right) e^{c\left(t-\tau_{j}\right)}$. Using this equality for $j$ replaced by $j-1, j-2, \ldots, 0$, we get $x\left(t-, \tau_{1}, \ldots, \tau_{j}\right)=k^{j} x^{0} e^{c t}$. From the theory of Poisson processes, we know that the probability of $j$ jumps in an interval $[0, t]$ is $(\lambda t)^{j} e^{-\lambda t} / j$ !. Hence, we get that the expected value $E J\left(t, k^{j} x^{0} e^{c t}\right)$, for $m=k^{\alpha}$, equals $A E\left[\left(m^{j}\left(x^{0}\right)^{\alpha} e^{\alpha c t-\beta t}\right]=A\left(x^{0}\right)^{\alpha} e^{(\alpha c-\beta) t} \sum_{0}^{\infty}(m \lambda t)^{j} e^{-\lambda t} / j !=\right.$ $A\left(x^{0}\right)^{\alpha} e^{(\alpha c-\beta) t} e^{m \lambda t} e^{-\lambda t}=A\left(x^{0}\right)^{\alpha} e^{(\alpha c+(m-1) \lambda-\beta) t}=A\left(x^{0}\right)^{\alpha} e^{-\kappa(1-\alpha)^{-1} t}$. The coefficient in front of $t$ is negative, hence, the limit of the last expression, as $t$ goes to infinity, is zero, so $\left[55^{*} a\right]$ holds. The optimal (Markov) control is $u(s, y)=B$, thus independent of $(s, y)$.

\section{Remark 18 (Generalizations (dependence on $\omega$ ))}

The functions $f_{0}, f, g_{0}$, and $g$ can be allowed to depend on $\omega$, provided they are nonanticipating in $(t, \omega)$ for each $x, u$. Then the explicit dependence on $\omega$ must be entered in all formulas. This is done by replacing all lower case $j$ 's in the formulas by $\omega^{j}$. In particular, $Q(j)$ becomes $Q\left(\omega^{j}\right)$. Then the conditions subsequent to Theorem 1 must hold uniformly in $\omega$, i.e. the inequality involving $\phi$ must hold for all $\omega$, and the uniform continuity of $x \rightarrow f_{x}\left(t, x, u^{*}(t, \omega), \omega\right)$ must be uniform in $(t, \omega)$.

Instead of assuming that [19] holds for $\phi=f_{0}$ and $\phi=f$, then, when $B(0, n)$ in [19] is replaced by $B(0, n)+\left\{u^{*}(t)\right\}$ and $|x|$ by $\left|x-x^{*}(t)\right|$, it can be assumed that this modified condition [19] holds for $\phi=f_{0}(t, x, u, \omega)-$ 
$f_{0}\left(t, x^{*}(t, \omega), u^{*}(t, \omega), \omega\right)$, and $\phi=f(t, x, u, \omega)-f\left(t, x^{*}(t, \omega), u^{*}(t, \omega), \omega\right)$. In this case, replace $B(0, c)$ in [45] by $B(0, c)+\left\{u^{*}(t, \omega)\right\}$.

In this case, $u^{*}(t, \omega)$ need not be bounded, if only

$E \int_{0}^{T}\left[\left|f_{0}\left(t, x^{*}(t, \omega), u^{*}(t, \omega), \omega\right)\right|+\left|f\left(t, x^{*}(t, \omega), u^{*}(t, \omega), \omega\right)\right|\right] d t<\infty$.

\section{Remark 19 (Weakened differentiability assumptions on the char- acteristic solutions.)}

The differentiability conditions on $x(t ; s, y, j), p(t ; s, y, j), \hat{u}(t, x, p, j)$ and $\hat{H}(t, x, p, j)$ in Theorems 3 and 6 can be weakened as follows. Let $\phi_{k}(t, x, p, j), k=$ $1, \ldots, k_{j}^{*}$, be $C^{1}$-functions in $(t, x, p)$ for each $j$ and assume that for any given $j$, for any $(s, y) \in Q^{0}(j)$, if

$$
\begin{aligned}
& t \in(s, T) \text { and } \phi_{k}(t, x(t ; s, y, j), p(t ; s, y, j), j)=0, \text { then } \\
& \phi_{k t}+\phi_{k x}(\partial / \partial t) x\left(t^{ \pm} ; s, y, j\right)+\phi_{k p}(\partial / \partial t) p\left(t^{ \pm} ; s, y, j\right) \neq 0
\end{aligned}
$$

the partial derivatives $\phi_{k t}, \phi_{k x}$ and $\phi_{k p}$ being evaluated at $(t, x(t ; s, y, j), p(t ; s, y, j), j)$. (The expression on the left-hand side in [56] is assumed to be either $>0$, for both the right and left limit, or $<0$ for both limits.) Assume that, for any $(t, x, p) \in Q^{*}(j), \phi_{k}(t, x, p)=0$ for at most one $k$. Assume also that for each $j,(t, x, p) \rightarrow \hat{u}(t, x, p, j)$ has a $C^{0} \times C^{1} \times C^{1}$-extension to an open set containing $\operatorname{cl} A$, for any set $A$ of the form $\cap_{i} \Phi^{i}, \Phi^{i}=\left\{(t, x, p) \in Q^{*}(j)\right.$ : $\left.\phi_{i}(t, x, p, j)>0\right\}$, or $\Phi^{i}=\left\{(t, x, p) \in Q^{*}(j): \phi_{i}(t, x, p, j)<0\right\}$, (the direction of the inequality sign may depend on $i)$. Assume that $\hat{H}(t, x, p, j)$ is $C^{2}$ in $\left\{(t, x, p) \in Q^{*}(j): \phi_{k}(t, x, p, j) \neq 0\right.$ for all $\left.k\right\}$. Let $Q^{1}(j):=\{(s, y) \in$ $Q^{0}(j): \phi_{k}(s, y, p(s ; s, y, j), j)=0$ for some $\left.k\right\}$, and $Q_{s, y, j}:=\{t \in[s, T):$ $\phi_{k}(t, x(t ; s, y, j), p(t ; s, y, j), j)=0$ for some $\left.k\right\}$, and assume that for any $(s, y) \in Q^{0}(j) \backslash Q^{1}(j)$, for any $t \notin Q_{s, y, j},\left(s^{\prime}, y^{\prime}\right) \rightarrow\left(x\left(t ; s^{\prime}, y^{\prime}, j\right), p\left(t ; s^{\prime}, y^{\prime}, j\right)\right)$ is $C^{1} \times C^{2}$ for all $\left(s^{\prime}, y^{\prime}\right)$ in a neighborhood $N_{t, s, y, j}$ of $(s, y)$. Define $J(s, y, j):=$ $z(s ; s, y, j)$ (see [47]) and assume that $M(s, y, j):=J(s, y+g(s, y, j+1), j+1)$ is $C^{0} \times C^{2}$ in $Q^{0}(j) \backslash Q^{1}(j)$, that $\sup _{t \in[s, T) \backslash Q_{s, y, j}}|M(t, x(t ; s, y, j), j)|$,

$\sup _{t \in[s, T) \backslash Q_{s, y, j}}\left|M_{x}(t, x(t ; s, y, j), j)\right|$ and $\sup _{t \in[s, T) \backslash Q_{s, y, j}}\left|M_{x x}(t, x(t ; s, y, j), j)\right|$ are locally bounded on $Q^{0}(j) \backslash Q^{1}(j)$, and that for any $(s, y) \in Q^{0}(j) \backslash Q^{1}(j)$, $\left(s^{\prime}, y^{\prime}\right) \rightarrow J\left(s^{\prime}, y^{\prime}+g\left(s^{\prime}, y^{\prime}, j+1\right), j+1\right)$ has a $C^{0} \times C^{2}$-extension to an open set around the set $\left\{\left(s^{\prime}, y^{\prime}\right)=\left(s^{\prime}, x\left(s^{\prime} ; s, y, j\right)\right): s^{\prime} \in[a, b]\right\}$, for any interval $[a, b] \subset(s, T)$ for which $(a, b) \cap Q_{s, y, j}=\emptyset$.

Finally, assume that for any admissible solution $x(., \omega)$, if $\left(s, x\left(s, \omega^{j}\right)\right) \in$ $\Phi_{k, j}:=\left\{(s, y) \in Q(j): \phi_{k}(s, y, p(s ; s, y, j), j)=0\right\}, s>\tau_{j}$, then for some $\epsilon>0,\left(s^{\prime}, x\left(s^{\prime}, \omega^{j}\right)\right) \notin \Phi_{k, j}$ for $s^{\prime} \in(s, s+\epsilon)$.

Note that in this Remark, $z(s ; s, y, j+1)$ is perhaps only $C^{0} \times C^{2}$ in 
$Q^{0}(j+1) \backslash Q^{1}(j+1)$, so, (more or less), the following recursive property must hold: $(s, y) \in Q^{0}(j) \backslash Q^{1}(j) \Rightarrow(s, y+g(s, y, j+1)) \in Q^{0}(j+1) \backslash Q^{1}(j+1)$. Thus, given the functions $\phi_{k}(., ., ., j+1)$, functions $\phi_{k}(., ., ., j)$ must be found such that also this property holds.

In the case of Remark 18, for the conclusions in Remark 19 to hold, it can be assumed that the above conditions hold for $j$ replaced by $\omega^{j}$, (the replacement is not carried out for $j$ in $k_{j}^{*}$ ), each $\phi_{k}$, separately, piecewise and right-continuous in each $\tau_{i}, i \leq j$. See Remark 27 below.

Remark $20\left(\lambda(j)=\lambda(t, x, u, j), g_{0}=g_{0}\left(t, x, V_{j}, w_{j}, j\right), g=g\left(t, x, V_{j}, w_{j}, j\right)\right)$

In this remark the intensities $\lambda(j)$ are allowed to depend on $(t, x, u)$, so $\lambda(j)=$ $\lambda(t, x, u, j)$, with $\lambda(., ., . .) \leq$.$K , for some K$. Moreover, $g_{0}$ and $g$ are assumed to depend on two additional variables $V_{j}, w_{j}$, so $g_{0}=g_{0}\left(t, x, V_{j}, w_{j}, j\right)$, $g=g\left(t, x, V_{j}, w_{j}, j\right)$. Here, $V_{j}$ is a stochastic variable in $R^{\hat{n}}$, with cumulative distribution $\pi(t, x, j)$. If jump number $j$ occurs at $t=\tau_{j}$ and the state before the jump is $x=x\left(\tau_{j}-, \omega\right)$, then the cumulative distribution of $V_{j}$ is $\pi(t, x, j)=\pi\left(\tau_{j}, x\left(\tau_{j}-, \omega\right), j\right)$. Furthermore, $w_{j}$ is a control variable taking values in a given set $W_{j}$. It is assumed that at each jump point $\tau_{j}$ it is possible to choose $w_{j}$ after having observed the "size" $v_{j}$ of the jump, and $w_{j}$ can also depend on all earlier $\tau_{i}, v_{i}, \tau_{i}<t$. Now, $\omega=\left(\tau_{1}, v_{1}, \tau_{2}, v_{2}, \ldots\right)$, and the controls $u(t, \omega)$ and $w_{j}(t, v, \omega)$ depend only on $\tau_{i}, V_{i}$ for $\tau_{i}<t$. The functions $g_{0}, g, g_{0 x}, g_{x}$ are continuous in $(x, V, w)$, uniformly in $t$, and piecewise and left-continuous in $t$.

Optimal non-anticipating controls are denoted $u^{*}(t, \omega), w_{j}^{*}(t, v, \omega)$. The HJB-equations are now

$$
\begin{gathered}
0=J_{s}(s, y, j)+\sup _{u \in U}\left\{f_{0}(s, y, u, j)+J_{y}(s, y, j) f(s, y, u, j)+\right. \\
\lambda(x, u, j+1) E\left[\operatorname { s u p } _ { w \in W _ { j + 1 } } \left\{g_{0}(s, y, V, w, j+1)+\right.\right. \\
(s, y+g(s, y, V, w, j+1), j+1)-J(s, y, j)\} \mid s, y, j]\} .
\end{gathered}
$$

The expectation is calculated by means of $\pi(s, y, j)$.

Moreover, provided $\lambda$ depends only on $(t, x, j)$, and $\pi$ only on $(t, j)$, the equations in the ("characteristic") solution method must be modified as follows: First, in addition to $\hat{u}$, we also need the function $\hat{w}(t, x, v, j+1)$ yielding, by 
assumption, the maximum in

$$
\max _{w \in W_{j}}\left[g_{0}(t, x, v, w, j+1)+z(t ; t, x+g(t, x, v, w, j+1), j+1) .\right.
$$

There are now three "states" $x, p, z$, whose differential equations must be solved simultaneously:

$$
\begin{aligned}
& \dot{x}=f(t, x, \hat{u}(t, x, p, j), j) \\
& \dot{p}=-p H_{x}(t, x, \hat{u}(t, x, p, j), j)+\lambda(t, x, j+1) p- \\
& \lambda(t, j+1)\left\{E \left[\left(t ; t, x+g\left(t, x, V_{j+1}, \hat{w}\left(t, x, V_{j+1}, j+1\right), j+1\right)(I+\right.\right.\right. \\
& \left.g_{x}\left(t, x, V_{j+1}, \hat{w}\left(t, x, V_{j+1}, j+1\right), j+1\right)\right)+g_{0}\left(t, x, V_{j+1}, \hat{w}\left(t, x, V_{j+1}, j+1\right), j+\right. \\
& 1) \mid t, j]\}+ \\
& \left\{z-E\left[g_{0}\left(t, x, V_{j+1}, \hat{w}\left(t, x, V_{j+1}, j+1\right), j+1\right)+\right.\right. \\
& \left.z\left(t ; t, x+g\left(t, x, V_{j+1}, \hat{w}\left(t, x, V_{j+1}, j+1\right), j+1\right) \mid t, j\right]\right\} \lambda_{x}(t, x, j+1), \\
& \dot{z}=\lambda(t, x, j+1)\left\{z-E\left[g_{0}\left(t, x, V_{j+1}, \hat{w}\left(t, x, V_{j+1}, j+1\right), j+1\right)+\right.\right. \\
& \left.z\left(t ; t, x+g\left(t, x, V_{j+1}, \hat{w}\left(t, x, V_{j+1}, j+1\right), j+1\right) \mid t, j\right]\right\} .
\end{aligned}
$$

The expectation is calculated by means of $\pi(t, j)$. For each $j$, these equations are solved simultaneously in $(x, p, z)$, with side conditions in the free end case $x(s)=y, p(T)=h_{0 x}(x(T)), z(T)=h_{0}(x(T))$ and in the hard end constrained case, [44] and $z(T)=h_{0}(x(T)$ ), (in which case $g$ is assumed to be independent of $w$ ).

For necessary conditions in the case $\lambda=\lambda(t, x, \omega)$, see Seierstad 2001.

\section{Optimal stopping problems}

In this section, control problems are studied in which (also) the terminal time is subject to choice. First, we describe the necessary changes in the basic solution method, and state a corresponding sufficient condition, based on the field of characteristics obtained. Next, the approach connected with the HJB-equation will be discussed.

The problem studied is now the maximization problem:

$\max _{u(.,), T \in\left[T_{1}, T_{2}\right]} E\left[\sum \int_{\min \left\{\tau_{j}, T\right\}}^{\min \left\{\tau_{j+1}, T\right\}} f_{0}(t, x, u, j) d t+\sum_{\tau_{j}<T} g_{0}\left(\tau_{j}, x\left(\tau_{j}-\right), j\right)+h_{0}(T, x(T-))\right]$

where the maximization is subject to the standard restrictions [9], [10], (the differential equation, the given start point, the jump condition, and the restriction $u \in U)$. A fixed interval $\left[T_{1}, T_{2}\right]$ is specified, within which $T$ is 
subject to choice. We mainly consider the case of a free end (no terminal conditions). Again jump intensities $\lambda(i)$ are specified, and the basic solutions procedure will only work if, for some $N<\infty, \lambda(i)=0$ for $i>N$.

The basic solution procedure will now be changed to allow for the optimal choice of $T$. It is assumed that $f_{0}, f, g_{0}, g$, and $h_{0}$ are $C^{2}$. Since $T$ is subject to choice, we expect $T$ to become a function of $(s, y, j), T=T(s, y, j) \in\left[T_{1}, T_{2}\right]$. For each starting point $(s, y, j)$, we shall also choose $T$. Since we have one more unknown, we need to add further conditions to the basic conditions [12]-[15] of the solution method. In addition, [14] needs modification. Let us write down all equations needed for the solution method. (In the method, we need an auxiliary state variable $z$ that we have met before.)

Solution method for free terminal time, free end, $N<\infty$ The solution method makes use of the following relationships.

$\hat{u}(t, x, p, j)$ maximizes $H(t, x, u, p, j):=f_{0}(t, x, u, j)+p f(t, x, u, j)$ for $u \in U$.

$$
\dot{x}(t)=f(t, x, \hat{u}(t, x, p, j), j),
$$

$$
\begin{gathered}
\dot{p}(t)=-H_{x}(t, x, \hat{u}(t, x, p), p, j)+\lambda(j+1) p \\
-\lambda(j+1)\left[g_{0 x}(t, x, j+1)+J_{x}(t, x+g(t, x, j+1), j+1)\left(I+g_{x}(t, x, j+1)\right)\right][60]
\end{gathered}
$$

with boundary conditions

$$
x(s)=y, p(T)=\left[h_{0 x}(T, x)\right]_{x=x(T ; s, y, T, j)}
$$

$$
\begin{aligned}
& \dot{z}(t)=\lambda(j+1)\left[z-g_{0}(t, x, j+1)-J(t, x+g(t, x, j+1), j+1)\right]- \\
& f_{0}(t, x, \hat{u}(t, x, p, j), j), z(T)=h_{0}(T, x(T ; s, y, T, j))
\end{aligned}
$$

$$
\begin{gathered}
\eta(T, x(T, s, y, T, j), p(T, s, y, T, j), j)=0 \text { if } T \in\left(\max \left\{s, T_{1}\right\}, T_{2}\right), \\
\eta(T, x(T, s, y, T, j), p(T, s, y, T, j), j) \geq 0 \text { if } T=T_{2}, \\
\eta(T, x(T, s, y, T, j), p(T, s, y, T, j), j) \leq 0 \text { if } T=\max \left\{s, T_{1}\right\}, \\
\text { where } \eta(T, x, p, j):=\max _{u} H(T, x, u, p, j)+h_{0 t}(T, x)+ \\
\lambda(j+1)\left[g_{0}(T, x, j+1)+J(T, x+g(T, x, j+1), j+1)-h_{0}(T, x)\right]
\end{gathered}
$$




$$
\begin{gathered}
J(s, y, T, j):=z(s ; s, y, T, j), \text { and } \\
J(s, y, j)=J(s, y, T(s, y, j), j), \text { for } s<T(s, y, j), J(s, y, j) \\
=h_{0}(s, y) \text { for } s=T(s, y, j) .
\end{gathered}
$$

Condition [64] contains only definitions: we need the entity $J(s, y, j+1)$ in [60] and [62]. Next, $T$ is a parameter that is determined in the solution procedure. Before knowing the correct value of $T$, we need to solve [59]-[61], for arbitrary $T$, (as well as arbitrary $(s, y, j)$ ), so we write the solution pair as $(x(t ; s, y, T, j), p(t ; s, y, T, j))$. Moreover, there is not a full simultaneity between the differential equations. In fact when $x(t ; s, y, T, j)$ and $p(t ; s, y, T, j)$ are known, we can insert these functions for $x$ and $p$ in [62], and solve this linear equation in $z$, to find the solution $z=z(t ; s, y, T, j)$. Once $x(t ; s, y, T, j)$ and $p(t ; s, y, T, j)$ are known, we can use [63] to determine $T=T(s, y, j)$. A full explanation of the use of these equations, are thus as follows, (again a recursive solution procedure is available). First, ("step $\left.N^{\prime \prime}\right), x(t ; s, y, T, N), p(t ; s, y, T, N)$ are found using [59]-[61], where $T$ is a given parameter. At this step $\lambda(N+1)=0$. Condition [62] is used to determine $z(t ; s, y, T, N)$ and condition [63] is used to determine the (hopefully) optimal $T=T(s, y, N)$, Then, $J(s, y, T, N)$ and $J(s, y, N)$ are written down, using [64]. Next, ("step $N-1$ "), from [59]-[61], $x(t ; s, y, T, N-1$ ), $p(t ; s, y, T, N-1)$ are found, (in [60] the function $J(s, y, N)$ just constructed enters). Again, using the known $J(s, y, N)$, [62] yields $z(t ; s, y, T, N-1)$, Moreover, using [63] (and the known $J(s, y, N)$ ), the optimal $T=T(s, y, N-$ $1)$ is determined. Then, $J(s, y, T, N-1)$ and $J(s, y, N-1)$ are written down, using [64]. Next, ("step $N-2$ "), from [59]-[61], $x(t ; s, y, T, N-$ 2), $p(t ; s, y, T, N-2)$ are found (in [60] the known function $J(s, y, N-1)$ enters). Then $z(t ; s, y, T, N-2)$ is found by means of [62], using the known $J(s, y, N-1)$. Moreover, inserting the known $J(s, y, N-1)$ and using [63], the optimal $T=T(s, y, N-2)$ is determined. Then, $J(s, y, T, N-2)$ and $J(s, y, N-2)$ are written down, using [64]. And so on. At each step quintuples $x(t ; s, y, T, j), p(t ; s, y, T, j), z(t ; s, y, T, j), T(s, y, j), J(s, y, j), j=$ $N, N-1, \ldots$, are obtained.

We call the collection $x(t ; s, y, j):=x(t ; s, y, T(s, y, j), j), p(t ; s, y, j):=$ $p(t ; s, y, T(s, y, j), j), J(s, y, j), T(s, y, j)$ a characteristic quadruple, and if we add $u(t ; s, y, j):=\hat{u}(t ; x(t ; s, y, j), p(t ; s, y, j))$, we speak of a characteristic quintuple. Recall that $s \in\left[0, T_{2}\right]$, and that $s \leq T(s, y, j) \leq T_{2}$. The function $u(t ; s, y, j)$ is defined for $t \in[s, T(s, y, j)]$, and the same goes for $x(t ; s, y, j)$, $p(t ; s, y, j)$. 
Also in the present situation, using the characteristic solutions $x(t ; s, y, j)$, we can write down the corresponding non-anticipating solution $x(t, \omega)$, and then the corresponding candidate control $u(t, \omega)$ (and also $p(t, \omega)$ ). The candidate stopping time $T(\omega)$ is defined as follows: Define first $T_{j}\left(\tau_{j}\right):=$ $T\left(\tau_{j}, x\left(\tau_{j}-, \omega\right)+g\left(\tau_{j}, x\left(\tau_{j}-, \omega\right), j\right)\right), j=1,2, \ldots$. Next, define $T_{0}\left(\tau_{0}\right)=T\left(0, x^{0}, 0\right)$, $T(\omega)=\min \left\{T_{j}\left(\tau_{j}\right): T_{j}\left(\tau_{j}\right)<\tau_{j+1}, j=0,1, \ldots\right\}$. Note that $T=T(\omega)$ only depends on $\tau_{i}$ 's that are $\leq T$, in other words, the function $1_{\left(T(\omega), T_{2}\right]}(t)$ is non-anticipating.

Remark 21 The formulation of [63] is chosen such that it also works in case of end constraints, briefly treated in Remark 23 below. In the free end case $\eta^{* *}(T, x, s, y, j):=\eta(T, x, p(T ; s, y, T, j), j)=$ $f_{0}(T, x, \hat{u}(T, x, p(T ; s, y, T, j), j), j)+$ $h_{0 x}(T, x) f(T, x, \hat{u}(T, x, p(T ; s, y, T, j), j), j)+h_{0 t}(T, x)+\lambda(j+1)\left[g_{0}(T, x, j+\right.$ $\left.1)+J(T, x+g(T, x, j+1), j+1)-h_{0}(T, x)\right]$.

Defining $\eta^{*}(T, s, y, j)=\eta^{* *}(T, x(T ; s, y, T, j), s, y, j)$, it is easily seen (and shown below) that $\eta^{*}(T, s, y, j)=J_{T}(s, y, T, j)$. Thus, assuming that $J(s, y, T, j)$ gives the optimal value when stopping at $T$, then [63] is a necessary condition for choosing $T$ optimally, (e.g. if $T(s, y, j) \in\left(\max \left\{s, T_{1}\right\}, T_{2}\right)$, by necessity, $J_{T}=0$ at this point).

Below, two examples are presented showing the use of this procedure. Since we in particular want to describe the use of [63] in determining the optimal stopping time, we have chosen examples that are quite trivial, in particular no control $u$ appears.

Example 6 Consider a pure stopping problem (no control $u$ ):

$$
\max \operatorname{Ex}(T) \text {, when } T \in[0, b], \quad \dot{x}=e^{-t}, x(0)=0
$$

and where $x(t)$ can have two downwards unit jumps with intensity $\lambda, \lambda<1$, $-\ln (\lambda)<b, b$ a fixed number .

The procedure [58]-[64] will be used. Evidently, $x(t ; s, y, T, 2)=y+e^{-s}-e^{-t}$, $p(t ; s, y, T, 2)=1$. Furthermore, $\eta(T, x, p, 2)$ equals $e^{-T} p$ so $\eta^{*}(T, s, y, 2):=$ $\eta\left(T, x(T, s, y, 2, p(T, s, y, 2), 2)=e^{-T}\right.$. Moreover, $z(t ; s, y, T, 2)=z(T ; s, y, T, 2)=$ $y+e^{-s}-e^{-T}, J(s, y, T, 2)=y+e^{-s}-e^{-T}$. In this case we always choose to wait until $t=b$ before stopping, a trivial result $([63] \Rightarrow T=b)$. Note that $J(s, y, 2)=y+e^{-s}-e^{-b}$.

Next, let us consider $j=1$. In this case, $\dot{p}=\lambda p-\lambda$. With the end condition $p(T, s, y, T, 1)=1$, this gives $p(t ; s, y, T, 1)=1$. Furthermore, $\eta(T, x, p, 1):=e^{-T} p+\lambda\left(x-1+e^{-T}-e^{-b}-x\right)$. Hence, for $\eta^{*}(T, s, y, 1):=$ 
$\eta(t, x(T, s, y, T, 1), p(T, s, y, T, 1), 1)=e^{-T}+\lambda\left(-1+e^{-T}-e^{-b}\right)$, we have that $\eta^{*}(T, s, y, 1)=0$ if $e^{-T}(1+\lambda)=\lambda\left(1+e^{-b}\right)$, i.e. $T=T^{*}=T^{*}(b):=$ $-\ln \left[\lambda\left(1+e^{-b}\right)(1+\lambda)^{-1}\right] \in(0, b)$. (If $e^{-b}=\lambda, T^{*}(b)=b$, as $d T^{*}(b) / d b \in$ $(0,1), T^{*}(b)<b$ for greater $b$ 's, those satisfying $e^{-b}<\lambda$.) Now, if $T<$ $T^{*}, \eta^{*}(T, s, y, 1)>0$, and if $T>T^{*}, \eta(T, s, y, 1)<0$. Hence, we cannot have $T(s, y, 1)=0$ or $T(s, y, 1)=b$, the only possibility is $T(s, y, 1)=$ $T^{*}$. Now, $\dot{x}=e^{-t}$ again gives $x(t ; s, y, T, 1)=y+e^{-s}-e^{-t}$, and $\dot{z}(t)=$ $\lambda\left[z-\left(x-1+e^{-t}-e^{-b}\right)\right]=\lambda\left[z-\left(x(t ; s, y, T, 1)-1+e^{-t}-e^{-b}\right)\right]=\lambda[z-$ $\left.\left(y-1+e^{-s}-e^{-b}\right)\right]$, so $z(t ; s, y, T, 1)=y+e^{-s}-1-e^{-b}+C e^{\lambda t}$ where $C$ is determined by $z(T ; s, y, T, 1)=x(T ; s, y, T, 1)$. The last equality gives $y+e^{-s}-1-e^{-b}+C e^{\lambda T}=y+e^{-s}-e^{-T}$, which gives $C=e^{-\lambda T}\left(1+e^{-b}-\right.$ $\left.e^{-T}\right)$, so $z(t ; s, y, T, 1)=y-1+e^{-s}-e^{-b}+\left(1+e^{-b}-e^{-T}\right) e^{\lambda(t-T)}$. Hence, $J(s, y, T, 1)=y-1+e^{-s}-e^{-b}+\left(1+e^{-b}-e^{-T}\right) e^{\lambda(s-T)}$ and for $s<T^{*}$, $J(s, y, T(s, y, 1), 1)=y-1+e^{-s}-e^{-b}+\left(1+e^{-b}-e^{-T^{*}}\right) e^{\lambda\left(s-T^{*}\right)}$ while for $s \geq T^{*}, J(s, y, 1)=y$.

Finally, as before, $p(T, 0,0,0)=1$, (we now need only consider $(s, y)=$ $(0,0)$. Moreover, for $T \leq T^{*}, \eta(T, x, p, 0)=e^{-T}+\lambda\left\{x-2+e^{-T}-e^{-b}+(1+\right.$ $\left.\left.e^{-b}-e^{-T^{*}}\right) e^{\lambda\left(T-T^{*}\right)}-x\right\}=e^{-T}+\lambda\left\{-2+e^{-T}-e^{-b}+\left(1+e^{-b}-e^{-T^{*}}\right) e^{\lambda\left(T-T^{*}\right)}\right\}$. Hence, $\eta^{*}(T, 0,0,0):=\eta(T, x(T, 0,0,0), p(T ; 0,0,0), 0)=e^{-T}+\lambda\left\{-2+e^{-T}-\right.$ $\left.e^{-b}+\left(1+e^{-b}-e^{-T^{*}}\right) e^{\lambda\left(T-T^{*}\right)}\right\}$. For $T=T^{*}, \eta^{*}(T, 0,0,0)=\lambda\left(1+e^{-b}\right)(1+$ $\left.\left.\lambda)^{-1}-\lambda=\lambda\left(1+e^{-b}\right)(1+\lambda)^{-1}-1\right)<\lambda(1+\lambda)(1+\lambda)^{-1}-1\right)=0$. Moreover, for $\left.T<T^{*},(d / d T) \eta^{*}=-e^{-T}(1+\lambda)+\lambda^{2}\left(1+e^{-b}-e^{-T^{*}}\right) e^{\lambda\left(T-T^{*}\right)}\right) \leq-e^{-T^{*}}(1+$ $\lambda)+\lambda^{2}\left(1+e^{-b}-e^{-T^{*}}\right)=-\lambda\left(1+e^{-b}\right)(1+\lambda)^{-1}+\lambda^{2}\left(1+e^{-b}-\lambda\left(1+e^{-b}\right)(1+\right.$ $\left.\lambda)^{-1}\right)=0$. Thus, if $T^{* *}$ is the solution of $\eta^{*}(T, 0,0,0)=0$, then $T^{* *}<T^{*}$ and the optimal stopping time is $\max \left\{0, T^{* *}\right\}$. (Before this time, if it is greater than $0, \eta^{*}(T, 0,0,0)>0$, after this time, $\eta^{*}(T, 0,0,0)<0$. $)$

Both the sufficient conditions below, (Remark 22, and Theorem 8, with Remark 23), can be used to show optimality of this solution.

\section{Sufficient conditions and necessary conditions for free terminal time}

Define the set

$$
G_{j}:=\left\{(s, y) \in\left[T_{1}, T_{2}\right) \times \mathbb{R}^{n}: s<T(s, y, j)\right\} .
$$

This set may be called a continuation region: If the state at time $t$ belongs to this set, it is still some time left before we stop. Normally, $J(s, y, j)$ is the maximal expected reward obtained when starting at $(s, y, j)$, so, normally, we must have that

$$
G(j):=\left\{(s, y) \in\left[T_{1}, T_{2}\right) \times \mathbb{R}^{n}: J(s, y, j)>h_{0}(s, y)\right\}=G_{j} .
$$


(At a point $(s, y, j)$ where the strict inequality holds, we don't want to stop, i.e. $s$ is $<T(s, y, j))$.

We now turn to a sufficiency theorem, in which the following assumption is needed: For $t>T_{1}$, if the state crosses the boundary $\partial G_{j}$ of the set $G_{j}$, it does so non-tangentially, or "at a certain angel".

This non-tangentiality property is secured by the condition that for each $(s, y) \in \partial G_{j} \cap\left(T_{1}, T_{2}\right) \times R^{n}$, for all $u \in U$,

$$
J_{t}^{* *}(s, y, j)-h_{t}(s, y)+\left(J_{x}^{* *}(s, y, j)-h_{0 x}(s, y)\right) f(s, y, u, j) \neq 0 .
$$

Here, for the moment, we assume that $J$ is extendable as a $C^{1}$-function from $G_{j}$ to a larger open set containing $\operatorname{cl} G(j)$, this extension being denoted $J^{* *}$. Often, $J^{* *}$ is different from the true $J$-function outside $G_{j}$.

Also the following condition is needed.

$$
\begin{array}{r}
0 \geq h_{0 t}(s, y)+\sup _{u \in U}\left\{f_{0}(s, y, u, j)+h_{0 x}(s, y) f(s, y, u, j)\right\} \\
+\lambda(j+1)\left\{g_{0}(s, y, j+1)+J(s, y+g(s, y, j+1), j+1)-h_{0}(s, y)\right\}
\end{array}
$$

Let $Q(j)$ be defined as before, with the modification that the admissible $x(.,$.$) 's used in the construction has individual sets of definitions [0, T(\omega)]$.

Theorem 8 (Sufficient condition for characteristic solutions, $N<$ $\infty)$ Assume that $f_{0}, f, g_{0}, h_{0}$ and $g$ are $C^{2}$-functions and that $\hat{H}(t, x, p, j)$ and $\hat{u}(t, x, p, j)$ are, respectively, $C^{0} \times C^{2} \times C^{2}$ and $C^{0} \times C^{1} \times C^{1}$ in an open set $Q^{*}(j)$. Assume that a collection of open sets $D^{0}(j) \subset(0, T) \times$ $\mathbb{R}^{n}$ and a collection of solutions $x(t ; s, y, j), p(t ; s, y, j)$, with corresponding stopping times $T(s, y, j) \in\left[\max \left\{s, T_{1}\right\}, T_{2}\right]$ and controls $u(t, s, y, j)=$ $\hat{u}(t ; x(t ; s, y, j), p(t, s, y, j))$ have been found for $(s, y) \in D^{0}(j)$, for $j=0$ even for $(s, y)=\left(0, x^{0}\right)$, with $p(T(s, y, j) ; s, y, j)=h_{0 x}(T(s, y, j), x(T(s, y, j) ; s, y, j))$ and $x(t ; s, y, j)$ starting at $(s, y, j)$. Assume that the following conditions are satisfied: Define $z(t ; s, y, j)$ by

$$
\begin{array}{r}
\dot{z}(t)=\lambda(j+1)\left[z-g_{0}(t, x, j+1)-\right. \\
z(t ; t, x+g(t, x, j+1), j+1)]-f_{0}(t, x, u(t ; s, y, j), j),
\end{array}
$$

where $x=x(t ; s, y, j)$, with terminal condition $z(T(s, y, j) ; s, y, j)=$ $h_{0}(T(s, y, j), x(T(s, y, j) ; s, y, j))$. Define also $J(s, y, j)=z(s ; s, y, j)$ for $(s, y) \in$ 
$D^{0}(j)$, and $J(s, y, j)=h_{0}(s, y)$, for $(s, y) \in\left(\left[T_{1}, T_{2}\right] \times \mathbb{R}^{n}\right) \backslash D^{0}(j)$. The sets $D^{0}(j)$ satisfy the conditions that, for any $(s, y) \in D^{0}(j), T(s, y, j)>s$, and that $T(s, y, j)=\sup \left\{t:\left(t^{\prime}, x\left(t^{\prime} ; s, y, j\right)\right) \in D^{0}(j)\right.$ for $\left.t^{\prime} \in[s, t]\right\}>s$. Moreover, assume that for any admissible solution $x(., \omega),\left(s, x\left(s, \omega^{j}\right)\right) \in D^{0}(j)$ when $s<T_{1}$, Assume that [67] is satisfied for a suitable $C^{1}$-extension $J^{* *}$ of $J$, and that [68] holds for all $(s, y) \in\left(\left[T_{1}, T_{2}\right] \times \mathbb{R}^{n}\right) \backslash D^{0}(j)$. Assume, for all $(s, y) \in D^{0}(j), t \in[s, T(s, y, j)]$, that $(t, x(t ; s, y, j), p(t ; s, y, j))$ belongs to $Q^{*}(j)$, and for $s^{\prime} \in(s, t)$,

$\left(x\left(t, s^{\prime}, x\left(s^{\prime} ; s, y, j\right), j\right), p\left(t, s^{\prime}, x\left(s^{\prime} ; s, y, j\right), j\right)\right)=(x(t ; s, y, j), p(t ; s, y, j))$ and $T\left(s^{\prime}, x\left(s^{\prime} ; s, y, j\right), j\right)=T(s, y, j)$ if $T(s, y, j)>s^{\prime}$. Assume that either,

$$
T(s, y, j) \in\left(T_{1}, T_{2}\right) \text { or } T(s, y, j)=\max \left\{s, T_{1}\right\} \text { or } T(s, y, j)=T_{2},
$$

for all $(s, y) \in D^{0}(j)$, (in the sense that either the first property holds for all such $(s, y)$, or the second property holds for all such $(s, y)$, or the third one hold for all such $(s, y))$. Assume that $J(s, y, j)>h_{0}(s, y)$ for $(s, y) \in D^{0}(j) \cap$ $Q(j), s \in\left[T_{1}, T_{2}\right)$ and that $\eta(T(s, y, j), x(T(s, y, j) ; s, y, j), p(T(s, y, j) ; s, y, j), j)=$ 0 if $T(s, y, j) \in\left(\max \left\{s, T_{1}\right\}, T_{2}\right)$, (for $\eta$, see [63]). Assume that in $D^{0}(j)$, $(s, y) \rightarrow(x(t ; s, y, j), p(t ; s, y, j), T(s, y, j))$ is $C^{1} \times C^{2}$. Moreover, assume that

$$
\hat{u}(t, x, p, j) \text { is bounded on bounded subsets of } Q^{*}(j) \text {. }
$$

Finally, assume that $p(s ; s, y+g(s, y, j+1), j+1)$ and $p_{x}(s ; s, y+g(s, y, j+$ $1), j+1)$ are bounded on bounded subsets of $D^{0}(j)$. Then the family of characteristic quadruples (with corresponding controls $u(t ; s, y, j)=$ $\hat{u}(t, x(t ; s, y, j), p(t ; s, y, j))$ is optimal. (The family yields an optimal nonanticipating control $u(t, \omega)$, and an optimal stopping time $T(\omega)$.)

Remark 21* If $\eta^{*}(T, s, y, j):=\eta(T, x(T ; s, y, T, j), p(T ; s, y, T, j), j)>0$ for $T \in(s, T(s, y, j))$, then $J(s, y, j)>h_{0}(s, y)$ in $D^{0}(j)$.

It is possible to weaken the differentiability assumptions in Theorem 8 to those of Remark 19, and weaken condition [68*]. For details see Theorem 16 below. (Typically, when $T(s, y, j)$ moves, say, towards $T_{2}$, one looses differentiability of $T(s, y, j)$, such situations must be "passed over rapidly", that is assumed to be the case in Theorem 16.)

Remark 22. A sufficient condition based on concavity can also be stated. We state only the most essential conditions: Assume that the concavity conditions in Remark 6 are satisfied. Moreover, for each $j$, assume that for the $T(s, y, j)$ obtained in the solution procedure, we have that 
$\eta(T, x(T, s, y, T, j), p(T, s, y, T ; j)) \geq 0$ if $T \in\left[\max \left\{T_{1}, s\right\}, T(s, y, j)\right)$, $\eta(T, x(T, s, y, T, j), p(T, s, y, T, j)) \leq 0$ if $T \in\left(T(s, y, j), T_{2}\right]$. Then the characteristic solutions are optimal.

Example 8 Again, for simplicity, we shall consider a pure stopping problem (no control $u$ ):

$$
\max \operatorname{Ex}(T), \text { when } T \in[0, b], \quad \dot{x}=-x, \quad x(0)=x^{0}>0
$$

and where $x(t)$ can have a single unit upwards jumps with intensity $\lambda>x^{0}$. The state $x$ is always positive, so all start values $y$ are chosen to be $>0$. After one jump, it surely pays to stop at once, $J(s, y, 1)=y$, and $p(t ; s, y, 1)=1$. Let us find $x\left(t ; 0, x^{0}, T, 0\right), p\left(t ; 0, x^{0}, T, 0\right)$. Evidently, $x\left(t ; 0, x^{0}, T, 0\right)=x^{0} e^{-t}$ and $\dot{p}=p+\lambda p-\lambda$. With $p(T)=1$, this gives $p\left(t, 0, x^{0}, T, 0\right)=[1-\lambda /(1+$ $\lambda)] e^{(1+\lambda)(t-T)}+\lambda /(1+\lambda)$. Next, $\eta(T, x, p, 0)$ in $[49]$ reduces to $\eta(T, x, p, 0):=$ $-x p+\lambda(x+1-x)=-x p+\lambda$, so $\eta^{*}\left(T, 0, x^{0}, 0\right):=\eta\left(T, x\left(T, 0, x^{0}, T, 0\right)\right.$, $\left.p\left(T, 0, x^{0}, T, 0\right), 0\right)=-x^{0} e^{-T}+\lambda$. Now, $\eta^{*}\left(T, 0, x^{0}, 0\right)=0$ gives $T={ }_{0}-\ln \lambda<$ 0 , which does not work. Now, both $\eta^{*}\left(b, 0, x^{0}, 0\right)>0$, and, for any $\eta^{*}\left(0,0, x^{0}, 0\right)>$ 0 , so the only useful proposal is $T\left(0, x^{0}, 0\right)=b$.

Remark 22 directly gives that this proposal $T\left(0, x^{0}, 0\right)=b$ is optimal, since $\eta^{*}\left(T, 0, x^{0}, 0\right) \geq 0$ for all $T$.

To use Theorem 8 , we need entities $x(t ; s, y, 0), p(t ; s, y, 0)$ and $T(s, y, 0)$ also for $(s, y) \neq\left(0, x^{0}\right)$. But since here $Q(0)=\left\{\left(s, x^{0} e^{-s}\right): s \in(0, b)\right\}$, we need only consider points $(s, y)$, for which $y$ is very close to $x^{0} e^{-s}$, and for such points $\eta^{*}(T, s, y, 0) \approx \eta^{*}\left(T, s, x^{0} e^{-s}, 0\right)=\eta^{*}\left(T, 0, x^{0}, 0\right)>0$, so $T(s, y, 0)=b$, and Theorem 8 then also gives optimality.

Finally, let us state necessary conditions for the present problem. (Again, normally they are satisfied, but exceptions exist.)

Theorem 9 (Necessary condition for free terminal time) Assume that $x^{*}(t, \omega), u^{*}(t, \omega), T(\omega)$ is an optimal triple. Then the necessary conditions of Theorem 1 are satisfied for $T$ replaced by $T\left(\tau_{0}, \ldots, \tau_{j}\right)$ (i.e. for any given $j$, the stated conditions are satisfied on $\left[0, T\left(\tau_{0}, \ldots, \tau_{j}\right)\right]$, moreover, a.s., [63] is satisfied for $T(s, y, j), \eta(T, x(T ; s, y, T, j), p(T ; s, y, T, j), j)$ replaced by $\left(T(\omega), \eta\left(T, x^{*}(T, \omega), p(T, \omega), j\right)\right.$ when $T(\omega) \in\left(\tau_{j}, \tau_{j+1}\right)$.

\section{The HJB-equation in optimal stopping}

An alternative solution tool is the HJB-equation. Assume for the moment that $[0, T]$ in $[57]$ is replaced by $[s, T]$, and, moreover that the maximum is 
found for all pairs $(x(t, \omega), u(t, \omega))$ where $x(.,$.$) starts at (s, y, j)$ and we allow $T$ to be subject to choice in $\left[\max \left\{s, T_{1}\right\}, T_{2}\right]$. The maximal value obtained is denoted $J^{*}(s, y, j)$, (the value will evidently depend on $(s, y, j)$ ). When the solution procedure above is used, in well behaved cases, $J(s, y, j)$ coincides $J^{*}(s, y, j)$. Let the set ("continuation region") $G^{*}(j)$ be defined by:

$$
G^{*}(j)=\left\{(s, y) \in\left[T_{1}, T_{2}\right] \times \mathbb{R}^{n}, J^{*}(s, y, j)>h_{0}(s, y)\right\}
$$

By definition of $G^{*}(j), J^{*}(s, y, j) \leq h_{0}(s, y)$ for $(s, y) \in G_{*}(j):=\left(\left[T_{1}, T_{2}\right] \times\right.$ $\left.\mathbb{R}^{n}\right) \backslash G^{*}(j)$. But, of course, the optimal value is never strictly smaller than $h_{0}(s, y)$, since we always have the possibility to stop immediately at $(s, y)$ and get $h_{0}(s, y)$. So, $J^{*}(s, y, j)=h_{0}(s, y)$ for $(s, y) \in G_{*}(j)$. Moreover, it is natural to call $G^{*}(j)$ the continuation region. We don't stop immediately at $(s, y)$ when we are in $G^{*}(j)$, since by continuing, we can get something better: $J^{*}(s, y, j)$ instead of $h_{0}(s, y)$.

Assume that $J^{*}(s, y, j)$ is continuous in $(s, y)$. Then, normally, for each $j, J^{*}(s, y, j)$ satisfies the conditions [70],[71] and [72] below:

$$
J^{*}(s, y, j)=h_{0}(s, y) \text { for }(s, y) \in \partial^{*} G^{*}(j):=\left\{(s, y) \in \partial G^{*}(j), s>T_{1}\right\}
$$

where $\partial G^{*}(j)$ denotes the boundary of $G^{*}(j)$.

At each point $(s, y) \in\left(\left[0, T_{1}\right) \times \mathbb{R}^{n}\right) \cup G^{*}(j)$ at which $J^{*}(s, y, j)$ is differentiable in $(s, y)$, the following equality, (HJB-equation) holds, for $J=J^{*}$.

$$
\begin{gathered}
0=J_{s}(s, y, j)+\sup _{u \in U}\left\{f_{0}(s, y, u, j)+J_{y}(s, y, j) f(s, y, u, j)\right\} \\
+\lambda(j+1)\left\{g_{0}(s, y, j+1)+J(s, y+g(s, y, j+1), j+1)-J(s, y, j)\right\}
\end{gathered}
$$

Moreover, the following inequality holds for all $(s, y) \in \partial G^{*}(j), s \in$ $\left(T_{1}, T_{2}\right)$ :

$$
\begin{array}{r}
0 \geq h_{0 t}(s, y)+\sup _{u \in U}\left\{f_{0}(s, y, u, j)+h_{0 x}(t, y) f(s, y, u, j)\right\} \\
+\lambda(j+1)\left\{g_{0}(s, y, j+1)+J(s, y+g(s, y), j+1)-h_{0}(s, y)\right\}
\end{array}
$$


The intuition behind [72] is as follows: Denote the expression on the righthand side when the sup sign is dropped by $\alpha(s, u, y)$. The entity $\alpha(s, y, u) d s$ gives the change in reward if instead of stopping immediately at $s$, we continue to $s+d s$, (see the next section below). Clearly, this must be $\leq 0$ for any control $u$.

Equality [71] is the HJB-equation of the problem. The problem now consists in solving the HJB-equation [71] for the unknown $J$, with boundary conditions [70], [72].

Also in the optimal stopping case, a sufficient condition is connected with the HJB- equation.

Theorem 10 (Sufficient condition for the HJB-equation) Assume that open sets $\hat{G}(j) \subset \mathbb{R}^{n+1}$ and functions $J(s, y, j)$, defined on $\operatorname{cl} Q(j)$ have been found, being $C^{1}$ and satisfying [71] in $\left.D(j):=Q(j) \cap \hat{G}(j)\right\}$ and satisfying $J(t, x, j)>h_{0}(t, x)$ for $(t, x)$ in $D(j), t \geq T_{1}$, and $Q(j) \cap\left(\left[0, T_{1}\right) \times \mathbb{R}^{n}\right) \subset \hat{G}(j)$.

Moreover, assume that for $(s, y) \in Q(j) \cap\left(\left[T_{1}, T_{2}\right] \times \mathbb{R}^{n}\right) \backslash \hat{G}(j), J(t, x, j)=$ $h_{0}(t, x)$, that [68] is satisfied here, and that $J(s, y, j)$ is $C^{0}$ on $\operatorname{cl} Q(j)$. Assume also that for each $(s, y) \in(\partial \hat{G}(j)) \cap Q(j), s \in\left(T_{1}, T_{2}\right)$, for all $u \in U$, the condition [67] holds, for a suitable $C^{1}$-extension of $\left.J(s, y, j)\right|_{\hat{G}(j)}$. Assume, furthermore that, for any admissible pair $x(t, \omega), T(\omega)$, for any $\omega$, for all $t,|J(t, x(t, \omega), j)| \leq \alpha_{x(. . .)}+\kappa_{x(. .)}|x(t, \omega)|$ for some positive constants $\alpha_{x(. .)}, \kappa_{x(. .)}$, and $t \rightarrow J\left(t, x\left(t, \omega^{j}\right), j\right), t \in\left(\tau_{j}, T(\omega)\right)$, is Lipschitz continuous in $t$ with Lipschitz rank $\beta_{x(., .)}\left(1+\kappa_{g}\right)^{j}$, for some constant $\beta_{x(., .)}$. Assume, finally, that there exists a control function $u^{*}(t, \omega)$, with corresponding solution $x^{*}(t, \omega)$, which yields the supremum in the HJB-equation [71], for $(s, y)=\left(s, x^{*}(s, \omega)\right) \in D(j)$. Then $\left(u^{*}(t, \omega), T^{*}(\omega)\right)$ is an optimal pair in the class of all pairs $u(t, \omega), T(\omega)$, where $T^{*}(\omega):=\inf \left\{T_{j}\left(\omega^{j}\right): T_{j}\left(\omega^{j}\right)<\tau_{j+1}\right\}$, $T_{j}\left(\omega^{j}\right)=\sup \left\{t>\tau_{j}:\left(s, x^{*}\left(s+, \omega^{j}\right)\right) \in D(j)\right.$ for $\left.s \in\left[\tau_{j}, t\right)\right\}, T_{j}\left(\omega^{j}\right)=\tau_{j}$ if the set is empty.

As said before, a standard method for solving the HJB-equation is to use the characteristic equations. Thus, note that the functions $J(s, y, j)$ defined in [64] automatically satisfies [71] for $(s, y) \in G_{j}$, provided enough differentiability is exhibited by the functions $x(t ; s, y, T, j), p(t ; s, y, T, j)$, and $T(s, y, j)$.

Example 9 Assume in Example 8 the possibility of an infinite number of jumps, all with intensity $\lambda$. The HJB-equation $0=J_{s}+J_{x}(-x)+\lambda J-\lambda J(x+$ 1) (as well as the adjoint equation) is now satisfied for a single function $J$ 
independent of $j$.

Let us do some preliminary calculations. The expected value $z(\sigma)$ of $x(\sigma)$, $\sigma \in(t, b]$ can be found as follows: Given $x(\sigma)$, the conditional expected value of $x(\sigma+d \sigma)$ is approximately equal to $(x(\sigma)+\dot{x}(\sigma) d \sigma)(1-\lambda d \sigma)+$ $(x(\sigma)+1) \lambda d \sigma$, the two terms arising, respectively, from the two events: no jump in $(\sigma, \sigma+d \sigma)$, and one jump in $(\sigma, \sigma+d \sigma)$. (For $d \sigma$ small the possibility of more than one jump may be discarded.) Then $\operatorname{Ex}(\sigma+d \sigma) \simeq$ $x(\sigma)(1-\lambda d \sigma)-x(\sigma) d \sigma(1-\lambda d \sigma)+(x(\sigma)+1) \lambda d \sigma \simeq x(\sigma)-x(\sigma) d \sigma+\lambda d \sigma$. Hence, $E[x(\sigma+d \sigma)-x(\sigma)] / d \sigma \simeq-x(\sigma)+\lambda$, (still conditional expectation). Hence, taking unconditional expectation on both sides gives $\dot{z}=-z(\sigma)+\lambda$. This differential equation has the solution $z(t)=\lambda+(y-\lambda) e^{-(t-s)}, \quad(z(s)=y)$. Does it pay to stop at $b$ rather than stopping at once? This requires $y<z(b)$, which yields $y\left(1-e^{-(b-s)}\right)<\lambda\left(1-e^{-(b-t)}\right)$, or $y<\lambda$. So if this inequality holds it pays to continue. (In fact in example 7 above, we saw that even if only one jump may occur, it pays to continue.) What is the optimal strategy if $y>\lambda$ ? We suspect that if $\mathrm{y}$ is not too large it may still pay to continue.

It is difficult to solve the HJB-equation (or the adjoint equation) in this case. So a guess is needed. Let us calculate the expected reward when starting in $(s, y)$ and waiting until the first jump occurs and then stopping at once, or if no jump occurs stopping at b. (Denote this policy $R^{*}$.) The expected reward is evidently

$$
\begin{gathered}
\int_{s}^{b}\left(y e^{s-\rho}+1\right) \lambda e^{\lambda(s-\rho)} d \rho+\int_{b}^{\infty} y e^{s-b} \lambda e^{\lambda(s-\rho)} d \rho \\
=\eta(s, y):=y \lambda /(1+\lambda)\left(1-e^{(1+\lambda)(s-b)}\right)+1-e^{\lambda(s-b)}+y e^{(1+\lambda)(s-b)}
\end{gathered}
$$

Note that $\eta(b, y) \geq \lambda$, when $y \geq \lambda$. Note also that $\partial \eta(s, y) / \partial s=$ $y e^{(1+\lambda)(s-b)}-\lambda e^{\lambda(s-b)}$ is negative when $y=\lambda, s<b$. Thus for $y>\lambda$, the function $t \rightarrow \eta(t, \lambda)$ stays above $\lambda$ when going backwards. Thus the solution $x_{*}(s)$ of $y=\eta(t, y)$ satisfies $x_{*}(t)>\lambda$. The formula is

$$
x_{*}(s)=(1+\lambda)\left(1-e^{\lambda(s-b)}\right) /\left(1-e^{(1+\lambda)(s-b)}\right)
$$

which evidently is $\leq(1+\lambda)$.

We have seen that $(1+\lambda) \geq x_{*}(s) \geq \lambda$. Compared to the above strategy, $\left(R^{*}\right)$, it pays to stop if $y>x_{*}(s)$. Note also that if $y<\lambda$ then it pays to continue even if only one jump can occur as seen above. (And perhaps we don't stop immediately after that jump.) At such $y$ we surely know what to 
do. So from now on we only consider $y \geq \lambda$.

Note that then $y+1 \geq \lambda+1 \geq x_{*}(s)$. If we propose the set $\left\{(s, y): y \geq x_{*}(s)\right\}$ to be the "stopping region", then we see that if $\lambda \leq y \leq x_{*}(s)$, a jump at $(s, y)$ brings $y+1$ into this stopping set. Thus if we now, put $J(s, y)=\eta(s, y)$ in the continuation region $\left\{(s, y): y<x_{*}(s)\right\}$, while $J(s, y)=y$ elsewhere, theoretical results tell us that $J(s, y)$ satisfies the HJB equation, for $(s, y)$ in $G_{\lambda}:=\left\{(s, y): s<b, \lambda<y<x_{*}(t)\right\}$. To explain intuitively the satisfaction of the HJB-equation, simply note that $J(t, x)$ as here defined is the optimal value function in a very restricted problem: That one in which we are given no choices, and in which we get the reward $y+1$, when we jump from $(s, y) \in G_{\lambda}$ to $(s, y+1)$. However, that $J(s, y)$ satisfies the HJB-equation in $G_{\lambda}$ can be directly tested by insertion into the equation. We also check that the correct inequality [68] is satisfied by the function $h_{0}(s, y)=y$ outside the continuation region. Using sufficiency results (Theorem 10), we can conclude that the optimal policy has been found: Not only for $y<\lambda$, but also at points $(s, y) \in G_{\lambda}$, we continue. The continuation region in fact is $G:=\left\{(s, y): s<b, y<x_{*}(s)\right\}$. (We can in principle construct solutions of the HJB equation also for $(s, y) \in G,(s, y) \notin G_{\lambda}$, by successively constructing solutions in $\left\{(s, y): s<b, x_{*}(s)-2 \leq y<x_{*}(s)-1,\{(s, y): s<\right.$ $b, x_{*}(s)-3 \leq y<x_{*}(s)-2, \ldots$, since we always jump from a given set in this sequence to the preceding one in this sequence, on which we have already constructed the solution. But we don't need these solutions.)

Remark 23 End constraints Assume in problem [9], [10], [57], with $T$ subject to choice in $\left[T_{1}, T_{2}\right]$, that the following terminal conditions are introduced: A.s.,

$$
\begin{gathered}
x_{i}(T(\omega), \omega)=\hat{x}_{i}, i=1, \ldots, n^{\prime}, \\
x_{i}(T(\omega), \omega) \geq \hat{x}_{i}, i=n^{\prime}+1, \ldots, n^{\prime \prime} .
\end{gathered}
$$

Then Theorems 8 and 10 also hold in the case of end constraints, provided the following changes are made: In case of Theorem 10, drop the assumption that $J(s, y, j)$ is $C^{0}$ on $\operatorname{cl} Q(j)$, (but not the $C^{1}$-assumption) on $J(s, y, j)$ ), and add the condition that

$$
\lim _{t \uparrow T^{*}(\omega)} J\left(t, x^{*}(t, \omega), j\right)=h_{0}\left(T^{*}(\omega), x^{*}\left(T^{*}(\omega)\right),\right.
$$

if $\tau_{j} \leq T^{*}(\omega)<\tau_{j+1}$, and that, for any admissible $x(.,$.$) ,$

$$
\text { if }\left(s, x\left(s, \omega^{j}\right)\right) \in \partial \hat{G}(j), s>T_{1} \text {, then } \lim _{t \rightarrow s} J\left(t, x\left(t, \omega^{j}\right), j\right)=h_{0}(s, y) \text {. }
$$


In case of Theorem 8 , drop [68**, and add [74b] for $\hat{G}(j)$ replaced by $G_{j}$, with $p(T)=\left[h_{0 x}(T, x)\right]_{x=x(T ; s, y, T, j)}$ replaced by [43] for $T=T(s, y, j)$, $\lambda_{i}(k, s, y, j)$ continuous for $i=n^{\prime}+1, \ldots, n^{\prime \prime}$. Finally, replace the boundedness conditions on $M(s, y, j):=p(s ; s, y+g(s, y, j), j+1)$ and on $p_{y}(s, s, y+$ $g / s, y, j), j+1)$ by the local boundedness conditions that

$\sup _{t \in\left[s, T\left(s, y, \omega^{j}\right)\right)}|M(t, x(t ; s, y), j)|, \sup _{t \in\left[s, T\left(s, y, \omega^{j}\right)\right)}\left|M_{x}(t, x(t ; s, y), j)\right|$ and $\sup _{t \in[s, T(s, y, j))}\left|M_{x x}\left(t, x(t ; s, y), \omega^{j}\right)\right|$ are locally bounded on $D^{0}\left(\omega^{j}\right)$.

Furthermore, normally, Theorem 9 holds with the modification that the transversality conditions must be those appearing in Theorem 4, changed by replacing $T$ by $T^{*}(\omega)$. (Again only the cases [42] are considered, and [45] is assumed, for $T=T^{*}(\omega)$.)

\section{Proofs of a selection of the above results}

Proofs are given for sufficient conditions (verification results) based on the HJB-equation and characteristic solutions, as well as for the necessity of the HJB-equation. Certain details are treated with less than full rigor. To simplify the notation, we shall consider the special case where $f_{0} \equiv 0$ and $g_{0} \equiv 0$. The general case is treated by applying the "special case" to a redefined problem where an auxiliary additional state $x^{0}$ is introduced governed by

$$
\begin{gathered}
\dot{x}^{0}=f_{0}(t, x, u, j), x^{0}(0)=0, u \in U \\
x^{0}\left(\tau_{j}+\right)-x^{0}\left(\tau_{j}-\right)=g_{0}\left(\tau_{j}, x\left(\tau_{j}-\right), V_{j}, w_{j}, j\right),
\end{gathered}
$$

and where

$$
E\left[x^{0}(T-)+h_{0}(T, x(T-))\right]
$$

is maximized. We shall only consider the case where $W_{j}=W$, the modifications needed if these sets depend on $j$ being obvious.

In this "special problem", certain generalizations, as mentioned in various Remarks above are introduced. It is assumed that a function $\lambda=\lambda(t, x, u, \omega)$, is given, $\lambda(t, x, u, \omega)$ being nonanticipating in $(t, \omega), \omega=\left(\tau_{0}, V_{0}, \tau_{1}, V_{1}, \ldots\right)$. For any $k$, the function $\lambda(t, x, u, \omega), t \geq \tau_{k}$, gives the intensity of the next jump $\tau_{k+1}$ in $\left[\tau_{k}, \infty\right)$. The function $\lambda(t, x, u, \omega)$ is piecewise and left-continuous in $t$, piecewise and right-continuous in each $\tau_{k}, V_{k}, k>0$, and continuous in $(x, u)$, uniformly in $t, \omega$. Moreover, $0 \leq \lambda(., ., .,) \leq$.$K , for some K>0$. Furthermore, for any $k$, the cumulative probability distribution of $V_{k}$ is given by $\pi(v)=\pi\left(v ; \tau_{k} ; x, \omega\right)$, where $\pi(v ; t ; x, \omega)$ is nonanticipating as a function 
of $(t, \omega)$, so $\pi(v ; t ; x, u, \omega)$ depends only on $\tau_{i}, V_{i}$, such that $\tau_{i}<t$. Moreover, $\pi(v ; t ; x, \omega)$ is nondecreasing in $v$, (thus having one-sided limits in $v$ ), in fact, for simplicity we assume piecewise continuity in $v$, and continuous differentiability, except at jump points. Furthermore, $\pi(v ; t ; x, \omega)$ is piecewise and left-continuous in $t$ uniformly in $\omega$, continuous in $x$, uniformly in $t, \omega$, and piecewise and right-continuous in each $\tau_{i}, V_{i}, i<k$. The problem takes the form:

$$
\max E h_{0}(T, x(T-, \omega)),
$$

subject to

$$
\dot{x}=f(t, x, u(t, \omega), \omega), x(0)=x^{0}, u \in U
$$

the hard terminal conditions [39], $w(t, v, \omega) \in W$ and

$$
x\left(\tau_{k}+, \omega\right)-x\left(\tau_{k}-, \omega\right)=g\left(\tau_{k}, x\left(\tau_{k}-, \omega\right), V_{k}, w\left(\tau_{k}, V_{k}, \omega\right), \omega\right) .
$$

Now, $f(t, x, u, \omega)$ and $g(t, x, v, w, \omega)$ depend on $\omega$, they are nonanticipating in $(t, \omega)$, (they depend only on $\tau_{i}, V_{i}$, for $i$ such that $\tau_{i}<t$ ). The control parameter $w(t, v, \omega) \in W$ determines the jump size at any $t=\tau_{k}, w(t, v, \omega)$ is nonanticipating, (only dependent on $\left.\left(\tau_{i}, V_{i}\right), \tau_{i}<t\right)$, and $w(t, v, \omega)$ is piecewise and left-continuous in $t$ and continuous in $V$, and piecewise and right-continuous in each $\tau_{i}, V_{i}, \tau_{i}<t$. Moreover, the controls $u(t, \omega) \in U$ are nonanticipating, piecewise and right-continuous in $t$, piecewise and left-continuous in each $\tau_{i}, V_{i}, \tau_{i}<t$. Such controls $w(t, v, \omega)$ and $u(t, \omega)$ are called admissible. It is assumed that $h_{0}$ is $C^{1}$, that $f$ and $f_{x}$, are uniformly continuous in $(x, u)$ uniformly in $t, \omega$, piecewise and left-continuous in $t$ uniformly in $\omega$, and piecewise and right-continuous in each $\tau_{i}$ and $V_{i}, \tau_{i}<t$. Furthermore, $g(t, x, v, w, \omega)$ and $g_{x}(t, x, v, w, \omega)$ are continuous in $(x, v, w)$ uniformly in $t, \omega$, piecewise and left-continuous in $t$ uniformly in $\omega$ and piecewise and rightcontinuous in each $\tau_{i}$, and in each component $V_{i}, \tau_{i}<t$. Moreover, $h_{0}, f$ and $g$ are Lipschitz continuous in $x$ of ranks $\kappa_{h_{0}}, \kappa_{f}$ and $\kappa_{g}$ and for some constants $\alpha_{f}$ and $\alpha_{g},|f(t, x, u, \omega)| \leq \alpha_{f}+\kappa_{f}|x|,|g(t, x, v, w, \omega)| \leq \alpha_{g}+\kappa_{g}|x|$ for all $t, x, u, v, w, \omega$. We allow $N=\infty$. First, the fixed horizon case, is studied, so until further notice, $T$ is fixed $>0$ and then we write $h_{0}(T, x)=h_{0}(x)$.

Let $\omega_{j}=\left(\tau_{j+1}, V_{j+1}, \tau_{j+2}, V_{j+2}, \ldots\right)$. We say that $\left(s, y, \omega^{j}\right)$ is a starting point for a solution $x\left(t ; s, y, \omega_{j}\right), t \geq s$, if exactly $j$ jumps have occurred at $\tau_{1}<$ $\ldots<\tau_{j}<s$, and $x\left(s-; s, y, \omega_{j}\right)=y$. Let $\left(s, y, \omega^{j}\right)$ be a given triple, and imagine that in the above setting, $\left(0, x^{0}, \omega^{0}\right)\left(\omega^{0}\right.$ as before indicating zero jumps) is replaced by $\left(s, y, \omega^{j}\right)$. Then $\omega$ will be replaced by $\omega_{j}$, we write $u\left(t, \omega_{j}\right)$ and $w\left(t, v, \omega_{j}\right)$ and the solution starting in $\left(s, y, \omega^{j}\right)$ corresponding 
to these controls is written $x^{u, w}\left(t ; s, y, \omega_{j}\right)$. Define

$$
J^{*}\left(s, y, \omega^{j}\right)=\sup _{u, w} E\left[h_{0}\left(x^{u, w}\left(T-, \omega_{j}\right)\right) \mid s, y, \omega^{j}\right],
$$

the supremum taken over all admissible $u\left(., \omega_{j}\right), w\left(., \omega_{j}\right)$ which has a corresponding solution $x^{u, w}\left(., s, y, \omega_{j}\right)$ starting in $\left(s, y, \omega^{j}\right)$ and satisfying the terminal conditions. (Such a solution is called $\left(s, y, \omega^{j}\right)$-admissible.)

Assume that for each given $\omega^{j}, Q\left(\omega^{j}\right)$ consist of all points $(s, y), s \in\left[\tau_{j}, T\right)$ that can be reached by some $\left(0, x^{0}, \omega^{0}\right)$-admissible solution $x(t, \omega)$ after $j$ jumps, for the given "jump parameters" in $\omega^{j}$, (i.e. $(s, y)=(s, x(s+, \omega))$. Below, we shall make use of the HJB - equation, that reads: For all $\omega^{j}$, for all $(s, y) \in Q\left(\omega^{j}\right), s>\tau_{j}$,

$$
\begin{gathered}
0=J_{s}\left(s, y, \omega^{j}\right)+\sup _{u \in U}\left\{J_{y}\left(s, y, \omega^{j}\right) f\left(s, y, u, \omega^{j}\right)+\right. \\
\lambda\left(s, y, u, \omega^{j}\right) E\left[\sup _{w \in \hat{W}} J\left(s, y+g\left(s, y, V, w, \omega^{j}\right),\left(\omega^{j}, s, V\right)\right) \mid s, y, \omega^{j}\right]- \\
\left.\lambda\left(s, y, u, \omega^{j}\right) J\left(s, y, \omega^{j}\right)\right\}
\end{gathered}
$$

where $\hat{W}:=\hat{W}\left(s, y, V, \omega^{j}\right):=\left\{w \in W: y+g\left(s, y, V, w, \omega^{j}\right) \in Q\left(\omega^{j}, s, V\right)\right\}$, and where the expectation is calculated by means of $\pi\left(v ; s ; y, \omega^{j}\right)$. Below, control functions $u^{*}(t, \omega)$ and $w^{*}(t, \omega)$, with a corresponding solution $x^{*}(t, \omega)$ appear. We shall make use of the two properties:

$w^{*}\left(s, V, \omega^{j}\right)$ yields the supremum over $w$ in [82], for $y=x^{*}\left(s-, \omega^{j}\right)$, [83]

$u^{*}\left(s, \omega^{j}\right)$ yields the supremum over $u$ in [82] for $y=x^{*}\left(s-, \omega^{j}\right)$.

We shall also make use of the following boundary condition: A.s., for $\omega$ such that $\tau_{j}<T \leq \tau_{j+1}$,

$\lim _{t \rightarrow T} E J\left(t, x(t-, \omega), \omega^{j}\right)=E h_{0}(x(T-, \omega))$ for any admissible $x(t, \omega)$, $[85]$

We shall prove the following theorem: (For nonanticipating functions $\psi(s, \omega)$ recall that we have $\psi(s, \omega)=\psi\left(s, \omega^{j}\right)$ when $s \in\left[\tau_{j}, \tau_{j+1}\right)$.)

Theorem 11 Sufficient condition for the HJB-equation Assume that $J\left(s, y, \omega^{j}\right)$ is a function defined on $Q\left(\omega^{j}\right)$ and define $J(s, y, \omega)=J\left(s, y, \omega^{j}\right)$ if $s \in\left[\tau_{j}, \tau_{j+1}\right)$, letting $Q(\omega):=\left\{(s, y): s \in\left[\tau_{j}, \tau_{j+1}\right)\right.$ for some $j$ and $\left.(s, y) \in Q\left(\omega^{j}\right)\right\}$ be the domain of definition of $J(s, y, \omega)$.. For $s \in\left(\tau_{j}, \tau_{j+1}\right)$ assume that $J(s, y, \omega)=J\left(s, y, \omega^{j}\right)$ is extendable to some open sets larger than 
$\left\{(s, y): Q\left(\omega^{j}\right), s>\tau_{j}\right\}$, being $C^{1}$ in $(s, y)$ here, satisfying the HJB-equation [82] (for derivatives stemming from the $C^{1}$-extension), with $J, J_{s}$, and $J_{y}$ being, separately, piecewise and right-continuous in each $\tau_{i}$, each component of each $V_{i}$. Assume that, for any admissible solution $x(t, \omega), J(s, y, \omega)$ satisfies the boundary condition [85], as well as the conditions that $|J(t, x(t, \omega), \omega)| \leq$ $\alpha_{J}+\kappa_{J}|x(t, \omega)|$, and that $s \rightarrow J(s, x(s+, \omega), \omega)$ for $s \in\left[\tau_{j}, \tau_{j+1}\right)$ is Lipschitz continuous with rank $\hat{\kappa}_{J}\left(1+\kappa_{g}\right)^{j}$, uniformly in $\omega$, for some constants $\alpha_{J}, \kappa_{j}, \hat{\kappa}_{J}$. (The constants $\alpha_{J}, \kappa_{J}$, and $\hat{\kappa}_{J}$ are perhaps dependent on $x(.,$.$) .)$ Assume that there exists an admissible triple $\left(x^{*}(., \omega), u^{*}(., \omega), w^{*}(., V, \omega)\right)$ satisfying [83] and [84]. Then $\left(u^{*}(t, \omega), w^{*}(t, V, \omega)\right)$ is optimal in the problem [78]-[80],[39].

The condition [85] is automatically satisfied if, for each $\omega$ such that $\tau_{j}<$ $T<\tau_{j+1}, J(s, y, \omega)$ can be extended continuously to $Q\left(\omega^{j}\right) \cup\left(\{T\} \times \mathbb{R}^{n}\right) \cap \mathrm{cl}$ $\left.Q\left(\omega^{j}\right)\right)$, and $J(T, x, \omega)=h_{0}(x)$ holds. In the free end case, very often functions $J$ can be found, for which these two conditions hold.

Remark 24 (Weakened differentiability conditions) In Theorem 11 it suffices that for each admissible $x(., \omega), J\left(s, y, \omega^{j}\right)$ is $C^{1}$ in a neighborhood of each point $(s, x(s, \omega)), s \in\left(\tau_{j}, \tau_{j+1}\right)$, except at a countable number of point $\sigma_{k}^{j}\left(\omega^{j}\right),\left(\sigma_{k}^{j}\left(\omega^{j}\right)\right.$ right-continuous in each $\left.\tau_{i}, V_{i}\right)$.

Theorem 12 (Necessary condition for the HJB-equation) Assume that the sets $Q^{\prime}\left(\omega^{j}\right):=\left\{(s, y) \in Q\left(\omega^{j}\right): s>\tau_{j}\right\}$ are open, and that $(s, y) \rightarrow$ $J^{*}\left(s, y, \omega^{j}\right), s \in\left(\tau_{j}, \tau_{j+1}\right)$, is $C^{1}$ in $Q^{\prime}\left(\omega^{j}\right)$. Let $x^{*}(., \omega), u^{*}(., \omega), w^{*}(., V, \omega)$ be an optimal triple in the problem [78]-[80], [39]. Assume moreover, that for each $j$, each $\omega^{j},(s, y) \in Q^{\prime}\left(\omega^{j}\right)$, there exist optimal pairs $u^{*}\left(t, \omega_{j} ; s, y, \omega^{j}\right)$, $w^{*}\left(t, V, \omega_{j} ; s, y, \omega^{j}\right)$ with a corresponding solution $x\left(t, \omega_{j} ; s, y, \omega^{j}\right)$ starting at $\left(s, y, \omega^{j}\right)$, with the property that for any admissible solution $x(., \omega)$, $u^{*}\left(t, \omega_{j} ; s, x\left(s, \omega^{j}\right), \omega^{j}\right), w^{*}\left(t, V, \omega_{j} ; s, x\left(s, \omega^{j}\right), \omega^{j}\right)$, as functions of $\left(s, \omega^{j}, \omega_{j}\right)$, satisfy the same continuity conditions as $x^{*}(s, \omega), u^{*}(s, \omega), w^{*}(., V, \omega)$. Then $J^{*}\left(s, y, \omega^{j}\right)$ satisfies [82],[84] and

$$
\begin{aligned}
& {\left[\lambda\left[\left(s, y, u, \omega^{j}\right) E\left[\sup _{w \in \hat{W}} J\left(s, y+g\left(s, y, V, w, \omega^{j}\right),\left(\omega^{j}, s, V\right)\right) \mid s, y, \omega^{j}\right]\right]_{y=x^{*}\left(s-, \omega^{j}\right)} \leq\right.} \\
& \quad\left[\lambda\left[\left(s, y, u, \omega^{j}\right) E\left[J\left(s, y+g\left(s, y, V, w^{*}\left(s, V, \omega^{j}\right), \omega^{j}\right),\left(\omega^{j}, s, V\right)\right) \mid s, y, \omega^{j}\right]\right]_{y=x^{*}\left(s-, \omega^{j}\right)}\right.
\end{aligned}
$$

Of course, $J^{*}\left(t, x\left(t-, \omega^{j}\right), \omega^{j}\right) \geq E\left[h_{0}(x(T-, \omega)) \mid t, x\left(t-, \omega^{j}\right), \omega^{j}\right]$, with equality a.s. if $x(.,)=.x^{*}(.,$.$) . Now, when t$ is close to $T$, there is only a small probability that additional jumps occur in $[t, T]$, so when $t \rightarrow T$, then 
$E\left[h_{0}(x(T-, \omega)) \mid t, x\left(t-, \omega^{j}\right), \omega^{j}\right] \rightarrow h_{0}(x(T-, \omega))$. Hence, [85] evidently holds for $J=J^{*}$.

Remark 25 In Theorem 12, in fact, [82], [84], and [86] hold, even when $u^{*}\left(t, \omega_{j} ; s, y, \omega^{j}\right), w^{*}\left(t, V, \omega ; s, y, \omega^{j}\right)$ do not exist, and for [82] to hold, we can even drop the existence of $u^{*}(t, \omega), w^{*}(t, V, \omega)$.

For use in the formulation of a sufficient condition based on characteristic triples, we shall now state a transversality condition needed in the end constrained case. Thus in Theorem 13 below, in the end constrained case, $g$ is assumed to depend only on $t, x, j, V_{j}$ and the last jump time $\tau_{j}$ before $t$, so $g\left(t, x, V_{j}, w, \omega^{j}\right)=g\left(t, x, V_{j}, \tau_{j}, j\right)$. Define $\hat{g}^{0, j}(x)=x, \hat{g}^{1, j}\left(x, V_{j+1}\right)=$ $\hat{g}^{0, j}(x)+g\left(T, \hat{g}^{0, j}(x), V_{j+1}, T-, j+1\right), \ldots, \hat{g}^{k, j}\left(x, V_{j+1}, \ldots, V_{k}\right)=$ $\hat{g}^{k-1, j}\left(x, V_{j+1}, . ., V_{j+k-1}\right)+g\left(T, \hat{g}^{k-1, j}\left(x, V_{j+1}, . ., V_{j+k-1}, V_{k}, T-, k\right)\right.$. Next, define, $g^{k, j}\left(x, \omega^{j}\right)=$

$\operatorname{essinf}_{V_{j+1}, \ldots, V_{k}} \hat{g}^{k, j}\left(x, V_{j+1}, \ldots, V_{j+k}\right)$.

For any $\omega^{j}$, such that $T>\tau_{j}$, there exist multipliers $\Lambda_{i}\left(k, s, y, \omega^{j}\right), i=$ $1 \ldots, n^{\prime \prime}, k=0, \ldots, N-j$, with $\Lambda\left(k, s, y, \omega^{j}\right)=$ $\left(\Lambda_{1}(k, s, y, \omega), \ldots, \Lambda_{n^{\prime \prime}}(k, s, y, \omega), 0 \ldots, 0\right) \in \mathbb{R}^{n}$, such that

$p\left(T ; s, y, \omega^{j}\right)=h_{0 x}\left(x\left(T ; s, y, \omega^{j}\right)\right)+\sum_{0 \leq k \leq N-j} \Lambda\left(k, s, y, \omega^{j}\right) g_{x}^{k, j}\left(x\left(T ; s, y, \omega^{j}\right), \omega^{j}\right)$,
$\Lambda_{i}\left(k, s, y, \omega^{j}\right) \geq 0, \Lambda_{i}\left(k, s, y, \omega^{j}\right)=0$ if $\left(g^{k, j}\left(x\left(T ; s, y, \omega^{j}\right), \omega^{j}\right)\right)_{i}>\hat{x}_{i}, i=n^{\prime}+1, \ldots, n^{\prime \prime}$.
In case [42a], $\Lambda\left(k, s, y, \omega^{j}\right)=0, k>0$.

(Actually, we here need that [42a] holds for all $\omega^{j}$.) In the free end case, [86*] reduces to $p\left(T ; s, y, \omega^{j}\right)=h_{0 x}\left(x\left(T ; s, y, \omega^{j}\right)\right)$, in which case $g$ can have the general form appearing in $\left[86^{* *}\right]$ below.

In the next theorem, we assume that $\lambda$ is independent of $x$ and $u$, and $\pi$ is independent of $x$. Define $M\left(t, x, \omega^{j}\right):=E\left[\hat{M}\left(t, x, \omega^{j}, V_{j+1}\right) \mid t, \omega^{j}\right]$, where

$$
\hat{M}\left(t, x, \omega^{j}, V_{j+1}\right):=\sup _{w} J\left(t, x+g\left(t, x, V_{j+1}, w, \omega^{j}\right), \omega^{j}, t, V_{j+1}\right)
$$




\section{Theorem 13 (Sufficiency based on a field of characteristic solutions)}

Consider the problem [78]-[80],[39]. Assume that $f$ is $C^{0} \times C^{2}$ in $t$ and $(x, u)$, that $h_{0}$ is $C^{2}$, that $g$ is $C^{0} \times C^{2}$ and that $\lambda(t, \omega)$ is continuous in $t$. Assume that $\hat{u}\left(t, x, p, \omega^{j}\right)$ yields maximum of $H\left(t, x, u, p, \omega^{j}\right):=p f\left(t, x, u, \omega^{j}\right)$, for $(t, x, p)$ in a given open set $Q^{*}\left(\omega^{j}\right)$. For $s \in\left(\tau_{j}, \tau_{j+1}\right]$, assume that a collection of open sets $Q^{0}\left(\omega^{j}\right)$ in $\mathbb{R}^{n+1}$, containing $Q\left(\omega^{j}\right) \cap\left(\tau_{j}, T\right) \times \mathbb{R}^{n}$, and a collection of solutions $x\left(t ; s, y, \omega^{j}\right), p\left(t ; s, y, \omega^{j}\right), z\left(t ; s, y, \omega^{j}\right)$, continuous in $t \in[s, T]$, have been found, satisfying $\dot{x}=f\left(t, x, \hat{u}\left(t, x, p, \omega^{j}\right), \omega^{j}\right)$, $\dot{p}=-p f_{x}\left(t, x, \hat{u}\left(t, x, p, \omega^{j}\right), \omega^{j}\right)-\lambda\left(t, \omega^{j}\right) M_{x}\left(t, x, \omega^{j}\right)+\lambda\left(t, \omega^{j}\right) p$, $\dot{z}=\lambda\left(t, \omega^{j}\right) z-\lambda\left(t, \omega^{j}\right) M\left(t, x, \omega^{j}\right), z\left(T ; s, y, \omega^{j}\right)=h_{0}\left(x\left(T ; s, y, \omega^{j}\right)\right), x\left(t ; s, y, \omega^{j}\right)$ starting in $\left(s, y, \omega^{j}\right)$, for any $j$, any $\omega^{j}$, for any $(s, y) \in Q^{0}\left(\omega^{j}\right) \cup Q\left(\omega^{j}\right)$. These solutions are assumed to be, separately, piecewise and right-continuous in each $\tau_{i}$, and in each component of each $V_{i}$. Define $J\left(s, y, \omega^{j}\right)=z\left(s ; s, y, \omega^{j}\right)$, and let $J(s, y, \omega)=J\left(s, y, \omega^{j}\right)$ if $s \in\left(\tau_{j}, \tau_{j+1}\right],(s, y) \in Q\left(\omega^{j}\right)$. It is assumed that $x\left(t ; s, y, \omega^{j}\right), p\left(t ; s, y, \omega^{j}\right)$ satisfies the terminal conditions [39] and the transversality conditions $\left[86^{*}\right]$ with $\Lambda_{i}\left(k, s, y, \omega^{j}\right)$ continuous for $i=n^{\prime}+$ $1, \ldots, n^{\prime \prime}$. Assume that $\hat{w}\left(t, x, v, \omega^{j}\right)$ gives maximum of $J\left(t, x+g\left(t, x, v, w, \omega^{j}\right), \omega^{j}, t, v\right)$ for $(t, x) \in Q^{0}\left(\omega^{j}\right)$, and that the maximal value is a bounded function of $v$. Furthermore, assume that $(s, y) \in Q^{0}\left(\omega^{j}\right) \Rightarrow\left(s, y+g\left(s, y, \omega^{j}, s, V_{j+1}\right) \in\right.$ $Q^{0}\left(\omega^{j}, s, V_{j+1}\right)$. Moreover, assume that, for any $j$, any $\omega^{j},(s, y) \rightarrow\left(x\left(t ; s, y, \omega^{j}\right)\right.$, $\left.p\left(t ; s, y, \omega^{j}\right)\right)$ is $C^{1}$ for $(s, y) \in Q^{0}\left(\omega^{j}\right), s>\tau_{j}$, for any $t \in[s, T]$. Assume that, for all $(s, y) \in Q^{0}\left(\omega^{j}\right),\left(t, x\left(t ; s, y, \omega^{j}\right), p\left(t ; s, y, \omega^{j}\right)\right)$ belongs to $Q^{*}\left(\omega^{j}\right)$, for all $t \in[s, T]$ and that $\left(t, x\left(t ; s, y, \omega^{j}\right)\right)$ belongs to $Q^{0}\left(\omega^{j}\right)$ for all $t \in(s, T)$. Furthermore, assume that $\left(x\left(. ; s, y, \omega^{j}\right), p\left(. ; s, y, \omega^{j}\right)\right)$ satisfies the following "consistency condition":

$$
\begin{array}{r}
\left(x\left(t ; s^{\prime}, x\left(s^{\prime} ; s, y, \omega^{j}\right), \omega^{j}\right), p\left(t ; s^{\prime}, x\left(s^{\prime} ; s, y, \omega^{j}\right), \omega^{j}\right)\right)= \\
\left(x\left(t ; s, y, \omega^{j}\right), p\left(t ; s, y, \omega^{j}\right)\right), \text { for all }(s, y) \in Q\left(\omega^{j}\right), s<s^{\prime}<t .
\end{array}
$$

Moreover, assume that $\hat{H}\left(s, x, p, \omega^{j}\right)$ is $C^{0} \times C^{2} \times C^{2}$ in $Q^{*}\left(\omega^{j}\right)$, that $\hat{u}\left(s, x, p, \omega^{j}\right)$ is $C^{0} \times C^{1} \times C^{1}$ here, that $M\left(t, x, \omega^{j}\right)$ is $C^{0} \times C^{2}$ on $Q^{0}\left(\omega^{j}\right)$ and that $\sup _{t \in[s, T)}\left|M\left(t, x\left(t ; s, y, \omega^{j}\right), \omega^{j}\right)\right|, \sup _{t \in[s, T)}\left|M_{x}\left(t, x\left(t ; s, y, \omega^{j}\right), \omega^{j}\right)\right|$, $\sup _{t \in[s, T)}\left|M_{x x}\left(t, x\left(t ; s, y, \omega^{j}\right), \omega^{j}\right)\right|$ are locally bounded in $(s, y) \in Q^{0}\left(\omega^{j}\right)$. Assume that $J(s, y, \omega)$ satisfies [85], and for each admissible $x(., \omega)$, that $|J(t, x(t, \omega), \omega)| \leq \alpha_{J}+\kappa_{J}|x(t, \omega)|$ and that $s \rightarrow J(s, x(s+, \omega), \omega)$ is Lipschitz continuous in $\left(\tau_{j}, \tau_{j+1}\right)$, with constant $\hat{\kappa}_{J}\left(1+\kappa_{g}\right)^{j}, \alpha_{J}, \kappa_{J}, \hat{\kappa}_{J}$ perhaps dependent on $x(., \omega)$. Assume also that $J, J_{s}$, and $J_{y}$ are, separately, piecewise and right-continuous in each $\tau_{i}$, and each component of each $V_{i}$. Define $x(t, \omega)=x\left(t, 0, x^{0}, \omega^{0}\right)$, for $t \in\left[0, \tau_{1}\right]$, and by induction, generally, $x(t, \omega)=$ $x\left(t, \tau_{k}, x\left(\tau_{k}, \omega\right)+g\left(\tau_{k}, x\left(\tau_{k}, \omega\right), v_{k}, \hat{w}\left(\tau_{k}, x\left(\tau_{k}, \omega\right), v_{k}, \omega\right), \omega\right), \omega^{k}\right)$, for $t \in\left(\tau_{k}, \tau_{k+1}\right]$. 
Assume that $w(t, v, \omega):=\hat{w}\left(t, x(t ; \omega), v, \omega^{j}\right), t \in\left(\tau_{j}, \tau_{j+1}\right]$, satisfies the standard requirements on such controls described above. Assume, finally that $g^{k, j}\left(x, \omega^{j}\right)$ is $C^{2}$ in $x$, for all $k, j$. Define $u(t, \omega):=\hat{u}\left(t, x(t ; \omega), p(t ; \omega), \omega^{j}\right), t \in$ $\left(\tau_{j}, \tau_{j+1}\right]$. Then $u(t, \omega), w(t, v, \omega)$ make up an optimal pair.

Proofs of Theorems 11, 12, 13 We need a differential formula (infinitesimal generator) for piecewise deterministic equations. Let $x\left(t ; a, y, \omega_{j}\right), u\left(t, \omega_{j}\right)$, $w\left(t, v, \omega_{j}\right)$ be any nonanticipating triple satisfying [79],[80], starting in $\left(a, y, \omega^{j}\right)$ and write $\lambda\left(t, \omega_{j}\right):=\lambda\left(t, x\left(t ; a, y, \omega_{j}\right), u\left(t, \omega_{j}\right), \omega\right), \pi\left(v ; t, \omega_{j}\right):=\pi\left(v ; t, x\left(t ; a, y, \omega_{j}\right), \omega_{j}\right)$.

Lemma 1 Let $a, y, \omega^{j}$ be given entities, $a \in(0, T)$, and let $\phi(s, x, \omega)$ be a given function, such that $\phi(s, x, \omega), \phi_{s}(s, x, \omega)$ and $\phi_{x}(s, x, \omega)$, for each $\omega$, are continuous in $(s, x), s \in\left(\tau_{k}, \tau_{k+1}\right)$, nonanticipating in $(s, \omega)$, and, for each $(s, x)$, piecewise and right-continuous in each $\tau_{i}<s$, and in each component of each $V_{i}$. Let $x\left(s ; \omega_{j}\right):=x\left(. ; a, y, \omega_{j}\right)$ be any nonanticipating solution starting in $\left(a, y, \omega^{j}\right)$, corresponding to any given controls $u\left(t, \omega_{j}\right), w\left(t, v, \omega_{j}\right)$, $\left(\tau_{j+1} \geq a\right)$. We assume that, for some positive constants $\alpha_{x(. .)}$ and $\kappa_{x(. .,)}$, $\left|\phi\left(s, x\left(s, \omega_{j}\right), \omega\right)\right| \leq \alpha_{x(. . .)}+\kappa_{x(. . .)}\left|x\left(s, \omega_{j}\right)\right|$ for all $s, \omega_{j}$ and that $s \rightarrow \phi\left(s, x\left(s, \omega_{j}\right), \omega_{j}\right)$ for $s \in\left(\tau_{k}, \tau_{k+1}\right), k \geq j$ is Lipschitz continuous with rank $\hat{\kappa}_{x(. .)}\left(1+\kappa_{g}\right)^{k}$, for some constant $\hat{\kappa}_{x(., .)}$. Define $\left.\eta\left(r ; t, \omega_{j}\right):=E\left[\phi\left(r, x\left(r-, \omega_{j}\right), \omega_{j}\right) \mid t, \omega_{j}\right)\right]$, $r>t \geq a,\left(\eta\left(r, a, \omega_{j}\right)=\eta\left(r, a, \omega^{j}\right)\right.$. The symbol $\left.\mid t, \omega_{j}\right]$ means that the expectation is calculated by conditioning on all $\left(\tau_{i}, V_{i}\right)$ in $\omega_{j}$ for which $\tau_{i}<t$, (denote the largest such $i$ by $\left.i\left(\omega_{j}\right)\right)$, as well as on all $\left(\tau_{i}, V_{i}\right)$ in the fixed $\omega^{j}$. Then

$$
\partial^{+} \eta\left(t ; a, \omega^{j}\right) / \partial t=E\left[\gamma\left(t, \omega_{j}\right) \mid a, \omega^{j}\right],
$$

where $\left(\partial^{+} / \partial\right)$ means a right derivative and where $\gamma\left(t, \omega_{j}\right)=$

$$
\begin{aligned}
& \phi_{t}\left(t, x\left(t+, \omega_{j}\right), \omega\right)+\phi_{x}\left(t, x\left(t+, \omega_{j}\right), \omega\right) f\left(t, x\left(t+, \omega_{j}\right), u\left(t+, \omega_{j}\right), \omega\right)+ \\
& \left.\lambda\left(t, \omega_{j}\right) E\left[\phi\left(t, x\left(t+, \omega_{j}\right)+g\left(t, x\left(t+, \omega_{j}\right), V, w\left(t+, V, \omega_{j}\right), \omega\right), \omega_{i\left(\omega_{j}\right)}, t, V\right) \mid t, \omega_{j}\right)\right]- \\
& \lambda\left(t, \omega_{j}\right) \phi\left(t, x\left(t+, \omega_{j}\right), \omega\right) .
\end{aligned}
$$

the expectation being calculated by means of $\pi\left(v ; t+, \omega_{j}\right)$.

Proof of Lemma 1. First, we want to prove the following differential formula, (formula for the right derivative of $r \rightarrow \eta\left(r ; t, \omega_{j}\right)$ at $r=t$ ).

$$
\left[\partial^{+} \eta\left(r ; t, \omega_{j}\right) / \partial r\right]_{r=t}=\gamma\left(t, \omega_{j}\right)
$$

The proof of [88] is as follows. (In the proofs of [88] and Lemma 1 certain details have been relegated to points [A],[B] in Appendix). Write $X(t):=$ 
$x\left(t+, \omega_{j}\right)$. Assume that exactly $k$ jumps have occurred in $[0, t)$. For $\Delta t$ small, only a second order error is made by assuming that there is at most one jump point $\tau_{k+1}$ in $(t, t+\Delta t)$, with a probability approximately equal to $\lambda\left(t+, \omega_{j}\right) \Delta t$ for a jump,(see $[\mathrm{A}]$ in Appendix). Similarly, the approximation $w\left(t+, v, \omega_{j},\right)$ to the "true value" $w\left(\tau_{k+1}, v, \omega_{j}\right)$, (right limit assumption is used). By the continuity assumptions postulated, a first order error is made by assuming that $\phi\left(t+\Delta t, X(t+\Delta t), \omega_{k}, t, V_{k+1}\right)$ and $E\left[\phi\left(t+\Delta t, X(t+\Delta t), \omega_{k}, t, V_{k+1}\right) \mid \mathrm{a}\right.$ jump in $(t, t+\Delta t), X(t)]$ have roughly the same values irrespectively of where the jump $\tau_{k+1}$ occurs in $(t, t+\Delta t)$, we use the values these expressions have at $t+$. The expectation is calculated by means of $\left.\pi\left(. ; t+, \omega_{j}\right)\right)$, an approximation of the true distribution $\pi\left(. ; \tau_{k+1}, \omega_{j}\right)$ needed to calculate the expectation given that the jump occurs at $\tau_{k+1} \in(t, t+\Delta t)$. Using all these approximations, $E\left[\phi(t+\Delta t, X(t+\Delta t), \omega) \mid t, \omega_{j}\right]=$ $\phi(t+\Delta t, X(t+\Delta t), \omega) \operatorname{Pr}\left[\right.$ no jump in $\left.(t, t+\Delta t) \mid t, \omega_{j}\right]+$

$E\left[\phi(t+\Delta t, X(t+\Delta t), \omega) \mid\right.$ a jump in $\left.(t, t+\Delta t), t, \omega_{j}\right] \operatorname{Pr}[$ a jump in $(t, t+$ $\left.\Delta t) \mid t, \omega_{j}\right] \approx$

$$
\begin{aligned}
& \left(1-\lambda\left(t+, \omega_{j}\right) \Delta t\right)\left\{\phi\left(t, x\left(t+, \omega_{j}\right), \omega_{j}\right)+\phi_{t}\left(t, x\left(t+, \omega_{j}\right), \omega\right) \Delta t+\right. \\
& \left.\phi_{x}\left(t, x\left(t+, \omega_{j}\right), \omega\right) f\left(t, x\left(t+, \omega_{j}\right), u\left(t+, \omega_{j}\right), \omega\right) \Delta t\right\}+ \\
& \lambda\left(t+, \omega_{j}\right) \Delta t E\left[\phi\left(t, x\left(t+, \omega_{j}\right)+g\left(t, x\left(t+, \omega_{j}\right), V, w\left(t, V, \omega_{j}\right), \omega_{j}\right), \omega_{k}, t, V\right) \mid t, \omega_{j}\right] .
\end{aligned}
$$

Subtracting $\phi(t, X(t+), \omega)$ and dividing by $\Delta t$ and letting $\Delta t \rightarrow 0$, we get [88].

Next, let us find $\left(\partial^{+} / \partial r\right) \eta\left(r ; a, \omega^{j}\right)$ at $r=t>a$, i.e. prove Lemma 1 . Now, $\left[\left(\partial^{+} / \partial r\right) \eta\left(r ; a, \omega_{j}\right)\right]_{r=t}=$

$$
\begin{aligned}
& {\left[\left(\partial^{+} / \partial r\right) E\left[\phi\left(r, x\left(r-, \omega^{j}\right), \omega_{j}\right) \mid a, \omega_{j}\right]\right]_{r=t}=} \\
& {\left[\left(\partial^{+} / \partial r\right) E\left[E\left[\phi\left(r, x\left(r-, \omega^{j}\right), \omega_{j}\right) \mid t, \omega\right] \mid a, \omega^{j}\right]\right]_{r=t} .}
\end{aligned}
$$

When calculating the right derivative with respect to $r$ at $r=t$ of the righthand side, we can take the derivative inside the outer expectation sign. (An elaboration of this argument also shows that $\left[\left(\partial^{+} / \partial r\right) \eta\left(r ; a, \omega_{j}\right)\right]_{r=t}$ exists.) Then, by [88], Lemma 1 follows.

Let us next give an integral version of [87]. Assume given a solution $x\left(., \omega_{j}\right)$, starting in $\left(a, y, \omega^{j}\right)$, corresponding to $u\left(., \omega_{j}\right), w\left(., \omega_{j}\right)$. Then we claim that:

$$
\left.\left.E\left[\phi\left(b, x\left(b-, \omega_{j}\right), \omega\right) \mid a, \omega^{j}\right)\right]=\phi\left(a, x\left(a-, \omega^{j}\right), \omega\right)+E\left[\int_{a}^{b} \gamma\left(t, \omega_{j}\right) d t \mid a, \omega^{j}\right)\right] .[89]
$$

The property in [89] is sometimes also expressed by saying that for any $b$, $\kappa\left(b, \omega_{j}\right):=\phi\left(b, x\left(b-, \omega_{j}\right), \omega\right)-\int_{a}^{b} \gamma\left(t, \omega_{j}\right) d t$ is a martingale, (the martingale 
property: $\left.E\left[\kappa\left(b, \omega_{j}\right) \mid a, \omega^{j}\right)\right]=\kappa\left(a, \omega^{j}\right)$, for all $b>a$ holds. $)$

To prove [89], note first that it may be proved that $\eta\left(s ; a, \omega^{j}\right):=$ $\left.E\left[\phi\left(s, x\left(s-, \omega_{j}\right), \omega\right) \mid a, \omega^{j}\right)\right]$ is Lipschitz continuous,([B]). Now, a general result says that two continuous functions on $[a, b]$, with derivatives existing everywhere in $(a, b)$, are equal if their derivatives are equal, and their values at $a$ are equal. In this result, "derivatives" can be replaced by "right derivatives", if Lipschitz continuity prevails (and right derivatives exist). Replacing $b$ by $t \in[a, b]$ in [89] and taking right derivatives on both sides of [89] reveals that the two derivatives are equal, by [87]. Hence, [89] follows from the just mentioned theoretical result.

Note that a stopping time $\sigma(\omega)$ is a function such that $1_{[\sigma(\omega), b]}(t)$ is nonanticipating in $(t, \omega)$ and $\sigma(\omega)$ is right-continuous in each $\tau_{i}$, each component of each $V_{i}, \tau_{i}<t$.

Lemma 2 If in Lemma $1,(s, x) \rightarrow \phi\left(s, x\left(s, \omega_{j}\right), \omega\right)$ is $C^{1}$ in a neighborhood of all point $(s, x), s \in\left(\tau_{j}, \tau_{j+1}\right), x=x(s, \omega)$, except at the countable number of points $\left(\sigma_{k}^{j}(\omega), x\left(\sigma_{k}^{j}(\omega), \omega\right)\right), k=1,2, \ldots,\left(\sigma_{k}^{j}(\omega)\right.$ stopping times), then still [89] holds.

(The equality [89] even holds if $\sigma_{k}^{j}(\omega)$ is measurable in $\omega$.)

Proof of Lemma 2 Still $s \rightarrow E\left[\phi\left(s, x\left(s, \omega_{j}\right), \omega\right) \mid a, \omega^{j}\right]$ is Lipschitz continuous in $s \in[0, T]$. The derivative $(d / d s) E\left[\phi\left(s, x\left(s, \omega_{j}\right), \omega\right) \mid a, \omega^{j}\right]$ fails to exist on a null set. By [89], we have $E\left[\phi\left(b, x\left(b-, \omega_{j}\right), \omega\right)-\phi\left(a, x\left(a-, \omega_{j}\right), \omega\right) \mid a, \omega^{j}\right]=$

$\int_{a}^{b}\left\{(d / d r) E\left[\phi(r, x(r, \omega), \omega) \mid a, \omega^{j}\right]\right\}_{r=s} d s=$

$\left.\left.\int_{a}^{b}\left[d^{+} / d r\right)\left\{E[E[\phi(r, x(r, \omega), \omega)] \mid s, \omega] \mid a, \omega^{j}\right]\right\}\right]_{r=s} d s=$

$\int_{a}^{b} E\left[\left(d^{+} / d r\right) E[\phi(r, x(r, \omega), \omega) \mid s, \omega]_{r=s} \mid a, \omega^{j}\right] d s=E\left[\int_{a}^{b} \gamma\left(s, \omega_{j}\right) d s \mid a, \omega^{j}\right]$. The next to last equality follows from Lebesgue's dominated convergence theorem applied to $\left(E\left[\phi\left(s+h_{n}, x\left(s+h_{n}, \omega\right), \omega\right) \mid s, \omega\right]-E[\phi(s, x(s, \omega), \omega) \mid s, \omega]\right) / h_{n}, h_{n} \downarrow$ 0 , Lipschitz continuity, and the fact that a right derivative exists at all $s \neq \sigma_{k}^{j}(\omega)$.

Proof of Theorem 11 Let $x(t, \omega), u(t, \omega), w(t, v, \omega)$ be a $\left(0, x^{0}, \omega^{0}\right)$-admissible triple and let $\epsilon>0$ be arbitrary. Choose a $T^{\prime}$ close to $T$, such that $E J\left(T^{\prime}, x\left(T^{\prime}-, \omega\right), \omega\right) \geq E h_{0}(x(T-, \omega))-\epsilon$ and $E h_{0}\left(x^{*}(T-, \omega)\right) \geq$ $E J\left(T^{\prime}, x^{*}\left(T^{\prime}-, \omega\right), \omega\right)-\epsilon$, (use [85]). It is asserted that $J\left(0, x^{0}, \omega^{0}\right) \geq$ $E J\left(T^{\prime}, x\left(T^{\prime}-, \omega\right), \omega\right)$, with equality if $(x(.,),. u(.,),. w(.,))=$. 
$\left(x^{*}(.,),. u^{*}(.,),. w^{*}(.,).\right)$. To see this, by [82]-[84], $\gamma(t, \omega):=$ $\left[\left(\partial^{+} / \partial s\right) E[J(s, x(s, \omega), \omega) \mid t, \omega]\right]_{s=t} \leq 0$, with equality if $(x(.,),. u(.,),. w(.,))=$. $\left(x^{*}(.,),. u^{*}(.,),. w^{*}(.,).\right)$. Hence, by [89], the assertion is correct. Thus, $E J\left(T^{\prime}, x^{*}\left(T^{\prime}-, \omega\right), \omega\right)=J\left(0, x^{0}, \omega^{0}\right) \geq E J\left(T^{\prime}, x\left(T^{\prime}-, \omega\right), \omega\right)$, so $E h_{0}\left(x^{*}(T-, \omega)\right)+$ $\epsilon \geq J\left(0, x^{0}, \omega^{0}\right)=E h_{0}(x(T-, \omega))-\epsilon$. Since $\epsilon$ is arbitrary, $E h_{0}\left(x^{*}(T-, \omega)\right) \geq$ $E h_{0}(x(T-, \omega))$, so $\left(x^{*}(.,),. u^{*}(.,),. w^{*}(., .,).\right)$ is optimal.

Proof of Remark 24. Replacing the use of Lemma 1 by Lemma 2 in the above proof, yields automatically a proof of Remark 24 .

Proof of Theorem 12 For simplicity, it is assumed that $\lambda$ does not depend on $u$. Let $s, \omega^{j}$ be given, $\tau_{j}<s$. Let $(s, x) \in Q\left(\omega^{j}\right)$ and let $u\left(., \omega_{j}\right)$ and $w\left(., ., \omega_{j}\right)$ be arbitrary controls. Let $u\left(., \omega_{j}\right)$ be used on an interval $\left[s, \min \left\{\tau_{j+1}, t\right\}\right), t>s$, and if $\tau_{j+1}<t$, let $w\left(., ., \omega_{j}\right)$ govern the jump at $\tau_{j+1}$. By assumption, $Q\left(\omega^{j}\right)$ is open, so if $t-s$ is small enough, the corresponding solution $x\left(., \omega_{j}\right)$ (starting at $\left(s, x, \omega^{j}\right), \tau_{j+1} \geq s$ ) has the property that $x\left(t^{\prime}-, \omega_{j}\right)$ belongs to $Q\left(\omega^{j}\right)$ for all $t^{\prime} \in(s, t]$, as long as no jump occurs. If $\tau_{j+1}<t$, an optimal collection $u^{*}\left(., \omega_{j+1} ; t^{\prime}, y, \omega^{j+1}\right), w^{*}\left(., ., \omega_{j+1} ; t^{\prime}, y, \omega^{j+1}\right)$ is used from $\tau_{j+1}$ on, with $t^{\prime}=\tau_{j+1}, y=x^{u, w}\left(\tau_{j+1}+, \omega_{j}\right)$. By assumption such optimal controls exist for each $(s, y) \in Q\left(\omega^{j+1}\right)$. If $t \leq \tau_{j+1}, u^{*}\left(., \omega_{j}, t, y, \omega^{j}\right)$, $w^{*}\left(., ., \omega_{j}, t, y, \omega^{j}\right)$ is used on $[t, T]$, with $y=x^{u, w}\left(t-, \omega_{j}\right)$. Let $x^{u, w}\left(., \omega_{j}\right)$ correspond to the controls specified above, starting at $x$ at time $s$, (i.e. $\left.x^{u, w}\left(s-, \omega_{j}\right)=x\right)$. In particular, if $x=x^{*}(s-, \omega)$, then $\left(u^{*}\left(., \omega_{j}, t, y, \omega^{j}\right)\right.$, $\left.w^{*}\left(., ., \omega_{j}, t, y, \omega^{j}\right)\right)=\left(u^{*}(s, \omega), w^{*}(s, V, \omega)\right)$. The optimal reward obtained, when the state at $t$ is $x$ is $J^{*}\left(t, x, \omega_{j}\right)=\sup _{u^{\prime}, w^{\prime}} E\left[h_{0}\left(T, x^{u^{\prime}, w^{\prime}}\left(T-, \omega_{j}\right)\right) \mid t, \omega_{j}\right]$, all $x^{u^{\prime}, w^{\prime}}\left(., \omega_{j}\right)$ starting at $\left(t, x, \omega_{j}\right)$. (The reading of the symbol $\left.\mid t, \omega_{j}\right]$ is as above.) Now, for any $t>s$, for $x=x^{*}\left(s-, \omega^{j}\right)$

$$
\begin{aligned}
& J^{*}\left(s, x, \omega^{j}\right)=E\left[E\left[h_{0}\left(x^{u^{*}, w^{*}}\left(T-, \omega_{j}\right)\right) \mid t, x^{u, w}(t-, \omega), \omega_{j}\right] \mid s, x, \omega^{j}\right]= \\
& E\left[J^{*}\left(t, x^{u^{*}, w^{*}}\left(t-, \omega_{j}\right), \omega_{j}\right) \mid s, x, \omega^{j}\right]
\end{aligned}
$$

Furthermore, it can be shown that

$$
J^{*}\left(s, x, \omega^{j}\right) \geq E\left[J^{*}\left(t, x^{u, w}\left(t-, \omega_{j}\right)\right) \mid s, x, \omega^{j}\right]
$$

for $t>s$, with equality, if $s=t$. To show [91], note that $J^{*}\left(s, x, \omega^{j}\right) \geq$ $E\left[h_{0}\left(T, x^{u, w}(T-, \omega)\right) \mid s, x, \omega^{j}\right]=$ $E\left[E\left[h_{0}\left(T, x^{u, w}(T-, \omega)\right) \mid t, x^{u, w}(t-, \omega), \omega_{j}\right] \mid s, x, \omega^{j}\right]=E\left[J^{*}\left(t, x^{u, w}\left(t-, \omega_{j}\right)\right) \mid s, x, \omega^{j}\right]$, the last equality following from the fact that the pairs $\left(u^{*}\left(., \omega_{j}\right), w^{*}\left(., \omega_{j}\right)\right.$, $u^{*}\left(., \omega_{j}, t, y, \omega^{j}\right), w^{*}\left(., ., \omega_{j}, t, y, \omega^{j}\right)$ are optimal. The inequality [91] gives that the right derivative at $t=s$ of the right hand side is $\leq 0$, while [90] gives that the right derivative of the right-hand side equals 0 . The right derivative 
is the right-hand side of [82], with the sup's deleted. These results entails, in a shorthand notation, that for all $w(.,),. \lambda E[w(.,) \mid.] \leq \lambda E\left[w^{*}(.,) \mid.\right]$ at $\left(s, x^{*}\left(s-, \omega^{j}\right)\right)$, as well as [82] and [84]. The last inequality then gives [86], (omitting the proof that the expected value of the supremum can be approximated by $E[w(.,) \mid$.$] for some w(.,$.$) .)$

It can be proved that [82] holds even in the case where we drop the assumption about the existence of the $u^{*}\left(., \omega_{j} ; t, y\right), w^{*}\left(., \omega_{j} ; t, y\right)$, because [91] follows by another argument: It can be proved that

$$
\left.J^{*}\left(s, x, \omega^{j}\right)=\sup _{\hat{u}, \hat{w}} E\left[J^{*}\left(t, x^{\hat{u}, \hat{w}}\left(t-, \omega_{j}\right), \omega_{j}\right)\right) \mid s, x, \omega^{j}\right],
$$

where $\hat{u}:=\hat{u}\left(., \omega_{j}\right), \hat{w}:=\hat{w}\left(., V, \omega_{j}\right)$, and $x^{\hat{u}, \hat{w}}\left(., \omega_{j}\right)$ starts at $\left(s, x, \omega^{j}\right)$. For simplicity, consider only the case where the controls $w$ are absent. We shall only give a heuristic proof of $[92 \mathrm{~s}]$ and we write max instead of sup. Noting that any $\hat{u}$ can be written $\hat{u}=u^{\prime}$ for $r \in[s, t], \hat{u}=u^{\prime \prime}$, for $r \in(t, T]$, $J^{*}\left(s, x, \omega^{j}\right)=\max _{\hat{u}} E\left[h_{0}\left(x^{\hat{u}}(T-, \omega)\right) \mid s, x, \omega^{j}\right]=$

$$
\begin{aligned}
& \max _{u^{\prime}, u^{\prime \prime}} E\left[h_{0}\left(T, x^{u^{\prime}, u^{\prime \prime}}\left(T-\omega_{j}\right)\right) \mid s, x, \omega^{j}\right]= \\
& \max _{u^{\prime}}\left\{\max _{u^{\prime \prime}} E\left[h_{0}\left(T, x^{u^{\prime}, u^{\prime \prime}}\left(T-\omega_{j}\right)\right) \mid s, x, \omega^{j}\right]\right\}= \\
& \left.\max _{u^{\prime}}\left\{\max _{u^{\prime \prime}} E\left[E\left[h_{0}\left(T, x^{u^{\prime}, u^{\prime \prime}}\left(T-, \omega_{j}\right)\right) \mid t, x^{u^{\prime}}\left(t-, \omega_{j}\right), \omega_{j}\right] \mid s, x, \omega^{j}\right]\right]\right\}= \\
& \max _{u^{\prime}}\left\{E\left[\max _{u^{\prime \prime}} E\left[h_{0}\left(T, x^{u^{\prime}, u^{\prime \prime}}\left(T-\omega_{j}\right)\right) \mid t, x^{u^{\prime}}\left(t-, \omega_{j}\right), \omega_{j}\right] \mid s, x, \omega^{j}\right]\right\}= \\
& \max _{u^{\prime}} E\left[J^{*}\left(t, x^{u^{\prime}}\left(t-, \omega_{j}\right), \omega_{j}\right) \mid s, x, \omega^{j}\right] .
\end{aligned}
$$

The next to last equality involves an interchange of a maximization ( $\max _{u}$ ") and an expectation and needs the following argument: The outer expectation can be looked upon as a sum. The maximization of a sum equals the sum of the maximum of each term, provided each term contains a separate control parameter, not entering the other terms. This is the case here, for each realization of $\left.\mid t, \omega_{j}\right]$, a separate $u^{\prime \prime}$ can be chosen.

Proof of Theorem 13 In the next lemma, the following transversality condition is needed:

There exist multipliers $\mu_{j}^{*}(s, y), j=1, \ldots, m$, continuous in $(s, y)$ for $j=m^{\prime}+1, \ldots, m$,

such that $q(T ; s, y)=\Sigma \mu_{j}^{*}(s, y) h_{j x}(x(T ; s, y))+h_{0 x}(x(T ; s, y))$, $\mu_{j}^{*}(s, y) \geq 0, j>m^{\prime}, \mu_{j}^{*}(s, y)=0$ if $h_{j}(x(T ; s, y))>0, j>m^{\prime}$.

Lemma 3 Consider the control problem $\max _{u(.)}\left\{\int_{0}^{T} M^{*}(\rho, x) d \rho+h_{0}(x(T))\right\}$, subject to $\dot{x}=f(t, x, u), x(0)=x^{0}, h_{i}(x(T))=0, i=1, \ldots, m^{\prime}, h_{i}\left(x_{i}(T)\right) \geq$ $0, i=m^{\prime}+1, \ldots, m$, where $f$ is $C^{0} \times C^{2}$ as a function of $t$ and $(x, u)$, and where $h_{i}$ is $C^{2}$. Assume that $\hat{H}(t, x, q):=\max _{u \in U} q f(t, x, u)$ exists and 
is $C^{0} \times C^{2} \times C^{2}$ in an open set $Q^{*}$ in $\mathbb{R}^{2 n+1}$, and let $\hat{u}(t, x, q),(t, x, q) \in$ $Q^{*}$ be a $C^{0} \times C^{1} \times C^{1}$-function that yields this maximum. Assume that $Q$ is an open set in $(0, T) \times R^{n}$, and that for each $(s, y) \in Q$, continuous solutions $t \rightarrow(x(t ; s, y), q(t ; s, y))$ of $\dot{x}=f(t, x, \hat{u}(t, x, q)), x(s)=y$, $\dot{q}=-q f_{x}(t, x, \hat{u}(t, x, q))-M_{x}^{*}(t, x)$ exist on $[s, T]$, satisfying $x(s ; s, y)=y$, the transversality condition [93] and the just mentioned terminal conditions. Assume that $(s, y) \rightarrow(x(t ; s, y), q(t ; s, y))$ is $C^{1}$ in $(s, y) \in Q, t \in[s, T]$, that $(t, x(t ; s, y), q(t ; s, y)) \in Q^{*}$ for all $t \in[s, T]$, all $(s, y) \in Q$, and that $(t, x(t ; s, y)) \in Q$ for all $(s, y) \in Q$, all $t \in(s, T)$. Assume that $M^{*}(t, x)$ is $C^{0} \times C^{2}$ in $Q$, and that $\sup _{t \in[s, T)}\left|M^{*}(t, x(t ; s, y))\right|, \sup _{t \in[s, T)}\left|M_{x}^{*}(t, x(t ; s, y))\right|$, and $\sup _{t \in[s, T)}\left|M_{x x}^{*}(t, x(t ; s, y))\right|$ are locally bounded on $Q$. Define $z(t ; s, y)=$ $\int_{t}^{T} M^{*}(\rho, x(\rho ; s, y)) d \rho+h_{0}(x(T ; s, y))$. Then $S(s, y)=z(s ; s, y)$ is $C^{1}$ on $Q$, and satisfies the HJB- equation: $0=S_{s}(s, y)+\sup _{u}\left\{S_{y}(s, y) f(s, y, u)\right\}+$ $M^{*}(s, y)$ here. Moreover, $q(s ; s, y)=z_{y}(s ; s, y)=S_{y}(s, y)$ for $(s, y) \in Q$.

Proof of Lemma 3 Let $(\hat{s}, \hat{y})$ be a given point in $Q$ and define $\hat{q}=q(\hat{s}, \hat{s}, \hat{y})$. Define $A:=\{(t, x(t ; \hat{s}, \hat{y}), q(t ; \hat{s}, \hat{y})): t \in[\hat{s}, T]\}$. For some $\epsilon>0, B(A ; \epsilon):=$ $\{(t, x, q): \operatorname{dist}((t, x, q), A)<\epsilon\}$ is contained in $Q^{*}$. Since $q(\tilde{s} ; s, y)$ is close to $q(\tilde{s} ; \hat{s}, \hat{y})$ when $(s, y)$ is near $(\hat{s}, \hat{y}), \tilde{s}$ any number close to $\hat{s}, \tilde{s}>\hat{s}$, then, by standard theory of differential equations, a number $s^{\prime}<\hat{s}$ exists such that, for all $(s, y)$ near $(\hat{s}, \hat{y}), x(t ; s, y)$ and $q(t ; s, y)$ are defined on $\left[s^{\prime}, T\right]$, being $C^{1}$ in $(s, y)$ near $(\hat{s}, \hat{y})$, for any $t \in\left(s^{\prime}, T\right]$. Moreover, since $M_{x}^{*}(\rho ; x(\rho ; s, y))$ and $M_{x x}^{*}(\rho ; x(\rho ; s, y))$ are locally bounded in $(s, y)$, uniformly in $\rho$, and $q(\hat{s} ; s, y)$ is close to $q(\hat{s} ; \hat{s}, \hat{y})$ when $(s, y)$ is close to $(\hat{s}, \hat{y})$, then $x(t ; s, y), q(t ; s, y)$ are close to $x(t ; \hat{s}, \hat{y}), q(t ; \hat{s}, \hat{y})$, when $(s, y)$ is near $(\hat{s}, \hat{y})$, uniformly in $t \in\left[s^{\prime}, T\right)$, and, furthermore, $(s, y) \rightarrow x(t ; s, y)$ is Lipschitz continuous on a neighborhood of $(\hat{s}, \hat{y})$, uniformly in $t$, (the same property has $(s, y) \rightarrow, q(t ; s, y))$. This means that $z_{s}(t ; s, y)$ and $z_{y}(t, s, y)$ exist and are continuous near $(\hat{s}, \hat{y})$. Finally, the formula for $z(t ; s, y)$ evidently gives that $\dot{z}(t ; s, y)$ exist and is continuous. In fact, $z(s ; s, y)$ is $C^{1}$ in $Q$.

It will be shown that $q(T ; s, y) x_{y}(T ; s, y)=$

$h_{0 x}(x(T ; s, y)) x_{y}(T ; s, y), q(T ; s, y) x_{s}(T ; s, y)=h_{0 x}(x(T ; s, y)) x_{s}(T ; s, y)$. Тo see this, note that $\Sigma_{j} \mu_{j}^{*}(s, y) h_{j x}(x(T ; s, y))=\Sigma_{\mu_{j}^{*}(s, y) \neq 0} \mu_{j}^{*}(s, y) h_{j x}(x(T ; s, y))$. If $\mu_{j}^{*}(s, y)>0$, then, by continuity of $\left(s^{\prime}, y^{\prime}\right) \rightarrow \mu_{j}^{*}\left(s^{\prime}, y^{\prime}\right), j>m^{\prime}, \mu_{j}^{*}\left(s^{\prime}, y^{\prime}\right)>$ 0 for all $\left(s^{\prime}, y^{\prime}\right)$ in a neighborhood of $(s, y)$, hence $h_{j}\left(x\left(T ; s^{\prime}, y^{\prime}\right)\right)=0$ here, i.e. $(\partial / \partial y) h_{j}(x(T ; s, y))=0$ and $(\partial / \partial s) h_{j}(x(T ; s, y))=0$, thus $h_{j x}(x(T ; s, y)) x_{y}(T ; s, y)=0$ and $h_{j x}(x(T ; s, y)) x_{s}(T ; s, y)=0$. This evidently also holds for $j \leq m^{\prime}$, hence $\Sigma_{j} \mu_{j}^{*}(s, y) h_{j x}(x(T ; s, y)) x_{y}(T ; s, y)=0$ and $\Sigma_{j} \mu_{j}^{*}(s, y) h_{j x}(x(T ; s, y)) x_{s}(T ; s, y)=0$, so the two asserted equalities are 
satisfied.

Write $w(t ; s, y)=z_{y}(t ; s, y)-q(t ; s, y) x_{y}(t ; s, y)$ and $\hat{w}(t ; s, y)=z_{s}(t ; s, y)-$ $q(t ; s, y) x_{s}(t ; s, y)$. The two asserted equalities and $z(T ; s, y)=h_{0}(x(T ; s, y))$ imply

$$
\hat{w}(T ; s, y)=0, w(T ; s, y)=0 \text {. }
$$

Let $\hat{u} \in \operatorname{argmax}_{u} \hat{q} f(t, \hat{x}, u),(t, \hat{x}, \hat{q}) \in Q^{*}$. From the first order conditions for a local minimum at $(\hat{x}, \hat{q})$ of $\left(x^{\prime}, q^{\prime}\right) \rightarrow \hat{H}\left(t, x^{\prime}, q^{\prime}\right)-q^{\prime} f\left(t, x^{\prime}, \hat{u}\right)$, it follows that $\hat{H}_{\hat{x}}(t, \hat{x}, \hat{q})=\hat{q} \hat{f}_{x}(t, \hat{x}, \hat{u})$, and $\hat{H}_{\hat{q}}(t, \hat{x}, \hat{q})=f(t, \hat{x}, \hat{u})$. This means that $x(),. q($.$) , and z($.$) satisfy \dot{x}=F_{q}, \dot{q}=-F_{x}, \dot{z}^{*}=q F_{q}-F=-M^{*}$, where $F=\hat{H}(t, x, q)+M^{*}(t, x)$. These equations are the characteristic equations of the HJB-equation for $S$ above. By well-known results in the theory of nonlinear first-order partial differential equations, (see for example Seierstad (1998), p. 391),

$$
w_{t}(t ; s, y)=0, \hat{w}_{t}(t ; s, y)=0, \text { for all } t \in(s, T),
$$

which together with [94] gives $w(t ; s, y)=0, \hat{w}(t ; s, y)=0$ for all $t \in[s, T]$. Then, (using Seierstad (1998), p. 391 again), $S(s, y)=z(s ; s, y)$ is $C^{1}$ in $Q$, and, in $Q$, it satisfies the equation $0=S_{s}(s, y)+\hat{H}(s, y, q)+M^{*}(s, y)$, with $q=q(s ; s, y)=S_{y}(s, y)$. (For the $C^{1}$-property of $S(s, y)$, see a corresponding proof of the $C^{1} \times C^{2}$-property in the next proof.) I.e. the HJB-equation holds in $Q$.

Note If we want to apply Lemma 3 to a problem where an integral also appears in the criterion, i.e. to a problem where an additional state $x^{0}$ appears, with $\dot{x}^{0}=f_{0}(t, x, u), x^{0}(0)=0,\left(f_{0} C^{0} \times C^{2} \times C^{2}\right)$, and with criterion $x^{0}(T)+h_{0}(x(T))$, then even $(s, y) \rightarrow x^{0}(t ; s, y)$ need to be $C^{1}$ in $Q$, for $t \in[s, T]$. Now, given $(\hat{s}, \hat{y})$ in $Q$, the above existence of $B(A ; \epsilon)$ immediately entails the $C^{1}$-property of $x^{0}(t ; s, y)$.

Lemma 4 Assume in the situation of Lemma 3, that there exist a piecewise continuous control $u(t)=u\left(t ; 0, x^{0}\right)$, a solution $x(t)=x\left(t ; 0, x^{0}\right)$ of $\dot{x}=$ $f(t, x, u(t)), x(0)=x^{0}$, and a solution $q(t)=q\left(t ; 0, x^{0}\right)$ of $\dot{q}=-q f_{x}(t, x(t), u(t))-$ $M_{x}^{*}(t, x(t))$, satisfying the transversality and terminal conditions in Lemma 3 , the condition $(t, x(t)) \in Q$ for all $t \in(0, T)$, the maximum condition $q(t) f(t, x(t), u(t))=\hat{H}(t, x(t), q(t))$, and the "consistency condition" $\left(x\left(t^{\prime} ; t, x(t)\right), q\left(t^{\prime} ; t, x(t)\right)\right)=\left(x\left(t^{\prime}\right), q\left(t^{\prime}\right)\right)$, for all $t^{\prime} \geq t, t^{\prime}, t \in[0, T]$. Then,

$$
S_{s}(t, x(t))+S_{y}(t, x(t)) f(t, x(t), u(t))+M^{*}(t, x(t))=0
$$


and

$$
\lim _{t \uparrow T} S(t, x(t))=h_{0}(x(T)) .
$$

Proof of Lemma 4 By the consistency condition, $q(t)=q(t ; t, x(t))=$ $S_{y}(t, x(t))$, so by the maximum condition, [96] follows. Moreover, by consistency, since $z(t ; t, x(t))=\int_{t}^{T} M^{*}(\rho, x(\rho ; t, x(t))) d \rho+h_{0}(x(T ; t, x(t))=$ $\int_{t}^{T} M^{*}\left(\rho, x(\rho, T / 2, x(T / 2)) d \rho+h_{0}(x(T)), t>T / 2\right.$, by the boundedness property of $M^{*},[97]$ follows.

Lemma 5 Let $h_{j}, j=0, \ldots, m$, be $C^{2}$, let $f$ be $C^{0} \times C^{2}$ in $t$ and $(x, u)$, and let $\lambda(t)$ be piecewise continuous. Let $\hat{u}(t, x, p)$ be a $C^{1}$-function yielding maximum of $p f(t, x, u)$, for $u \in U$, for all $(t, x, p)$ in a given open set $Q^{*}$, and assume that $\hat{H}(t, x, p):=\max _{u \in U} p f(t, x, u)$ is $C^{0} \times C^{2} \times C^{2}$ here. Consider the equation $\dot{x}=f(t, x, u), x(0)=x^{0}$, with terminal conditions $h_{j}(x(T))=0, j=1, \ldots, m^{\prime}, h_{j}(x(T)) \geq 0, j=m^{\prime}+1, \ldots, m$. Assume that $Q$ is an open set in $(0, T) \times R^{n}$, and that for each $(s, y) \in Q \cup\left\{0, x^{0}\right\}$, there exist continuous solutions $t \rightarrow x(t ; s, y), p(t ; s, y)$ of $\dot{x}=f(t, x, \hat{u}(t, x, p))$ and $\dot{p}=-p f_{x}(t, x, \hat{u}(t, x, p))+\lambda(t) p-\lambda(t) M_{x}(t, x)$. Furthermore, assume that $(t, x(t ; s, y), p(t ; s, y))$ belongs to $Q^{*}$, for $t \in[s, T]$, for all $(s, y) \in Q$, that $(t, x(t ; s, y))$ belongs to $Q$ for $t \in(s, T)$, for all $(s, y) \in Q \cup\left\{0, x_{0}\right\}$, and that $(s, y) \rightarrow(x(t ; s, y), p(t ; s, y))$ is $C^{1}$ in $Q$ for $t$ in $[s, T]$. Assume that $M(t, x)$ is $C^{0} \times C^{2}$ in $Q$, and that $\sup _{t \in[s, T)}|M(t, x(t ; s, y))|, \sup _{t \in[s, T)}\left|M_{x}(t, x(t ; s, y))\right|$, and $\sup _{t \in[s, T)}\left|M_{x x}(t, x(t ; s, y))\right|$ are locally bounded on $Q$. Moreover, assume that, for all $(s, y) \in Q \cup\left\{\left(0, x^{0}\right)\right\}, x(t ; s, y), p(t ; s, y)$ satisfy the terminal conditions and the following transversality conditions: There exists multipliers $\mu_{j}(s, y), j=1, \ldots, m$, continuous in $(s, y) \in Q$ for $j=m^{\prime}+1, \ldots, m$, such that $p(T ; s, y)=\Sigma \mu_{j}(s, y) h_{j x}(x(T ; s, y))+h_{0 x}(x(T ; s, y)), \mu_{j}(s, y) \geq 0, j=$ $m^{\prime}+1, \ldots, m, \mu_{j}(s, y)=0$ if $h_{j}(x(T ; s, y))>0, j=m^{\prime}+1, \ldots, m$. Assume that $\left(x\left(t ; 0, x^{0}\right), p\left(t ; 0, x^{0}\right)\right)$ satisfies the consistency condition $\left(x\left(t^{\prime} ; t, x\left(t ;, 0, x^{0}\right)\right), p\left(t^{\prime} ; t, x\left(t ; 0, x^{0}\right)\right)\right)=\left(x\left(t^{\prime} ; 0, x^{0}\right), p\left(t^{\prime} ; 0, x^{0}\right)\right)$,

for all $t^{\prime} \geq t, t^{\prime}, t \in[0, T]$. Let $z(t ; s, y)$ be the solution of $\dot{z}=\lambda(t)\{z-$ $M(t, x(t ; s, y))\}, z(T)=h_{0}(x(T ; s, y))$. Then $J(s, y):=z(s ; s, y)$ is $C^{1}$ in $Q$ and satisfies the HJB-equation: $0=J_{s}(s, y)+\sup _{u}\left\{J_{y}(s, y) f(s, y, u)\right\}+$ $\lambda(s)\{M(s, y)-J(s, y)\}$. Moreover, $u\left(s ; 0, x^{0}\right):=\hat{u}\left(s ; x\left(s ; 0, x^{0}\right), p\left(s ; 0, x^{0}\right)\right)$ yields the supremum in this HJB-equation, when $y=x\left(s ; 0, x^{0}\right)$ and $\lim _{t \uparrow T} J\left(t, x\left(t ; 0, x^{0}\right)\right)=h_{0}\left(x\left(T ; 0, x^{0}\right)\right)$. Finally, $p(s ; s, y)=J_{y}(s, y),(s, y) \in$ $Q$. 


\section{Proof of Lemma 5}

Write $I(s, y)=J(s, y) \exp \left(\int_{T}^{s}-\lambda(\rho) d \rho\right)$. Then, we can show that $J$ satisfies the HJB-equation of Lemma 5 if and only if $I$ satisfies the HJB-equation of Lemma 4 . The fact that $J$ satisfies the HJB-equation is equivalent to

$$
\begin{aligned}
& 0=J_{s}(s, y) \exp \left(\int_{T}^{s}-\lambda(\rho) d \rho\right)+\max _{u}\left\{J_{y}(s, y) \exp \left(\int_{T}^{s}-\lambda(\rho) d \rho\right) f(s, y, u)\right\}+ \\
& \lambda(s) M(s, y) \exp \left(\int_{T}^{s}-\lambda(\rho) d \rho\right)-\lambda(s) J(s, y) \exp \left(\int_{T}^{s}-\lambda(\rho) d \rho\right) .
\end{aligned}
$$

I.e. $I(s, y)$ satisfies $0=I_{s}+\sup I_{y} f+M^{*}$, where $M^{*}(s, y)=\lambda(s) M(s, y) \exp \left(\int_{T}^{s}-\lambda(\rho) d \rho\right)$. Hence Lemma 5 follows from Lemma 4 , as $x(t ; s, y), q(t ; s, y)=$ $p(t ; s, y) \exp \left(\int_{T}^{t}-\lambda(\rho) d \rho\right)$ satisfy the conditions in Lemma 4 .

\section{Final step in the proof of Theorem 13}

Let us apply Lemma 5 for $[0, T]$ replaced by $\left[\tau_{j}, T\right], M(t, x)=E\left[\sup _{w} J(t, x+\right.$ $\left.\left.g\left(t, x, V_{j+1}, w, \omega^{j}\right), \omega^{j}, t, V_{j+1}\right) \mid t, \omega^{j}\right]$. Evidently, Lemma 5 implies that for each $\omega^{j}$, the HJB-equation in Theorem 11 holds in $Q^{0}\left(\omega^{j}\right)$. Moreover, $u\left(t, \omega^{j}\right)$, and $w\left(t, v, \omega^{j}\right)$, for $t>\tau_{j}$, yield the suprema in the HJB-equation for $y=$ $x\left(t-, \omega^{j}\right)$. Since, by assumption [85] holds, then all conditions in Theorem 11 are satisfied, hence $(u(t, \omega), w(t, v, \omega))$ is optimal.

\section{Proof of Theorem 3}

In the situation of Theorem 3 , for any given $j, J(s, y, j)=$ $h_{0}(x(T ; s, y))+\int_{s}^{T} e^{\lambda(s-\rho)}\left[f_{0}(\rho, x(\rho ; s, y, j), \hat{u}(\rho ; x(\rho ; s, y, j), p(\rho ; s, y, j)))+\right.$ $J(\rho, x(\rho ; s, y)+g(\rho, x(\rho ; s, y, j), j+1), j+1)] d \rho$.

We consider only the case where $f_{0}=0$, for the general case, however, take notice of Note to Lemma 3. By induction, assume that $J(s, y, j+1)$ is $C^{1} \times C^{2}$ on $Q^{0}(j)$ and that $J(s, y, j+1), J_{y}(s, y, j+1)$, and $J_{y y}(s, y, j+1)$ are bounded on bounded subsets of $Q^{0}(j)$. (This surely holds for $j=N$, since what follows works by putting $J(s, y, N+1)=0$.) Since $J(s, y, j+1)$ is bounded on bounded subsets, the local boundedness properties of $M(t, x, j)=J(t, x+$ $g(t, x, j+1), j+1), M_{x}(t, x, j)$, and $M_{x x}(t, x, j)$ are satisfied. In fact, Lemma 5 gives that $J(s, y):=J(s, y, j)$ is $C^{1}$ in $Q^{0}(j)$, and satisfies the HJBequation, and $J_{y}(s, y, j)=p(s ; s, y, j)$, (which even implies that $J_{y y}(s, y, j)=$ $p_{y}(s ; s, y, j)$ exists and is continuous.) This means that $I(s, y, j):=I(s, y)=$ $J(s, y) \exp (\lambda(j)(T-s))$ satisfies the HJB-equation of Lemma 3 , with $M^{*}(s, y):=$ $M^{*}(s, y, j)=\lambda(j+1) J(t, x+g(t, x, j+1), j+1) \exp (\lambda(j+1)(T-s))$, Since $\hat{u}(t, x, p, j), M^{*}(s, y, j)$ and $I_{y}(s, y, j)=p(s ; s, y, j) \exp (\lambda(j)(T-t))$ 
are bounded on bounded subsets of their domains of definition, $I_{s}(s, y, j)$ is also bounded on bounded subsets of $Q^{0}(j)$, by the HJB-equation. So even $I(s, y, j)$ is bounded on bounded subsets, and the same holds for $J(s, y, j)$, together with $J_{y}(s, y, j)$ and $J_{y y}(s, y, j)$. Since $u(t ; s, y, j)$ yields the maximum in the HJB-equation when $y$ in that equation is replaced by $x(t ; s, y, j)$ and [22] implies [85], then, by Theorem 11, Theorem 3 follows.

Proof of Theorem 6 The theorem follows directly from Theorem 13, once it is noted that [49] implies [85].

Lemma 6 In the situation of Lemma 4, weaken the differentiability assumptions on $\hat{H}(t, x, q), \hat{u}(t, x, q)$ and $(s, y) \rightarrow(x(t ; s, y), q(t ; s, y))$ as follows: Assume that there exist $C^{1}$-functions $\phi_{k}, k=1, \ldots, k^{*}$, such that, for any point $(t, x, q)$ in $Q^{*}, \phi_{k}(t, x, q)=0$ for at most one $k$ and such that $\hat{H}$ is $C^{0} \times C^{2} \times C^{2}$ in $Q^{*} \backslash Z, Z=\left\{(t, x, q): \phi_{i}(t, x, q)=0\right.$ for some $\left.i\right\}$. Assume that $\hat{u}(t, x, q)$ is $C^{0} \times C^{1} \times C^{1}$ in $Q^{*} \backslash Z$. Assume also that $\left.\hat{u}(t, x, q)\right|_{A},\left(\left.\right|_{A}\right.$ means restricted to $A$ ), has a $C^{0} \times C^{1} \times C^{1}$-extension to an open set containing $\operatorname{cl} A$, for any set $A$ of the form $\cap_{i} \Phi^{i}, \Phi^{i}=\left\{(t, x, q) \in Q^{*}: \phi_{i}(t, x, q)>0\right\}$, or $\Phi^{i}=\left\{(t, x, q) \in Q^{*}: \phi_{i}(t, x, q)<0\right\}$, (the direction of the inequality sign may depend on $i)$.

Let $Z^{\prime}:=\{(s, y) \in Q:(s, y, q(s ; s, y)) \in Z\}$. Assume that for any $(s, y) \in$ $Q \backslash Z^{\prime}, t \in[s, T], t \notin Q_{s, y}, Q_{s, y}:=\left\{t \in[s, T): \phi_{k}(t, x(t ; s, y), q(t ; s, y))=0\right.$ for some $k\},\left(s^{\prime}, y^{\prime}\right) \rightarrow\left(x\left(t, s^{\prime}, y^{\prime}\right), q\left(t ; s^{\prime}, y^{\prime}\right)\right)$ is $C^{1}$ in a neighborhood of $(s, y)$. Finally, for $(s, y) \in Q \cup\left\{\left(0, x^{0}\right)\right\}, t \in(s, T)$, for $u(t ; s, y):=\hat{u}(t, x(t ; s, y), q(t ; s, y))$ $\phi_{k}(t, x(t ; s, y), q(t ; s, y))=0 \Rightarrow \phi_{k t}(t, x(t ; s, y), q(t ; s, y))+$ $\phi_{k x}(t, x(t ; s, y), q(t ; s, y)) \dot{x}\left(t^{ \pm} ; s, y\right)+\phi_{k q}(t, x(t ; s, y), q(t ; s, y)) \dot{q}\left(t^{ \pm} ; s, y\right) \neq$ 0

(Either the expression is $>0$ for both the left and right limits, or the expression is $<0$ for both limits.) Then $S(s, y)$ is $C^{1}$ and satisfies the HJB-equation only for $(s, y) \in Q \backslash Z^{\prime}$, and [96] holds for all $\left(t, x\left(t ; 0, x^{0}\right)\right) \notin Z^{\prime}$, (i.e. all $t$ except a countable number).

Proof Write $v=(s, y)$ and let $\hat{v}:=(\hat{s}, \hat{y})$ belong to $Q \backslash Z^{\prime}$. We consider only the case where there is only one function $\phi$, and only one "crossing point" $t=\hat{t}$ in $(\hat{s}, T)$, where $\phi(\hat{t}, x(\hat{t} ; \hat{v}), q(\hat{t} ; \hat{v}))=0$, the general case being an easy extension. Let us prove that $w(t)=w(t ; \hat{s}, \hat{y})$ and $\hat{w}(t)=$ $\hat{w}(t, \hat{s}, \hat{y})$ have equal left and right limits at $t=\hat{t}$, then again it follows that $w(t) \equiv 0, \hat{w}(t) \equiv 0$, for $t \neq \hat{t}$, which will again finish the proof, since [94] holds. By the extension property of $\hat{u}(t, x, q)$, the solution $(x(t ; v), q(t ; v))$ on $[s, \hat{T}(v))$, for $v$ close to $\hat{v}$ has an extension $\left(x^{-}(t ; v), q^{-}(t ; v)\right)$ to a slightly 
larger interval, in fact to an interval $\left[t^{\prime \prime}, t^{\prime}\right], t^{\prime \prime}<\hat{s}, t^{\prime}>\hat{t}$, with $v \rightarrow$ $\left(x^{-}(t ; v), q^{-}(t ; v)\right)$ being continuously differentiable near $\hat{v}$ for all $t$ in $\left[t^{\prime \prime}, t^{\prime}\right]$, and similarly, the solution $(x(t ; v), q(t ; v))$ on $(\hat{T}(v), T)$ has an extension $\left(x^{+}(t ; v), q^{+}(t ; v)\right)$ to an interval $\left[t^{\prime \prime}, T\right], t^{\prime \prime}<\hat{t}$, with $v \rightarrow\left(x^{+}(t ; v), q^{+}(t ; v)\right)$ being continuously differentiable near $\hat{v}$, for all $t$ in $\left[t^{\prime \prime}, T\right]$. By the "nontangentiallity" condition $\left[97^{*}\right]$ in Lemma 6 , a $C^{1}$-function $\hat{T}(v), v$ near $\hat{v}$, exists such that $\hat{T}(\hat{v})=\hat{t}$, and $\phi\left(\hat{T}(v), x^{-}(\hat{T}(v) ; v), q^{-}(\hat{T}(v) ; v)\right)=0$. Now, for $t^{*}>\hat{t}, t^{*}$ close to $\hat{t}$, as $x^{+}\left(t^{*} ; v\right)=x^{-}(\hat{T}(v), v)+\int_{\hat{T}(v)}^{t^{*}} \dot{x}^{+}(s ; v)$, then, neglecting the small term (integral) arising from differentiating under the integral sign, $x_{v}^{+}\left(t^{*} ; v\right) \approx-\dot{x}^{+}(\hat{T}(v) ; v) \hat{T}^{\prime}(v)+\dot{x}^{-}(\hat{T}(v) ; v) \hat{T}^{\prime}(v)+x_{v}^{-}(\hat{T}(v) ; v)$. By the fact that $\hat{u}(\hat{t}-, x(\hat{t}-; \hat{v}), q(\hat{t}-; \hat{v}))$ and $\hat{u}(\hat{t}+, x(\hat{t}+; \hat{v}), q(\hat{t}+; \hat{v}))$ maximize the Hamiltonian $q f$ at $\hat{t}$, then $\left.q(\hat{t} ; \hat{v}) \dot{x}^{+}(\hat{t} ; \hat{v})\right)=q(\hat{t} ; \hat{v}) \dot{x}^{-}(\hat{t} ; \hat{v})$, or, $q(\hat{t} ; \hat{v}) x_{v}^{+}\left(t^{*} ; \hat{v}\right) \approx q(\hat{t} ; \hat{v}) x_{v}^{-}(\hat{t} ; \hat{v})$. Letting $t^{*} \downarrow \hat{t}, q(\hat{t} ; \hat{v}) x_{v}(\hat{t}+; \hat{v})=q(\hat{t} ; \hat{v}) x_{v}(\hat{t}-; \hat{v})$. It is easily shown that $z_{v}(t ; \hat{v})=\int_{t}^{T} M_{x}^{*} x_{v}(\rho ; \hat{v})+(d / d v) h_{0}(x(T ; \hat{v}))$ exists and is continuous in $t$, for a proof in a more general situation, see the proof of Lemma 7 below. Hence the equality of left and right limits at $\hat{t}$ of $w(t)$ and $\hat{w}(t)$ follows. Now, $w_{t}=0$ and $\hat{w}_{t}=0$, for $t \neq \hat{t}$ by the same proof as in Lemma 3. As [94] holds, $w(t ; s, y)=0$ and $\hat{w}(t ; s, y)=0$ for all $t \in[s, T]$, and the proof is finished.

Lemma 7 In the situation of Lemma 6 , define $Z^{2}:=\{(s, y) \in Q$ :

$\phi_{k}(T, x(T ; s, y), q(T ; s, y))=0$ for some $\left.k=1, \ldots, k^{*}\right\}, Z^{1}=\{(s, y) \in Q:$ $(s, y, q(s ; s, y)) \in Z\}$, and redefine the set $Z^{\prime}$ in Lemma 6 to be $Z^{1} \cup Z^{2}$, (assuming that the conditions in Lemma 6 related to $Z^{\prime}$ holds for this redefinition). Assume that $M^{*}$ is $C^{0} \times C^{2}$ only in $Q \backslash Z^{\prime}$, and that

$\sup _{t \in[s, T) \backslash Q_{s, y}}\left|M^{*}(t, x(t ; s, y))\right|, \sup _{t \in[s, T) \backslash Q_{s, y}}\left|M_{x}^{*}(t, x(t ; s, y))\right|$, and $\sup _{t \in[s, T) \backslash Q_{s, y}}\left|M_{x x}^{*}(t, x(t ; s, y))\right|$ are locally bounded on $Q \backslash Z^{\prime}$. It is assumed that for any $(s, y) \in Q \backslash Z^{\prime}$, a $C^{0} \times C^{2}$-extension of $M^{*}$ to an open set containing $\{(t, x(t ; s, y)): t \in[a, b]\}$ exists for any interval $[a, b] \subset(s, T)$ for which $(t, x(t, s, y)) \notin Z^{\prime}$ for $t \in(a, b)$. Then the conclusions in Lemma 6 still hold.

Proof Let us first show that it is still meaningful to talk about about solutions to the equations for $x$ and $q$ : We can, by insertion of $x(t ; s, y)$ and $q(t ; s, y)$ in the equation for $x$, test if this equation is satisfied v.e., $(\hat{u}(t, x(t ; s, y), q(t ; s, y))$ has at most a countable number of discontinuity points). Moreover, for $t \notin Q_{s, y},(t, x(t ; s, y), q(t ; s, y))=$ $\left(t, x(t ; s, y), q(t ; t, x(t ; s, y)) \notin Z\right.$, i.e, $(t, x(t, s, y)) \notin Z^{\prime}$, so $M^{*}$ is $C^{0} \times C^{2}$ near $(t, x(t, s, y))$ and for all such $t$ (i.e. all $t$ except a countable number), the equation for $q$ can be tested. To see that the proof of Lemma 6 goes through even 
in the present case, simply let $M^{*-}$ and $M^{*+}$ be $C^{0} \times C^{2}$ extensions of $M^{*}$ to open sets $G$ and $G^{\prime}$ containing $\{(t, x(t ; \hat{s}, \hat{y})): t \in[\hat{s}, \hat{t}]\}$ and $\{(t, x(t ; \hat{s}, \hat{y}))$ : $t \in[\hat{t}, \hat{T}]\}$, respectively, $\hat{T}$ any given number in $(\hat{t}, T)$. Now, use $M^{*}=M^{*-}$ and $M^{*}=M^{*+}$ when defining $q^{-}(t, v)$ and $q^{+}(t, v)$. Again, the local boundedness of $M_{x}^{*}(\rho ; x(\rho ; s, y))$ and $M_{x x}^{*}(\rho ; x(\rho ; s, y))$ gives, as in Lemma 3 , that $x(t ; s, y), q(t ; s, y)$ are close to $x(t ; \hat{s}, \hat{y}), q(t ; \hat{s}, \hat{y})$, when $(s, y)$ is near $(\hat{s}, \hat{y})$, uniformly in $t \in\left[s^{\prime}, T\right)$., Furthermore, $(s, y) \rightarrow x(t ; s, y)$ is Lipschitz continuous in some ball $B((\hat{s}, \hat{y}), \delta)$, uniformly in $t \notin(\hat{t}-\epsilon(\delta), \hat{t}+\epsilon(\delta))$, where $\epsilon(\delta)>$ $0, \lim _{\delta \rightarrow 0} \epsilon(\delta)=0$. Using the local boundedness of $M_{x}^{*}(\rho ; x(\rho ; s, y))$, evidently, $(s, y) \rightarrow z(t ; s, y):=\int_{t}^{T} M^{*} d \rho+h_{0}(X(T ; s, y))$ is $C^{1}$ for $(s, y) \in Q \backslash Z^{\prime}$, with $t \rightarrow z_{v}(t ; v)$ continuous and $(s, y) \rightarrow z(s ; s, y) C^{1}$ for $(s, y) \in Q \backslash Z^{\prime}$. Then the arguments in Lemma 6 goes through as before. (Note that in Lemma 6 , it is implicitly assumed that $\left(s^{\prime}, y^{\prime}\right) \rightarrow\left(x\left(T ; s^{\prime}, y^{\prime}\right), q\left(T ; s^{\prime}, y^{\prime}\right)\right)$ is $C^{1}$ near $(s, y)$ even if $\phi_{k}(T, x(T ; s, y), q(T ; s, y))=0$ for some $k$, however, when $(s, y) \notin Z^{1} \cup Z^{2}$, none of these equalities hold.)

Lemma 8 For $k=1, \ldots, k^{*}$, define $\Phi_{k}:=\left\{(s, y) \in Q: \phi_{k}(s, y, q(s ; s, y))=0\right\}$, and define $\Phi_{k^{*}+j}:=\left\{(s, y) \in Q: \phi_{j}(T, x(T ; s, y), q(T ; s, y))=0\right\}$. Assume in the situation of Lemma 7, that, for any admissible solution $x(),$.$Q contains$ all points $(s, x(s)), s \in(0, T)$ and that for any $k=1, \ldots, 2 k^{*}$, for any $s$ such that $(s, x(s)) \in \Phi_{k}$ a number $\epsilon>0$ exists, such that for $s^{\prime}$ in $(s, s+\epsilon)$, $\left(s^{\prime}, x\left(s^{\prime}\right)\right) \notin \Phi_{k}$. Then except for a countable number of points $s,(s, x(s))$ belongs to $Q \backslash Z^{\prime}$.

Proof For any $k$, there is a finite set $I_{m}$ of points $s$ such that $(s, x(s)) \in \Phi_{k}$ and $\left(s^{\prime}, x\left(s^{\prime}\right)\right) \notin \Phi_{k}$ for $s^{\prime} \in(s, s+1 / m)$. In fact, the number of points in $I_{m}$ must be $\leq T /(1 / m)=m T$. Evidently, $\left\{s:(s, x(s)) \in \Phi_{k}\right\} \subset \cup_{m} I_{m}$.

Remark 26. In the case of Lemma $8, u\left(t ; 0, x^{0}\right)$ will be optimal in the problem of Lemma 3, provided the following condition holds: For each $(T, \tilde{y}) \in \operatorname{cl} Q$ a ball $N:=B((T, \tilde{y}), a)$ exists such that

$\left.\sup _{(s, y) \in Q \cap N, t \in[s, T]} \mid u(t ; s, y)\right) \mid<\infty$. This condition can even be replaced by the weaker condition that $\lim _{t \rightarrow} J(t, x(t))=h_{0}(x(T))$ for any admissible $x($.$) . (To see that the former condition implies the latter one, note that$ for $\tilde{y}=x(T), \sup _{(s, y) \in Q \cap N, t \in[s, T]}|f(t, y, u(t ; s, y))|=\Xi<\infty$, so that for $(s, y) \in B((T, \tilde{y}),(\min \{a, a / \Xi\}) / 4),(\rho, x(\rho ; s, y)$ belongs to $B((T, \tilde{y}), a)$ for $\rho \geq s$, as $|x(\rho ; s, y)-y| \leq \Xi(t-s) \leq a / 4,|y-\tilde{y}|<a / 4$. By the boundedness property of $M^{*}, J(t, x(t)) \rightarrow h_{0}(x(T))$.) 


\section{Remark 27 (Weakened differentiability assumptions on the char- acteristic solutions.)}

The differentiability conditions on $x\left(t ; s, y, \omega^{j}\right), p\left(t ; s, y, \omega^{j}\right), \hat{u}\left(t, x, p, \omega^{j}\right)$, $\hat{H}\left(t, x, p, \omega^{j}\right)$ and $M\left(t, s, \omega^{j}\right)$ in Theorem 13 can be weakened as follows. Let $\phi_{k}\left(t, x, p, \omega^{j}\right), k=1, \ldots, k_{j}^{*}$, be $C^{1}$-functions in $(t, x, p)$, nonanticipating and piecewise and right-continuous in each $\tau_{i}, \tau_{i}<t$, each component of each $V_{i}$, and assume that for any given $\omega^{j}$, for any $(s, y) \in Q^{0}\left(\omega^{j}\right)$, if

$$
\begin{aligned}
& t \in(s, T) \text { and } \phi_{k}\left(t, x\left(t ; s, y, \omega^{j}\right), p\left(t ; s, y, \omega^{j}\right), \omega^{j}\right)=0, \text { then } \\
& \phi_{k t}+\phi_{k x}(\partial / \partial t) x\left(t^{ \pm} ; s, y, \omega^{j}\right)+\phi_{k p}(\partial / \partial t) p\left(t^{ \pm} ; s, y, \omega^{j}\right) \neq 0,
\end{aligned}
$$

[98],

the partial derivatives $\phi_{k t}, \phi_{k x}$ and $\phi_{k p}$ being evaluated at $\left(t, x\left(t ; s, y, \omega^{j}\right)\right.$, $\left.p\left(t ; s, y, \omega^{j}\right), \omega^{j}\right)$. (The expression on the left-hand side of the "nonequality" in [98] is assumed to be either $>0$ for both the right and left limit, or $<0$ for both limits.) Assume that $\hat{H}\left(t, x, p, \omega^{j}\right)$ is $C^{0} \times C^{2} \times C^{2}$ in $\left\{(t, x, p) \in Q^{*}\left(\omega^{j}\right): \phi_{k}\left(t, x, p, \omega^{j}\right) \neq 0\right.$ for all $\left.k\right\}$ and that $\hat{u}(t, x, p)$ is $C^{0} \times C^{1} \times C^{1}$ here. Assume also that for each $\omega^{j},\left.(t, x, p) \rightarrow \hat{u}\left(t, x, p, \omega^{j}\right)\right|_{A}$ has a $C^{0} \times C^{1} \times C^{1}$-extension to an open set containing $\operatorname{cl} A$, for any set $A$ of the form $\cap_{i} \Phi^{i}, \Phi^{i}=\left\{(t, x, p) \in Q^{*}\left(\omega^{j}\right): \phi_{i}\left(t, x, p, \omega^{j}\right)>0\right\}$, or $\Phi^{i}=\{(t, x, p) \in$ $\left.Q^{*}\left(\omega^{j}\right): \phi_{i}\left(t, x, p, \omega^{j}\right)<0\right\}$, (the direction of the inequality sign may depend on $i$ ). Let $Q^{1}\left(\omega^{j}\right):=\left\{(s, y) \in Q^{0}\left(\omega^{j}\right): \phi_{k}\left(s, y, p\left(s ; s, y, \omega^{j}\right), \omega^{j}\right)=0\right.$ for some $k\}, Q_{s, y, \omega^{j}}:=\left\{t \in[s, T): \phi_{k}\left(t, x\left(t ; s, y, \omega^{j}\right), p\left(t ; s, y, \omega^{j}\right), \omega^{j}\right)=0\right.$ for some $k\}$, and $Q^{2}\left(\omega^{j}\right):=\left\{(s, y) \in Q^{0}\left(\omega^{j}\right): \phi_{k}\left(T, x\left(T, s, y, \omega^{j}\right), p\left(T ; s, y, \omega^{j}\right)\right)=0\right.$ for some $\left.k=1, \ldots, k_{j}^{*}\right\}$ and assume that for any $(s, y) \in Q^{0}\left(\omega^{j}\right) \backslash\left(Q^{1}\left(\omega^{j}\right) \cup\right.$ $\left.Q^{2}\left(\omega^{j}\right)\right)$, for any $t \notin Q_{s, y, \omega^{j}},\left(s^{\prime}, y^{\prime}\right) \rightarrow\left(x\left(t ; s^{\prime}, y^{\prime}, \omega^{j}\right), p\left(t ; s^{\prime}, y^{\prime}, \omega^{j}\right)\right)$ is $C^{1}$ for all $\left(s^{\prime}, y^{\prime}\right)$ in a neighborhood $N_{t, s, y, \omega^{j}}$ of $(s, y)$. Assume that for any $(t, x, p) \in$ $\left.Q^{*}\left(\omega^{j}\right), \phi_{k}\left(t, s, p, \omega^{j}\right)\right)=0$ for at most one $k$. Assume that $M\left(s, y, \omega^{j}\right):=$ $E\left[\sup _{w} J\left(s, y+g\left(s, y, V, w, \omega^{j}\right), \omega^{j}, s, V\right) \mid s, \omega^{j}\right]$ is $C^{0} \times C^{2}$ in $Q^{0}\left(\omega^{j}\right) \backslash\left(Q^{1}\left(\omega^{j}\right) \cup\right.$ $\left.Q^{2}\left(\omega^{j}\right)\right)$. Assume that $\sup _{t \in[s, T) \backslash Q_{s, y, \omega^{j}}}|M(t, x(t ; s, y))|$,

$\sup _{t \in[s, T) \backslash Q_{s, y, \omega^{j}}}\left|M_{x}\left(t, x(t ; s, y), \omega^{j}\right)\right|$ and $\sup _{t \in[s, T) \backslash Q_{s, y, \omega^{j}}}\left|M_{x x}\left(t, x(t ; s, y), \omega^{j}\right)\right|$ are locally bounded on $Q^{0}\left(\omega^{j}\right) \backslash\left(Q^{1}\left(\omega^{j}\right) \cup Q^{2}\left(\omega^{j}\right)\right)$, and that for any $(s, y) \in$ $Q^{0}\left(\omega^{j}\right) \backslash\left(Q^{1}\left(\omega^{j}\right) \cup Q^{2}\left(\omega^{j}\right)\right), M\left(s^{\prime}, y^{\prime}, \omega^{j}\right)$ has a $C^{0} \times C^{2}$-extension to an open set around the set $\left\{\left(s^{\prime}, y^{\prime}\right)=\left(s^{\prime}, x\left(s^{\prime} ; s, y, \omega^{j}\right)\right): s^{\prime} \in[a, b]\right\}$, for any interval $[a, b] \subset(s, T)$ for which $(a, b) \cap Q_{s, y, \omega^{j}}=\emptyset$. Finally, assume that for any admissible solution $x(., \omega)$, for any $s>\tau_{j}$ such that $\left(s, x\left(s, \omega^{j}\right)\right)$ belongs to $Q^{1}\left(\omega^{j}\right) \cup Q^{2}\left(\omega^{j}\right)$, then, for some $\epsilon>0,\left(s^{\prime}, x\left(s^{\prime}, \omega^{j}\right)\right) \notin Q^{1}\left(\omega^{j}\right) \cup Q^{2}\left(\omega^{j}\right)$ for $s^{\prime} \in(s, s+\epsilon)$.

Proof of Remark 27 Similar to what was obtained in the finishing part of the proof of Theorem 13, the HJB-equation holds for all $(s, y) \in Q^{0}\left(\omega^{j}\right) \backslash$ 
$\left(Q^{1}\left(\omega^{j}\right) \cup Q^{2}\left(\omega^{j}\right)\right)$, by Lemma 7. By the last property in Remark 27 , for any admissible solution $x(., \omega), J\left(t, x, \omega^{j}\right)$ is continuously differentiable near any point $(s, x(s, \omega)), s \in\left(\tau_{j}, \tau_{j+1}\right)$, except for a countable number of $s$ 's. These points depend as in Remark 24 on $\omega^{j}$, or perhaps only "measurable", in which case the conclusions in Remark 24 still hold, (note that $Z^{\prime}=Q^{1}\left(\omega^{j}\right) \cup Q^{2}\left(\omega^{j}\right)$ is relatively open in $\left.\left[\tau_{j}, T\right) \times \mathbb{R}^{n}\right)$ ).

Proof of Remark 19. In case of Remark 19, $M\left(s, y, \omega^{j}\right)=z(s ; s, y+$ $g(s, y, j+1), j+1)$. Moreover, $\left(s^{\prime}, y^{\prime}\right) \rightarrow\left(x\left(T ; s^{\prime}, y^{\prime}, j\right), p\left(T ; s^{\prime}, y^{\prime}, j\right)\right)$ is assumed to be $C^{1}$ for $\left(s^{\prime}, y^{\prime}\right)$ near any $(s, y)$ in $\mathrm{Q}^{0}(j) \backslash Q^{1}(j)$, even if $(s, y)$ belongs to $\mathrm{Q}^{2}\left(\omega^{j}\right)=Q^{2}(j)$. From this comment, evidently Remark 19 follows.

Lemma 9 In case of Lemmas 3 and 7, in the free end case, we can drop the assumption of the continuous differentiability of $(s, y) \rightarrow(x(t ; s, y) ; q(t ; s, y))$, since it automatically holds.

Proof (Sketch). We consider only the case of Lemma 3, for $M^{*} \equiv 0$, a similar proof holds in case of Lemma 7 . Let $(\hat{s}, \hat{y})$ belong to $Q$, and let $(\tilde{x}(t ; s, y, p), \tilde{q}(t ; s, y, p))$ be the solution of $\dot{x}=f(t, x, \hat{u}(t, x, q))$, $\dot{q}-q f_{x}(t, x, \hat{u}(t, x, q)), \tilde{x}(s ; s, y, p)=y$, $\tilde{q}(s ; s, y, p)=p$. A solution exist at least for $(s, y, p)$ close to $(\hat{s}, \hat{x}, q(\hat{s} ; \hat{s}, \hat{x}))$. Then $(x(t ; s, y), q(t ; s, y))$ equals $\left(\tilde{x}\left(t ; s, y, p_{1}(y)\right), \tilde{q}\left(t ; s, y, p_{1}(y)\right)\right)$, where $p_{1}(s, y)$ is a solution of

$$
\tilde{q}(T ; s, y, p)-h_{0 x}(\tilde{x}(T ; s, y, p))=0 .
$$

At least this is so for $\hat{s}$ close to $T$, as we shall see. For $\hat{s}$ slightly smaller than $T, \tilde{q}(T ; \hat{s}, y, p)=p+\int_{s^{T}-q f_{x} d t}, \tilde{x}(T ; s, y, p)=y+\int_{s}^{T} f d t$. So for $\hat{s}$ close to $T,(\partial / \partial p)\left[\tilde{q}(T ; \hat{s}, y, p)-h_{0 x}(\tilde{x}(T ; \hat{s}, y, p))\right] \approx I$, using the two preceding equalities and disregarding the small terms arising from the integrals obtained by differentiating under the integral sign with respect to $p$. Hence, a unique $p_{1}(s, y)$ exists for $(s, y)$ close to $(\hat{s}, \hat{y})$, with $p_{1}(\hat{s}, \hat{y})=q(T ; \hat{s}, \hat{y})$, which is $C^{1}$, by the implicit function theorem. Fix a $\hat{s}=T_{1}$, for which this holds. Then consider the equation $\tilde{q}\left(T_{1} ; s, y, p\right)-p_{1}\left(\tilde{x}\left(T_{1} ; s, y, p\right)\right)=0$. Then, by exactly the same arguments, for $\hat{s}$ slightly smaller than $T_{1}$, a unique solution $p=p_{2}(s, y)$ of this equation exists for $(s, y)$ close to $(\hat{s}, \hat{y})$, with $p_{2}(\hat{s}, \hat{y})=q\left(T_{1} ; \hat{s}, \hat{y}\right)$, which is $C^{1}$, by the implicit function theorem. Now, using $(*)$ for $p=p_{1}(s, y), s=T_{1}, y=\tilde{x}\left(T_{1} ; s, y, p_{2}(s, y)\right)$ gives $0=$ $\tilde{q}\left(T ; T_{1}, \tilde{x}\left(T_{1} ; s, y, p_{2}(s, y)\right), p_{1}\left(T_{1}, \tilde{x}\left(T_{1} ; s, y, p_{2}(s, y)\right)\right)\right)-$ $h_{0 x}\left(\tilde{x}\left(T ; T_{1}, \tilde{x}\left(T_{1} ; s, y, p_{2}(s, y)\right), p_{1}\left(T_{1}, \tilde{x}\left(T_{1} ; s, y, p_{2}(s, y)\right)\right)\right)\right)=$ $\left.\tilde{q}\left(T ; T_{1}, \tilde{x}\left(T_{1} ; s, y, p_{2}(s, y)\right), \tilde{q}\left(T_{1} ; s, y, p_{2}(s, y)\right)\right)\right)-$ 
$\left.h_{0 x}\left(\tilde{x}\left(T ; T_{1}, \tilde{x}\left(T_{1} ; s, y, p_{2}(s, y)\right), \tilde{q}\left(T_{1} ; s, y, p_{2}(s, y)\right)\right)\right)\right)=$

$\tilde{q}\left(T ; s, y, p_{2}(s, y)\right)-h_{0 x}\left(x\left(T ; s, y, p_{2}(s, y)\right)\right)$. Fix a $\hat{s}=T_{2}<T_{1}$ such that this holds.

Carrying out several such steps, say $k$ times, we can reach back to any given $\hat{s}=T_{k}$, with $\tilde{q}\left(T ; s, y, p_{k}(s, y)\right)-h_{0 x}\left(\tilde{x}\left(T ; s, y, p_{k}(s, y)\right)\right)=0$ holding for a unique function $p_{k}(s, y)$ being $C^{1}$ close to $(s, y)$ close to $(\hat{s}, \hat{y}) \in Q$, with $p_{k}\left(T_{k}, \hat{y}\right)=q\left(T_{k-1} ; \hat{s}, \hat{y}\right)$. (It is possible to keep $T_{i}-T_{i+1}, i=1, \ldots, k-1$, independent of $i$.)

Remark 28 In Theorem 3, for $j=0,(s, y) \rightarrow(x(t ; s, y, j), p(t ; s, y, j))$ need only be $C^{0} \times C^{1}$ and for $j=0$, the boundedness properties of $p$ and $p_{y}$ are not needed. If $h_{0}$ is $C^{N+2}, f(., ., ., j)$ is $C^{0} \times C^{j+2} \times C^{j+2}, g(., ., j)$ is $C^{0} \times C^{j+2}$, and $\hat{u}(t, x, p, j)$ is $C^{0} \times C^{j+1} \times C^{j+1}$, then, for any $j,(s, y) \rightarrow$ $(x(t ; s, y, j), q(t ; s, y, j))$ is $C^{0} \times C^{j+1} \times C^{j+1}$, this suffices for Theorem 3 to hold. No $C^{1} \times C^{2}$-assumption on $(s, y) \rightarrow(x(t ; s, y, j), p(t ; s, y, j))$ is then needed. (A proof is obtained from an obvious extension of Lemma 9 that yield $C^{j+1}$-continuity of $(s, y) \rightarrow x(t ; s, y, j), p(t ; s, y, j)$.)

\section{Free terminal time $T$}

Now, the time $T$ is subject to choice in a given set $\left[T_{1}, T_{2}\right]$. The problem is to choose a triple $u(., \omega), w(., ., \omega), T=T(\omega)$, such that the maximum in [78] is obtained. The stopping time $T(\omega)$ depends on the history of the system, i.e. $1_{[0, T(\omega)]}(t)$ is required to be nonanticipating. Moreover, $T$ is separately piecewise and right-continuous in each $\tau_{i}$, each component of each $V_{i}$. The class of triples $(u(.,),. w(., .,),. T()$.$) , with corresponding \left(0, x^{0}, \omega^{0}\right)$ admissible solutions $x(., \omega)$ is now the class in which an optimal triple is sought for. Now, "admissible" includes the satisfaction of the given terminal conditions: A.s.,

$$
\begin{gathered}
x_{i}(T(\omega), \omega)=\hat{x}_{i}, i=1, \ldots, n^{\prime} \\
x_{i}(T(\omega), \omega) \geq \hat{x}_{i}, i=n^{\prime}+1, \ldots, n^{\prime \prime},
\end{gathered}
$$

Functions $\lambda(t, x, u, \omega)$ and $\pi(t, x, \omega)$, satisfying the same assumptions as above, again describe the probability properties of the $\tau_{j}$ 's and the $V_{j}$ 's. Now, $h_{0}(t, x)$ is assumed to be Lipschitz continuous with rank $\kappa_{h_{0}}$ also in $t$. We also assume that $f, g$ and $h_{0}$ are $C^{2}$. We shall give two sets of sufficient conditions. Assume that $Q\left(\omega^{j}\right) \subset\left[\tau_{j}, T\right) \times \mathbb{R}^{n}, j=0,1, \ldots$ are given sets with the property that the $Q\left(\omega^{j}\right)^{\prime}$ 's contain all possible reachable points in the process when exactly $j$ jumps have occurred, in the sense that, for any 
$\left(0, x^{0}, \omega^{0}\right)$-admissible quadruple $(x(t, \omega), u(t, \omega), w(t, v, \omega), T(\omega))$, and for any $\omega^{j}$, any $\tau_{j+1},\left(t, x\left(t+; \omega^{j}\right)\right)$ belongs to $Q\left(\omega^{j}\right)$, if $t \in\left[\tau_{j}, T(\omega)\right), t \leq \tau_{t+1}$. Define $Q(\omega):=\left\{(t, x)\right.$ : for some $j, t \in\left[\tau_{j}, \tau_{j+1}\right)$ and $\left.(t, x) \in Q\left(\omega^{j}\right)\right\}$. Recall that for sets $A$ and $B$ in $\mathbb{R}^{n}, A \subset B, A$ is called relatively open in $B$ if there exists an open set $C$ in $\mathbb{R}^{n}$ such that $A=B \cap C$.

Theorem 14 Given sets $D\left(\omega^{j}\right) \subset Q\left(\omega^{j}\right), D\left(\omega^{j}\right)$ relatively open in $Q\left(\omega^{j}\right)$, and a function $J(t, x, \omega)$, nonanticipating in $(t, \omega)$, defined on $D(\omega)=\{(t, x)$ : for some $j, t \in\left[\tau_{j}, \tau_{j+1}\right)$ and $\left.(t, x) \in D\left(\omega^{j}\right)\right\}$. Define

$$
J(t, x, \omega):=h_{0}(t, x) \text {, for }(t, x) \in Q(\omega) \backslash D(\omega) .
$$

Assume that $\left\{(t, x) \in Q\left(\omega^{j}\right): t<T_{1}\right\} \subset D\left(\omega^{j}\right)$, and that

$$
(t, x) \in D\left(\omega^{j}\right), t \in\left[T_{1}, T_{2}\right) \Rightarrow J\left(t, x, \omega^{j}\right)>h_{0}(t, x) .
$$

Assume also that $(s, y) \rightarrow J(s, y, \omega)$ is $C^{1}$ at any point $(s, y) \in D(\omega)$, thus $J(s, y, \omega)$ has a $C^{1}$-extension to a neighborhood around $(s, y)$. The extension, as well as its derivatives $J_{s}$ and $J_{x}$ are, separately, piecewise and rightcontinuous in each $\tau_{i}$ and in each component of each $V_{i}$. Assume that the HJB-equation [82] holds for $(s, y)$ in $D\left(\omega^{j}\right)$.

Assume that for all $\omega^{j}$, for all $(s, y) \in Q\left(\omega^{j}\right) \backslash D\left(\omega^{j}\right), s>T_{1}, u \in U$,

$$
\begin{aligned}
& h_{0 t}(s, y)+h_{0 x}(s, y) f\left(s, y, u, \omega^{j}\right)+ \\
& \lambda\left(s, y, u, \omega^{j}\right)\left\{E\left[\sup _{w \in W} J\left(s, y+g\left(s, y, V, w, \omega^{j}\right), \omega^{j}, s, V\right) \mid s, y, \omega^{j}\right]-\right. \\
& \left.h_{0}(s, y)\right\} \leq 0
\end{aligned}
$$

Furthermore, assume that for all $\omega^{j}$,

$\left.J\left(s, y, \omega^{j}\right)\right|_{D\left(\omega^{j}\right)}$ has a $C^{1}$ extension denoted $J^{* *}\left(s, y, \omega^{j}\right)$

to an open set containing $D\left(\omega^{j}\right) \cup\left(Q\left(\omega^{j}\right) \cap \partial D\left(\omega^{j}\right)\right)$,

where $\partial D\left(\omega^{j}\right)$ is the boundary of $D\left(\omega^{j}\right)$, and

$J_{t}^{* *}\left(s, y, \omega^{j}\right)-h_{0 t}(s, y)+\left(J_{x}^{* *}\left(s, y, \omega^{j}\right)-h_{0 x}(s, y)\right) f\left(s, y, u, \omega^{j}\right) \neq 0$

for all $(s, y) \in Q\left(\omega^{j}\right) \cap \partial D\left(\omega^{j}\right), u \in U, s \in\left(T_{1}, T_{2}\right)$.

Assume, furthermore that, for any admissible pair $x(t, \omega), T(\omega)$ for all $t \in$ $D_{x(. .)}(\omega):=\left\{t^{\prime}:\left(t^{\prime}, x\left(t^{\prime}, \omega\right)\right) \in D(\omega)\right\}$, the following inequality holds

$$
|J(t, x(t, \omega), \omega)| \leq \alpha_{x(. . .)}+\kappa_{x(. . .)}|x(t, \omega)|
$$

for some positive constants $\alpha_{x(. .)}, \kappa_{x(. .)}$. Assume also that $t \rightarrow J(t, x(t, \omega), \omega)$ 
is Lipschitz continuous with Lipschitz rank $\beta_{x(., .)}\left(1+\kappa_{g}\right)^{j}$, for some constant $\beta_{x(. . .)}$, on any interval $\left(s^{\prime}, s^{\prime \prime}\right) \subset D_{x(. . .)}(\omega) \cap\left(\tau_{j}, \tau_{j+1}\right)$. Assume, moreover, that there exists a pair of control functions $u^{*}(t, \omega), w^{*}(t, V, \omega)$, with corresponding admissible solution $x^{*}(t, \omega)$ defined on $\left[0, T^{*}(\omega)\right]$, which satisfy [83] and [84], i.e. which yield the suprema in the HJB-equation, for $s$ in $\left[0, T^{*}(\omega)\right]$. Define $T^{j}\left(\omega^{j}\right):=\sup \left\{t:\left(s, x^{*}\left(s, \omega^{j}\right)\right) \in D\left(\omega^{j}\right)\right.$ for all $\left.s \in\left[\tau_{j}, t\right)\right\} \geq \tau_{j},\left(=\tau_{j}\right.$ if the set is empty), and assume that $T^{*}(\omega)=\min \left\{T^{j}\left(\omega^{j}\right): T^{j}\left(\omega^{j}\right)<\tau_{j+1}\right\}$. Assume, finally, that, for any admissible pair $x(., \omega), T(\omega)$, for any $\omega$ and any $s \in\left[T_{1}, T(\omega)\right], s \in\left(\tau_{j}, \tau_{j+1}\right)$,

$$
\text { if }(s, x(s, \omega)) \in \partial D\left(\omega^{j}\right) \text {, then } \lim _{t \rightarrow s} J(t, x(t, \omega), \omega)=h_{0}(s, x(s, \omega)) .
$$

Then $\left(u^{*}(t, \omega), w^{*}(t, V, \omega), T^{*}(\omega)\right)$ is an optimal triple in the class of all triples $u(t, \omega), w(t, V, \omega), T(\omega)$.

Sometimes [102] does not hold. In particular this can happen in end constrained problems, in which case, however, the following condition may hold:

For any admissible solution $x(., \omega)$ and for any $\omega$, if $\left(t, x\left(t, \omega^{j}\right)\right) \in \partial D\left(\omega^{j}\right), t>$ $\tau_{j}$, then $\left(s^{\prime}, x\left(s^{\prime}, \omega^{j}\right)\right) \notin \partial D\left(\omega^{j}\right)$, for all $s^{\prime}$ such that $s^{\prime} \neq t, s^{\prime}>\tau_{j}, s^{\prime} \in$ $\left(s_{1}, s_{2}\right),\left(s_{1}, s_{2}\right)$ some interval containing $t$.

Theorem 15. Theorem 14 even holds when [102] is replaced by [105], and when it is assumed that for each admissible $x(., \omega), T(\omega), J\left(s, y, \omega^{j}\right)$ is merely $C^{1}$ in a neighborhood of $\left(s, x\left(s, \omega^{j}\right)\right), s \in\left(0, T\left(\omega^{j}\right)\right)$ except for a countable number of points $\sigma_{k}^{j}\left(\omega^{j}\right), k=1,2, \ldots, \sigma_{k}^{j}\left(\omega^{j}\right)$ piecewise an right-continuous in each $\tau_{j}$, each component of each $V_{j}$.

Proofs of Theorems 14 and 15 Let $(x(t, \omega), u(t, \omega), w(t, V, \omega), T(\omega))$ be an arbitrary admissible quadruple. By [102] or [105], for each $\omega$, there exist a countable number of points $s=\beta_{n}^{j}\left(\omega^{j}\right), n=1,2$, in $\left(\tau_{j}, T\left(\omega^{j}\right)\right.$ ), (with no accumulation points in this set), for which $\left(s, x\left(s, \omega^{j}\right)\right) \in \partial D\left(\omega^{j}\right)$, and for all $s$ in $\left(\tau_{j}, T(\omega)\right), s \notin\left\{\beta_{n}^{j}\left(\omega^{j}\right)\right\}_{n} \cup\left\{\sigma_{k}^{j}\left(\omega^{j}\right)\right\}_{k}, J$ is continuously differentiable near $\left(s, x\left(s, \omega^{j}\right)\right.$ ). (In case of [102], by contradiction, if there exists a sequence $s_{n} \rightarrow s,\left(s, x\left(s, \omega^{j}\right)\right) \in \partial D\left(\omega^{j}\right),\left(s_{n}, x\left(s_{n}, \omega^{j}\right)\right) \in \partial D\left(\omega^{j}\right)$, then by [104], $h_{0}\left(s_{n}, x\left(s_{n}, \omega^{j}\right)\right)=J\left(s_{n}, x\left(s_{n}, \omega^{j}\right), \omega^{j}\right)=J^{* *}\left(s_{n}, x\left(s_{n}, \omega^{j}\right), \omega^{j}\right)$, $h_{0}\left(s, x\left(s, \omega^{j}\right)\right)=J\left(s, x\left(s, \omega^{j}\right), \omega^{j}=J^{* *}\left(s, x\left(s, \omega^{j}\right), \omega^{j}\right)\right.$, which contradicts [104] for $u=u\left(t^{ \pm}\right)$, i.e. for one of these limits, as a subsequence of $s_{n}$ converges from below or above to $s$.) Define $\psi\left(s, \omega^{j}\right):=J\left(s, x\left(s, \omega^{j}\right), \omega^{j}\right)$ and define $\psi(s, \omega)=\psi\left(s, \omega^{j}\right)$ if $s \in\left[\tau_{j}, \tau_{j+1}\right)$. Now, $\psi\left(s, \omega^{j}\right)$ is continuous at each $\beta_{n}^{j}\left(\omega^{j}\right)$ (see [104]), and is hence Lipschitz continuous in $\left(\tau_{j}, T\left(\omega^{j}\right)\right)$ with 
rank

$\max \left\{\beta_{x(. .)}\left(1+\kappa_{g}\right)^{j}, \kappa_{h_{0}}\left(1+\alpha_{f}+\kappa_{f} \mid x(t, \omega)\right) \mid\right\} \leq \beta^{x(. .)}\left(1+\kappa_{g}\right)^{j}$ for some $\beta^{x(. .)}$, (by [53], [54] in Seierstad (2001)). For $s \in\left(\tau_{j}, \tau_{j+1}\right)$, define $\hat{\gamma}(s, y, u, \omega)=$ $J_{s}(s, y, \omega)+J_{y}(s, y, \omega) f(s, y, u, \omega)+$

$\lambda(s, y, u, \omega)\left\{E\left[\sup _{w \in W} J(s, y+g(s, y, V, w, \omega), \omega, s, V) \mid s, y, \omega^{j}\right]-J(s, y, \omega)\right\} \leq$ 0 , here the $C^{1}$-extension of $J$ is used when $(s, y) \in D\left(\omega^{j}\right)$, if not, $J=h_{0}$ is used. (The inequality, which holds for $(s, y) \in Q(\omega)$, follows from [101], [82].) Moreover, $\gamma(s, \omega):=\{(d / d t) E[\psi(t, \omega) \mid s, \omega]\}_{t=s+}=$ $\left[(d / d t) E[J(t, x(t ; \omega), \omega) \mid s, \omega]_{t=s+}=\hat{\gamma}(s, x(s+, \omega), u(s+, \omega), \omega) \leq 0\right.$ v.e. in $[0, T(\omega)]$, (i.e. wherever $\gamma(s, \omega)$ is defined). Write $\gamma^{*}(s, \omega)$ if $(x(., \omega), u(., \omega), T(\omega))$ is replaced by $\left(x^{*}(., \omega), u^{*}(., \omega), T^{*}(\omega)\right)$. Then, in fact, $\gamma^{*}(s, \omega)=0$ v.e., by [82],[83] and [84]. Now, Remark 29 below yields that $E h_{0}(T(\omega), x(T(\omega), \omega))=$ $E J\left(T(\omega)-, x(T(\omega)-, \omega)=E \psi(T(\omega)-, \omega)=\psi\left(0, \omega^{0}\right)+E \int_{0}^{T(\omega)} \gamma(t, \omega) d t \leq\right.$ $\psi\left(0, \omega^{0}\right)=J\left(0, x^{0}, \omega^{0}\right)=J\left(0, x^{0}, \omega^{0}\right)+E \int_{0}^{T^{*}(\omega)} \gamma^{*}(t, \omega)=$ $E J\left(T^{*}(\omega)-, x^{*}(T(\omega-, \omega), \omega)=E h_{0}\left(T^{*}(\omega), x^{*}\left(T^{*}(\omega), \omega\right)\right)\right.$

Remark 29 If $a \leq T(\omega) \leq b$ is a stopping time, and if $\phi$ is independent of $x$, (the only case we need to consider), then [89] holds for $b$ replaced by $T(\omega)$.

Proof of Remark 29 Redefine $\phi(t, \omega)$ to equal $\phi(T(\omega), \omega)$ when $t>T(\omega)$. Then $\phi$ is still nonanticipating, and $\gamma(t, \omega)=0$ for $t>T(\omega)$. Since [89] holds for the redefined $\phi$, it holds as claimed in the remark.

In the next theorem it is assumed that $\lambda$ is independent of $x$ and $u$, that $\pi$ is independent of $x$, and that $g=g\left(t, x, V_{j}, \tau_{j}, j\right)$. The function $M$ is defined in $\left[86^{* *}\right]$. Quite an impressive number of assumptions are needed in the theorem, we have collected them in A.-D. below.

\section{A. (Assumptions on $\hat{H}$ and $\hat{u})$}

Let $\phi_{k}\left(t, x, p, \omega^{j}\right), k=0, \ldots, k_{j}^{*}+2$, be $C^{1}$-functions in $(t, x, p)$ in given open sets $Q^{*}\left(\omega^{j}\right), j=1,2, \ldots$, for each $\omega^{j}$, piecewise and right-continuous in each $\tau_{i}$, each component of each $V_{i}, \tau_{i}<t$. Assume that for any $(t, x, p) \in$ $Q^{*}\left(\omega^{j}\right), \phi_{k}\left(t, x, p, \omega^{j}\right)=0$ for at most one $k$. Assume that $\hat{u}\left(t, x, p, \omega^{j}\right)$ yields the maximum denoted $\hat{H}\left(t, x, p, \omega^{j}\right)$ of $u \rightarrow H\left(t, x, u, p, \omega^{j}\right)$, for $(t, x, p)$ in $Q^{*}\left(\omega^{j}\right)$. Assume that $\hat{H}\left(t, x, p, \omega^{j}\right)$ is $C^{0} \times C^{2} \times C^{2}$ in $\left\{(t, x, p) \in Q^{*}\left(\omega^{j}\right)\right.$ : $\phi_{k}\left(t, x, p, \omega^{j}\right) \neq 0$ for all $\left.k\right\}$ and that $\hat{u}\left(t, x, p, \omega^{j}\right)$ is $C^{0} \times C^{1} \times C^{1}$ here. Assume also that for each $\omega^{j},(t, x, p) \rightarrow \hat{u}\left(t, x, p, \omega^{j}\right)$ has a $C^{0} \times C^{1} \times C^{1}$ extension to an open set containing $\operatorname{cl} A$, for any set $A$ of the form $\cap_{i} \Phi^{i}$, $\Phi^{i}=\left\{(t, x, p) \in Q^{*}\left(\omega^{j}\right): \phi_{i}\left(t, x, p, \omega^{j}\right)>0\right\}$, or $\Phi^{i}=\left\{(t, x, p) \in Q^{*}\left(\omega^{j}\right):\right.$ $\left.\phi_{i}\left(t, x, p, \omega^{j}\right)<0\right\}$, (the direction of the inequality sign may depend on $i$ ). 
B. (Existence of characteristic quadruples, and assumptions placed upon them)

Assume that a collection of sets $D^{0}\left(\omega^{j}\right) \subset\left[\tau_{j}, T_{2}\right) \times \mathbb{R}^{n}$, relatively open in $\left[\tau_{j}, T_{2}\right) \times \mathbb{R}^{n}$, and a collection of solutions $x\left(t ; s, y, \omega^{j}\right), p\left(t ; s, y, \omega^{j}\right), z\left(t ; s, y, \omega^{j}\right)$, with corresponding stopping times $T\left(s, y, \omega^{j}\right) \in\left(\max \left\{s, T_{1}\right\}, T_{2}\right]$ have been found, satisfying as functions of $t$ in $\left(s, T\left(s, y, \omega^{j}\right)\right)$, the equations

$\dot{x}=f\left(t, x, \hat{u}\left(t, x, p, \omega^{j}\right), \omega^{j}\right)$,

$\dot{p}=-p f_{x}\left(t, x, \hat{u}\left(t, x, p, \omega^{j}\right), \omega^{j}\right)+\lambda\left(t, \omega^{j}\right) p-\lambda\left(t, \omega^{j}\right) M_{x}\left(t, x, \omega^{j}\right)$,

$\dot{z}=\lambda\left(t, \omega^{j}\right) z-\lambda\left(t, \omega^{j}\right) M\left(t, x, \omega^{j}\right)$,

$z\left(T\left(s, y, \omega^{j}\right) ; s, y, \omega^{j}\right)=h_{0}\left(T\left(s, y, \omega^{j}\right), x\left(T\left(s, y, \omega^{j}\right) ; s, y, \omega^{j}\right)\right), x\left(t ; s, y, \omega^{j}\right)$ starting at $\left(s, y, \omega^{j}\right)$, for any $j$, any $\omega^{j}$, for any $(s, y) \in D^{0}\left(\omega^{j}\right)$. The sets $D^{0}\left(\omega^{j}\right)$ are assumed to satisfy the conditions that for any admissible $x(., \omega)$, $\left(s, x\left(s, \omega^{j}\right)\right) \in D^{0}\left(\omega^{j}\right)$ for all $s<T_{1}$, and that, for all $(s, y) \in D^{0}\left(\omega^{j}\right)$, $T\left(s, y, \omega^{j}\right)=\sup \left\{t^{\prime}:\left(t, x\left(t ; s, y, \omega^{j}\right)\right) \in D^{0}\left(\omega^{j}\right)\right.$ for all $\left.t \in\left[s, t^{\prime}\right]\right\}>s$, (i.e. the set is nonempty.)

It is assumed, for $(s, y) \in D^{0}\left(\omega^{j}\right)$, that $x\left(t ; s, y, \omega^{j}\right), p\left(t ; s, y, \omega^{j}\right)$ satisfies the terminal conditions [73] and the transversality condition [86*] for $T$ replaced by $T\left(s, y, \omega^{j}\right)$ and with $\Lambda_{i}\left(k, s, y, \omega^{j}\right), i=n^{\prime}+1, \ldots, n^{\prime \prime}$ continuous.

Assume, furthermore, the consistency condition [86 $\left.6^{* * *}\right]$ (for $t<T\left(s, y, \omega^{j}\right),(s, y) \in$ $\left.D^{0}\left(\omega^{j}\right)\right)$. Assume that $(s, y) \rightarrow\left(x\left(t ; s, y, \omega^{j}\right), p\left(t ; s, y, \omega^{j}\right), T\left(s, y, \omega^{j}\right)\right)$ satisfies the following differentiability conditions. Assume that for any given $\omega^{j}$, for any $(s, y) \in D^{0}\left(\omega^{j}\right)$, if

$$
\begin{aligned}
& t \in\left(s, T\left(s, y, \omega^{j}\right)\right) \text { and } \phi_{k}\left(t, x\left(t ; s, y, \omega^{j}\right), p\left(t ; s, y, \omega^{j}\right), \omega^{j}\right)=0, \text { then } \\
& \phi_{k t}+\phi_{k x}(\partial / \partial t) x\left(t^{ \pm} ; s, y, \omega^{j}\right)+\phi_{k p}(\partial / \partial t) p\left(t^{ \pm} ; s, y, \omega^{j}\right) \neq 0
\end{aligned}
$$

the partial derivatives $\phi_{k t}, \phi_{k x}$ and $\phi_{k p}$ being evaluated at $\left(t, x\left(t ; s, y, \omega^{j}\right)\right.$, $\left.p\left(t ; s, y, \omega^{j}\right), \omega^{j}\right)$. (The expression on the left side of the "nonequality" in [107] is assumed to be either $>0$, for both the right and left limit, or $<0$ for both limits.) Assume that $\left(t, x\left(t ; s, y, \omega^{j}\right), p\left(t ; s, y, \omega^{j}\right)\right)$ belongs to $Q^{*}\left(\omega^{j}\right)$, for all $t \in\left(s, T\left(s, y, \omega^{j}\right)\right)$, all $(s, y) \in D^{0}\left(\omega^{j}\right)$. Let $D^{1}\left(\omega^{j}\right):=$ $\left\{(s, y) \in D^{0}\left(\omega^{j}\right): \phi_{k}\left(s, y, p(s ; s, y), \omega^{j}\right)=0\right.$ for some $\left.k=1, \ldots, k_{j}^{*}+2\right\}$, $D_{s, y, \omega^{j}}:=\left\{t \in\left(s, T\left(s, y, \omega^{j}\right)\right): \phi_{k}\left(t, x\left(t ; s, y, \omega^{j}\right), p\left(t ; s, y, \omega^{j}\right), \omega^{j}\right)=0\right.$ for some $\left.k=1, \ldots, k_{j}^{*}+2\right\}$, and $D^{2}\left(\omega^{j}\right):=$ $\left\{(s, y) \in D^{0}\left(\omega^{j}\right): \phi_{k}\left(T\left(s, y, \omega^{j}\right), x\left(T\left(s, y, \omega^{j}\right) ; s, y, \omega^{j}\right), p\left(T\left(s, y, \omega^{j}\right) ; s, y, \omega^{j}\right), \omega^{j}\right)=\right.$ 0 for some $\left.k=1, \ldots, k_{j}^{*}+2\right\}$ and assume that for any $(s, y) \in D^{0}\left(\omega^{j}\right) \backslash$ $\left(D^{1}\left(\omega^{j}\right) \cup D^{2}\left(\omega^{j}\right)\right), s>\tau_{j}$, for any $t \notin D_{s, y, \omega^{j}}, s<t<T\left(s, y, \omega^{j}\right),\left(s^{\prime}, y^{\prime}\right) \rightarrow$ $\left(x\left(t ; s^{\prime}, y^{\prime}, \omega^{j}\right), p\left(t ; s^{\prime}, y^{\prime}, \omega^{j}\right), T\left(s^{\prime}, y^{\prime}, \omega^{j}\right)\right)$ is $C^{1}$ for all $\left(s^{\prime}, y^{\prime}\right)$ in a neighbor$\operatorname{hood} N_{t, s, y, \omega^{j}}$ of $(s, y)$. 
C. (The crossing of "fault lines" by the admissible solutions)

Define $\Phi_{k, \omega^{j}}:=\left\{(s, y) \in D^{0}\left(\omega^{j}\right): \phi_{k}\left(s, y, p(s ; s, y), \omega^{j}\right)=0\right\}$, and assume that $\Phi_{k_{j}^{*}+1, \omega^{j}}$ contains $\partial\left\{(s, y) \in D^{0}\left(\omega^{j}\right): T\left(s, y, \omega^{j}\right)>T_{1}\right\}$, and that $\Phi_{k_{j}^{*}+2, \omega^{j}}$ contains $\partial\left\{(s, y) \in D^{0}\left(\omega^{j}\right): T\left(s, y, \omega^{j}\right)<T_{2}\right\}$. By assumption, $\phi_{k_{j}^{*}+1}$ and $\phi_{k_{j}^{*}+2}$ are independent of $p$. Define $\Phi_{k^{*}+2+i, \omega^{j}}:=\left\{(s, y) \in Q\left(\omega^{j}\right)\right.$ : $\left.\phi_{i}\left(T\left(s, y, \omega^{j}\right), x\left(T\left(s, y, \omega^{j}\right) ; s, y, \omega^{j}\right), p\left(T\left(s, y, \omega^{j}\right) ; s, y, \omega^{j}\right), \omega^{j}\right)=0\right\}$. Assume that for any admissible pair $x(., \omega), T(\omega)$, for any $k=1, \ldots, 2 k^{*}+2$, for any $s<T(\omega), s>\tau_{j}$ such that $\left(s, x\left(s, \omega^{j}\right)\right)$ belongs to $\Phi_{k, \omega^{j}}$, there exists a number $\epsilon>0,\left(s^{\prime}, x\left(s^{\prime}, \omega^{j}\right)\right) \notin \Phi_{k, \omega^{j}}$ for $s^{\prime} \in(s, s+\epsilon)$. Finally, assume that [105] is satisfied for $D\left(\omega^{j}\right):=D^{0}\left(\omega^{j}\right) \cap Q\left(\omega^{j}\right)$.

D. (Assumptions on the value functions $J$ )

Assume that the following condition is satisfied. The inequality $J(s, y, \omega):=$ $z(s ; s, y, \omega)>h_{0}(s, y)$ holds for all $(s, y) \in D\left(\omega^{j}\right), s \geq T_{1}$. Assume also that [101] and [104] hold. Assume, for each admissible $x(., \omega), T(\omega)$, that $\mid J\left(t, x\left(t, \omega^{j}\right), \omega^{j}\left|\leq \alpha_{J}+\kappa_{J}\right| x\left(t, \omega^{j}\right) \mid, t \in\left(\tau_{j}, T(w)\right] \cap\left\{t:(t, x(t)) \in D^{0}(\omega)\right\}\right.$, and that $t \rightarrow J\left(t, x\left(t, \omega^{j}\right), \omega^{j}\right)$ is Lipschitz continuous in $\left(\tau_{j}, T(\omega)\right) \cap\left(s^{\prime}, s^{\prime \prime}\right)$, with rank $\hat{\kappa}_{J}\left(1+\kappa_{g}\right)^{j}$, for any interval $\left(s^{\prime}, s^{\prime \prime}\right)$ for which $\left(s, x\left(s, \omega^{j}\right)\right) \in D^{0}\left(\omega^{j}\right)$ when $s \in\left(s^{\prime}, s^{\prime \prime}\right), \alpha_{J}, \kappa_{J}, \hat{\kappa}_{J}$ perhaps dependent on $x(., \omega), T(\omega)$. Assume that $M\left(s, y, \omega^{j}\right):=E\left[\sup _{w} J\left(s, y+g\left(s, y, V, w, \omega^{j}\right), \omega^{j}, s, V\right) \mid s, \omega^{j}\right]$ is $C^{0} \times C^{2}$ in $D^{0}\left(\omega^{j}\right) \backslash\left(D^{1}\left(\omega^{j}\right) \cup D^{2}\left(\omega^{j}\right)\right)$, and that for any $(s, y) \in D^{0}\left(\omega^{j}\right) \backslash\left(D^{1}\left(\omega^{j}\right) \cup\right.$ $\left.D^{2}\left(\omega^{j}\right)\right), M\left(s, y, \omega^{j}\right)$ has a $C^{0} \times C^{2}$-extension to an open set around the set $\left\{\left(t, x\left(t ; s, y, \omega^{j}\right)\right): t \in[a, b]\right\}$, for any interval $[a, b] \subset\left[0, T\left(s, y, \omega^{j}\right)\right)$ for which $(a, b) \cap D_{s, y, \omega^{j}}=\emptyset$. Assume also that $\sup _{t \in\left[s, T\left(s, y, \omega^{j}\right)\right) \backslash Q_{s, y, \omega^{j}}}\left|M\left(t, x\left(t ; s, y, \omega^{j}\right), \omega^{j}\right)\right|$,

$\sup _{t \in\left[s, T\left(s, y, \omega^{j}\right)\right) \backslash Q_{s, y, \omega^{j}}}\left|M_{x}\left(t, x\left(t ; s, y, \omega^{j}\right), \omega^{j}\right)\right|$ and

$\sup _{t \in\left[s, T\left(s, y, \omega^{j}\right)\right) \backslash Q_{s, y, \omega^{j}}}\left|M_{x x}\left(t, x\left(t ; s, y, \omega^{j}\right), \omega^{j}\right)\right|$ are locally bounded on $D^{0}\left(\omega^{j}\right) \backslash$ $\left(D^{1}\left(\omega^{j}\right) \cup D^{2}\left(\omega^{j}\right)\right)$.

\section{Theorem 16 (Sufficient condition for characteristic quadruples )}

Assume that A.-D. above are satisfied. Assume, furthermore, that $\hat{w}\left(t, x, v, \omega^{j}\right)$ gives maximum of $J\left(t, x+g\left(t, x, v, w, \omega^{j}\right), \omega^{j}, t, v\right)$ for $(t, x) \in D^{0}\left(\omega^{j}\right)$. Assume, moreover, that $\eta^{*}\left(T\left(s, y, \omega^{j}\right), \omega^{j}\right)=0$ if $T\left(s, y, \omega^{j}\right) \in\left(\max \left\{s, T_{1}\right\}, T_{2}\right),(s, y) \in$ $D^{0}\left(\omega^{j}\right)$, where $\eta^{*}\left(T, \omega^{j}\right):=\eta\left(T, x\left(T ; s, y, \omega^{j}\right), p\left(T ; s, y, \omega^{j}\right), \omega^{j}\right), \eta\left(T, x, p, \omega^{j}\right):=$

$\hat{H}\left(T, x, p, \omega^{j}\right)+h_{0 T}(T, x)+\lambda\left(T, \omega^{j}\right)\left\{E\left[\sup _{w} J\left(T, x+g\left(T, x, V, w, \omega^{j}\right), \omega^{j}, T, V\right) \mid T, \omega^{j}\right]-\right.$ $\left.h_{0}(T, x)\right\}$.

Define $T\left(s, y, \omega^{j}\right)=s$ if $(s, y) \in Q\left(\omega^{j}\right) \backslash D\left(\omega^{j}\right)$ and define $x(t, \omega)=x\left(t, 0, x^{0}, \omega^{0}\right)$, 
for $t \in\left[0, \tau_{1}\right] \cap\left[0, T\left(0, x^{0}, \omega^{0}\right)\right]$, and by induction, generally, $x(t, \omega)=x\left(t, \tau_{k}, x\left(\tau_{k}, \omega\right)+g\left(\tau_{k}, x\left(\tau_{k}, \omega\right), w\left(\tau_{k}, x\left(\tau_{k}, \omega\right), v_{k}, \omega^{k-1}\right), \omega^{k-1}\right)\right)$, for $t \in\left(\tau_{k}, \tau_{k+1}\right] \cap\left[0, T\left(\tau_{k}, x\left(\tau_{k}, \omega\right)+g\left(\tau_{k}, x\left(\tau_{k}, \omega\right), \hat{w}\left(\tau_{k}, x\left(\tau_{k}, \omega\right), v_{k}, \omega^{k-1}\right), \omega^{k-1}\right)\right)\right]$. Assume, finally, that $\tilde{w}\left(t, v, \omega^{j}\right):=\hat{w}\left(t, x(t ; \omega), v, \omega^{j}\right)$ satisfies the standard requirements on such controls described above. Define $\tilde{u}\left(t, \omega^{j}\right)=\hat{u}\left(t, x(t ; \omega), p(t ; \omega), \omega^{j}\right)$, $\tilde{T}(t, \omega):=T\left(t, x(t, \omega), \omega^{j}\right)$ if $t \in\left(\tau_{j}, \tau_{j+1}\right]$. Then $\tilde{u}(t, \omega), \tilde{w}(t, v, \omega), \tilde{T}(t, \omega)$ is an optimal triple.

Proof of Theorem 16: For simplicity, in the proof, a problem is considered in which the control $w$ does not appear. Let $(s, y) \in D_{0}\left(\omega^{j}\right):=$ $D^{0}\left(\omega^{j}\right) \backslash\left(D^{1}\left(\omega^{j}\right) \cup D^{2}\left(\omega^{j}\right)\right)$. Choose a $t$ close to $T\left(s, y, \omega^{j}\right)$, such that $\left[t, T\left(s, y, \omega^{j}\right)\right) \subset \complement D_{s, y, \omega^{j}}$. Recall that, for $Y=Z=(s, y), z\left(t ; s, y, \omega^{j}\right)=$ $\psi(t, Z, Y):=\int_{t}^{T\left(Z, \omega^{j}\right)} \lambda\left(\rho, \omega^{j}\right) e^{\int_{\rho}^{t} \lambda\left(\xi, \omega^{j}\right) d \xi} M\left(\rho, x\left(\rho ; Y, \omega^{j}\right), \omega^{j}\right) d \rho+$ $h_{0}\left(T\left(Z, \omega^{j}\right), x\left(T\left(Z, \omega^{j}\right), Y, \omega^{j}\right)\right) e^{\int_{T(Z)}^{t} \lambda\left(\rho, \omega^{j}\right) d \rho}$. Differentiating with respect to $Z$ yields $\psi_{Z}(t, Z, Y)=$

$\left.\lambda\left(T\left(Z, \omega^{j}\right), \omega^{j}\right) M\left(T\left(Z, \omega^{j}\right), x\left(T\left(Z, \omega^{j}\right), Y, \omega^{j}\right), \omega^{j}\right)\right) e^{\int_{T\left(Z, \omega^{j}\right)}^{t} \lambda\left(\rho, \omega^{j}\right) d \rho} T^{\prime}$ $\left.+h_{0 t}\left(T\left(Z, \omega^{j}\right), x\left(T\left(Z, \omega^{j}\right), Y, \omega^{j}\right)\right)\right) e^{\int_{T\left(Z, \omega^{j}\right)}^{t} \lambda\left(\rho, \omega^{j}\right) d \rho} T^{\prime}+$ $\left.h_{0 x}\left(T\left(Z, \omega^{j}\right), x\left(T\left(Z, \omega^{j}\right), Y, \omega^{j}\right)\right) \dot{x}\left(T\left(Z, \omega^{j}\right), Y, \omega^{j}\right)\right) e^{\int_{T\left(Z, \omega^{j}\right)}^{t} \lambda\left(\rho, \omega^{j}\right) d \rho} T^{\prime}-$ $\lambda\left(T\left(Z, \omega^{j}\right), \omega^{j}\right) h_{0}\left(T\left(Z, \omega^{j}\right), x\left(T\left(Z, \omega^{j}\right), Y, \omega^{j}\right)\right) e^{\int_{T\left(Z, \omega^{j}\right)}^{t} \lambda\left(\rho, \omega^{j}\right) d \rho} T^{\prime}$. (Note that the local boundedness of $M, M_{x}$ and $M_{x x}$ yields that $x\left(t ; s, y, \omega^{j}\right)$ and $x_{y}\left(t ; s, y, \omega^{j}\right)$ are locally bounded, that $\psi(t, Z, Y)$ and that the derivatives with respect to $Z$ - and later on with respect to $Y$ - can be calculated in the manner done. (Formally when, say $Y$, changes, the interval of definition $\left[t, T\left(Z, \omega^{j}\right)\right.$ ) also changes, as $Y=Z$ ).)

Now, $\left(g^{k, j}\left(T\left(Z, \omega^{j}\right), x\left(T\left(Z, \omega^{j}\right), \omega^{j}\right)\right)_{i}=\hat{x}_{i}\right.$ for $\Lambda_{i}\left(k, Z, \omega^{j}\right)>0, i=n^{\prime}+$ $1, \ldots, n^{\prime \prime}$, and also for $i \leq n^{\prime}$. Thus, similar to what was obtained in the proof of Lemma 3, the equality in fact holds also for all $Z^{\prime}$ close to $Z$, so, (still for $(s, y)=Z=Y)$, $p\left(T(Z, \omega), Z, \omega^{j}\right)(d / d Z) x\left(T\left(Z, \omega^{j}\right), Z, \omega^{j}\right)=$ $h_{0 x}\left(T\left(Z, \omega^{j}\right), x\left(T\left(Z, \omega^{j}\right), Y, \omega^{j}\right)\right)(d / d Z) x\left(T\left(Z, \omega^{j}\right), Z, \omega^{j}\right)$, i.e. $p\left(T(Z, \omega), Z, \omega^{j}\right)\left[\dot{x}\left(T\left(Z, \omega^{j}\right), Y, \omega^{j}\right) T^{\prime}+x_{Y}\left(T\left(Z, \omega^{j}\right), Y, \omega^{j}\right)\right]=$ $h_{0 x}\left(T\left(Z, \omega^{j}\right), x\left(T\left(Z, \omega^{j}\right), Y, \omega^{j}\right)\right)\left[\dot{x}\left(T\left(Z, \omega^{j}\right), Y, \omega^{j}\right) T^{\prime}+x_{Y}\left(T\left(Z, \omega^{j}\right), Y, \omega^{j}\right)\right]$. For $t=T\left(Z, \omega^{j}\right)$, replacing $h_{0 x} \dot{x} T^{\prime}$ in the expression for $\psi_{Z}$ by $p \dot{x} T^{\prime}+p x_{Y}-h_{0 x} x_{Y}$, (we here use a shorthand notation), yields $\psi_{Z}=p x_{Y}+\eta^{*} T^{\prime}-h_{0 x} x_{Y}$. Hence, for $t=T\left(Z, \omega^{j}\right), \psi_{Z}\left(T\left(Z, \omega^{j}\right), Z, Y\right)=$ $p\left(T\left(Z, \omega^{j}\right), Z, \omega^{j}\right) x_{Y}\left(T\left(Z, \omega^{j}\right), Y, \omega^{j}\right)-$ $h_{0 x}\left(T\left(Z, \omega^{j}\right), x\left(T\left(Z, \omega^{j}\right), Y, \omega^{j}\right)\right) x_{Y}\left(T\left(Z, \omega^{j}\right), Y, \omega^{j}\right)$ at points $(s, y)$ for which 
$T\left(s, y, \omega^{j}\right) \in\left(T_{1}, T_{2}\right)$, by the $C^{1}$-property of $T\left(s, y, \omega^{j}\right)$ and the fact that then $\eta^{*}=0$. The equality $(0=) \psi_{Z}\left(T\left(Z, \omega^{j}\right), Z, Y\right)=$ $p\left(T\left(Z, \omega^{j}\right), Z, \omega^{j}\right) x_{Y}\left(T\left(Z, \omega^{j}\right), Y, \omega^{j}\right)-$

$h_{0 x}\left(x\left(T\left(Z, \omega^{j}\right), Y, \omega^{j}\right)\right) x_{Y}\left(T\left(Z, \omega^{j}\right), Y \omega^{j}\right)$ evidently holds at points $(s, y)$ for which there exist a ball around $(s, y)$ in which $T\left(s, y, \omega^{j}\right)$ is constant equal to $T_{1}$ or $T_{2}$, since then $T^{\prime}=0$. All points $(s, y)$ in $D_{0}\left(\omega^{j}\right)$ exhibits one of these two types of behaviour. Since $\psi_{Y}(T(Z, \omega), Z, Y)=h_{0 x} x_{Y}$, then $\left[(d / d Y) z\left(t ; Y, \omega^{j}\right)\right]_{t=T\left(Z, \omega^{j}\right)}=\psi_{Z}+\psi_{Y}=\left[p\left(t ; Y, \omega^{j}\right) x_{Y}\left(t ; Y, \omega^{j}\right)\right]_{t=T\left(Z, \omega^{j}\right)}$. Then, as in the proofs of Lemma 3 , Lemma 7 , using the transformation in the proof of Lemma $5, w_{t}=0, \hat{w}_{t}=0$, where $w(t, s, y)=\left[z_{y}\left(t ; s, y, \omega^{j}\right)-p\left(t ; s, y, \omega^{j}\right) x_{y}\left(t ; s, y, \omega^{j}\right)\right] e^{\int_{T}^{t}-\lambda\left(\rho, \omega^{j}\right) d \rho}$, $\hat{w}(t)=\left[z_{s}\left(t ; s, y, \omega^{j}\right)-p\left(t ; s, y, \omega^{j}\right) x_{s}\left(t ; s, y, \omega^{j}\right)\right] e^{\int_{T}^{t}-\lambda\left(\rho, \omega^{j}\right) d \rho}, T=T\left(Z, \omega^{j}\right)$, ( $Z$ fixed), so $w(t, s, y)=0, \hat{w}(t, s, y)=0$. In fact, $J\left(s, y, \omega^{j}\right)=z\left(s ; s, y, \omega^{j}\right)$ is $C^{1}$ and satisfies the HJB-equation in $D^{0}\left(\omega^{j}\right) \backslash\left(D^{1}\left(\omega^{j}\right) \cup D^{2}\left(\omega^{j}\right)\right)$. Then, evidently Theorem 16 follows from Theorem 15, (see also Lemma 8).

\section{Appendix}

[A]. The probability of one or more jumps in $(t, t+\Delta t)$ is $1-\exp \left(\int_{t}^{t+\Delta t} \lambda\left(s, \omega^{k}\right) d s\right) \approx \lambda\left(t+, \omega^{k}\right) \Delta t$. Given that one jump $\tau_{k+1}$ has occurred in $(t, t+\Delta t)$, the probability for further jumps is $1-\exp \left(\int_{\tau_{k+1}}^{t+\Delta t} \lambda\left(s, \omega^{k+1}\right) d s\right)$. The expected value of $\phi$ resulting from these jumps, given that one or more of them occur, is bounded. This follows from [54], [53] in Seierstad (2001) and the existence of the constants $\alpha_{\phi}, \kappa_{\phi}$. Altogether, the case of two or more jumps leads to a second order term.

[B] By [53],54] in Seierstad (2001), for some $K^{\prime}$ independent of $i,\left|\phi\left(\tau_{i}, x\left(\tau_{i}^{ \pm}, \omega\right), \omega\right)\right| \leq$ $\alpha_{x(. .)}+\kappa_{x(. .)} K^{\prime}\left(1+\kappa_{g}\right)^{i} \leq \tilde{\kappa}\left(1+\kappa_{g}\right)^{i}$, where $\tilde{\kappa}=\alpha_{x(. . .)}+\kappa_{x(. . .)} K^{\prime}$. Given any interval $\left[s, s^{\prime}\right]$, then, for some $K$,

$\operatorname{Pr}\left[\right.$ one or more jumps in $\left.\left[s, s^{\prime}\right] \mid \tau_{j}<s, \tau_{j+1} \geq s\right] \leq K\left|s^{\prime}-s\right|$,

due to the assumptions on $\lambda($.$) . Given that \tau_{j+1}, \ldots, \tau_{k}$ belongs to $\left(s, s^{\prime}\right)$, $\left|\phi\left(s^{\prime}, x\left(s^{\prime}, \omega\right), \omega\right)-\phi(s, x(s, \omega), \omega)\right| \leq \hat{\kappa}_{x(. .)}\left(1+\kappa_{g}\right)^{j}\left|\tau_{j}-s\right|+\sum_{j \leq i<k} \hat{\kappa}_{x(., .)}(1+$ $\left.\left.\kappa_{g}\right)^{i} \mid \tau_{i+1}-\tau_{i}\right)\left|+\hat{\kappa}_{x(., .)}\left(1+\kappa_{g}\right)^{k}\right| s^{\prime}-\tau_{k}\left|+\sum_{j \leq i \leq k}\right| \phi\left(\tau_{i}, x\left(\tau_{i}^{+}, \omega\right), \omega\right)-\phi\left(\tau_{i}, x\left(\tau_{i}^{-}, \omega\right), \omega\right) \mid \leq$ $2 \hat{\kappa}_{x(.,)}\left(1+\kappa_{g}\right)^{k} T+2 \tilde{\kappa}_{x(. .)}\left(1+\kappa_{g}\right)^{k}=: \Lambda_{k}$. Now, $\sum_{k} \operatorname{Pr}\left[\tau_{j+1}, \ldots, \tau_{k}\right.$ belongs to $\left.\left(s, s^{\prime}\right) \mid \tau_{j}<s, \tau_{j+1} \geq s\right] \Lambda_{k}=: \xi<\infty$. Moreover, $E\left[\mid \phi\left(s^{\prime}, x\left(s^{\prime}, \omega\right), \omega\right)-\right.$ $\left.\phi(s, x(s, \omega), \omega) \| \tau_{j}<s, \tau_{j+1} \geq s\right] \leq \operatorname{Pr}\left[\right.$ one or more jumps in $\left[s, s^{\prime}\right] \mid \tau_{j}<$ $\left.s, \tau_{j+1} \geq s\right] \xi+\operatorname{Pr}\left[\tau_{j+1} \notin\left[s, s^{\prime}\right] \mid \tau_{j}<s, \tau_{j+1} \geq s\right] \hat{\kappa}_{x(. . .)}\left(1+\kappa_{g}\right)^{j}\left|s^{\prime}-s\right| \leq$ $K\left(s^{\prime}-s\right) \xi+\hat{\kappa}_{x(. .)}\left(1+\kappa_{g}\right)^{j}\left|s^{\prime}-s\right|$.

Note that the Lipschitz condition on $\phi$ follows from the following condi- 
tions: $\phi_{1}(s, x(s, \omega), \omega) \leq \alpha^{x(. .)}+\kappa^{x(. .)}|x(s, \omega)|, \phi_{2}(s, x(s, \omega), \omega) \leq \bar{\kappa}^{x(. .)}$. (Use [53],[54] in Seierstad (2001), $(d / d s) \phi=\phi_{1}+\phi_{2} \dot{x}$, and [19].)

Lemma 10. Let $\phi_{i}(t, v), i=1, \ldots, i^{*}$, be $C^{1}$. Let $\Phi_{+}^{i}:=\left\{(t, v): \phi_{i}(t, v)>0\right\}$, let $\Phi_{-}^{i}:=\left\{(t, v): \phi_{i}(t, v)<0\right\}$. Let $\mathcal{A}$ be the collection of sets $A:=$ $\Phi^{1} \cap \Phi^{2} \ldots \cap \Phi^{i^{*}}$, where each $\Phi^{i}$ is $\Phi_{+}^{i}$ or $\Phi_{-}^{i}$, (whether a minus sign or a plus sign occurs, may depend on $i$ ). Assume that for any point $(t, v)$, for at most one $i, \phi_{i}(t, v)=0$. Assume that $h(t, v)$ is a given function such that, for each $A$ in $\mathcal{A},\left.h(t, v)\right|_{A}$ has an extension $h^{A}(t, v)$ to $\operatorname{cl} A$ that is $C^{0} \times C^{1}$. Assume that, for all $A$ in $\mathcal{A}$, if $\phi_{i}(t, v)=0$, then $\phi_{i t}+\phi_{i v} h^{A}(t, v)>0$ for both $A=\left(\cap_{j \neq i} \Phi^{j}\right) \cap \Phi_{-}^{i}$ and $A=\left(\cap_{j \neq i} \Phi^{j}\right) \cap \Phi_{+}^{i}$, or the opposite strict inequality holds for these two sets $A$.

Write $z=(s, w)$ and consider the equation

$$
\dot{v}=h(t, v), v(s)=w .
$$

Then the following conclusions hold: Assume that, for any point $z=\hat{z}=$ $(\hat{s}, \hat{w})$ belonging to some $A$ in $\mathcal{A}$, the solution $v(t, \hat{z})$ exists on $[\hat{s}, T]$. Then for $z=(s, w)$ close to $\hat{z}$, the solution $v(t, z)$ of [108] exists on $[\hat{s}, T]$, and, for $t \notin$ $\left\{t: \phi_{i}(t, v(t, \hat{z}))=0\right.$, for some $\left.i\right\}, t>\hat{s}$, near $\hat{z}, z \rightarrow v(t, z)$ is continuously differentiable. For such $t,(\partial / \partial w) v(t, \hat{s}, \hat{w})$ is invertible. If the equation in [108] satisfies standard global growth conditions, say $|h(t, 0)|,\left|h_{v}(t, v)\right| \leq a(t)$, (wherever $h_{v}$ exists), a(t) continuous, then for any initial point $z=(s, w)$, a solution $v(t, z)$ exists on any given interval $[s, T]$.

Proof Let $\hat{z}=(\hat{s}, \hat{w})$ be a given point in some set $A$ in $\mathcal{A}$. We shall consider only the case of one single function $\phi$, and only one "crossing point" $t=t(\hat{z})$ in $(\hat{s}, T)$, where $\phi(t, v(t, \hat{z}))=0$, the general case being a trivial extension. Thus, assume that $\hat{z}=(\hat{s}, \hat{w})$ belongs to one of the sets $A$ in $\mathcal{A}=\{(t, y): \phi(t, y)<0\},\{(t, y): \phi(t, y)>0\}\}$. Then $\left(t^{\prime \prime}, v\left(t^{\prime \prime}, \hat{z}\right)\right)$ belongs to the other set $A^{\prime} \in \mathcal{A}$ for $t^{\prime \prime}>t(\hat{z})$, by the non-tangentiallity condition. Let $h^{A}$ be a $C^{0} \times C^{1}$-extension of $\left.h\right|_{A}$ to an open set larger that $\operatorname{cl} A$. Let $v^{A}(t, z)$ be the solution of $\dot{v}=h^{A}(t, v), v(s)=w$, for $z$ close to $\hat{z}$. It can be assumed that $v^{A}(t, z)$ is defined on some interval $[a, b], a<\hat{s}, b>t(\hat{z})$, and the solution depends continuously on $(s, w)$ near $\hat{z}, s \in[a, b]$. For $z$ near $\hat{z}$, let $t(z)$ be the first time $v^{A}(t, z)$ hits the surface $S=\{(t, x) ; \phi(t, x)=0\}$. By the non-tangential approach of $v^{A}(t, \hat{z})$ to the surface $S, t(z)$ is close to $t(\hat{z})$, and $v^{A}(t(z), z)$ is close to $v(t(\hat{z}), \hat{z})$. In fact, by the nontangentiallity conditions, $t(z)$ is $C^{1}$ for $z$ close to $\hat{z}$. Now, $(t, v(t, \hat{z}))$ belongs to $A^{\prime}$ for $t>t(\hat{z})$. Let $h^{A^{\prime}}$ be a $C_{ \pm}^{0} \times C^{1}$-extension of $\left.h\right|_{A^{\prime}}$ to an open set larger that $\operatorname{cl} A^{\prime}$. Let $v^{A^{\prime}}\left(t, z^{\prime}\right)$ be the unique solution of $\dot{v}=h^{A^{\prime}}(t, v), v\left(s^{\prime}\right)=w^{\prime}$, for $z^{\prime}=\left(s^{\prime}, w^{\prime}\right)$ 
close to $\tilde{z}=(t(\hat{z}), v(t(\hat{z}), \hat{z})$. (For such $z$, the solution does exist on an interval $\left[a^{\prime}, b^{\prime}\right], a^{\prime}<t(\hat{z}), b^{\prime}>T$.) Moreover, $v^{A^{\prime}}\left(t, z^{\prime}\right)$ is continuous in $z^{\prime}$ near $\tilde{z}$. In particular, $v^{A^{\prime}}\left(t, z^{\prime}\right)$ exists for $z^{\prime}=z^{\prime}(z)=\left(t(z), v^{A}(t(z), z)\right)$, z close to $\hat{z}$, and by the non-tangential departure from $S,\left(t, v^{A^{\prime}}\left(t, z^{\prime}(z)\right)\right.$ belongs to $A^{\prime}$, for $t>t(z), t$ close to $t(z)$. In fact, for $z$ close to $\hat{z},\left(t, v^{A^{\prime}}\left(t, z^{\prime}(z)\right)\right.$ belongs to $A^{\prime}$ for all $t \in(t(z), T]$, and $v^{A^{\prime}}\left(t, z^{\prime}(z)\right)$ is $C^{1}$ in $z$ near $\hat{z}$ for such $t$. Define $v(t, z)=v^{A}(t, z)$, for $t \in(a, t(z)], v(t, z)=v^{A^{\prime}}\left(t, t(z), v^{A}(t(z), z)\right)$, for $t>t(z)$, which is a unique solution of [108], (due to the extension property), $C^{1}$ in $z$ near $\hat{z}$. Reversing time, for any $\bar{t}>t(\hat{z})$, there is a unique solution $\bar{v}\left(t^{\prime} ; z^{\prime \prime}\right), z^{\prime \prime}=(t, w), z^{\prime \prime}$ near $\bar{z}=(\bar{t}, v(\bar{t} ; \hat{z}))$, defined for $t^{\prime}$ in an interval $\left[a^{\prime \prime}, b^{\prime \prime}\right], a^{\prime \prime}<\hat{s}, b^{\prime \prime}>\bar{t}$, being $C^{1}$ in $z^{\prime \prime}$ near $\bar{z}$. Now, $w=\bar{v}(t, \bar{t}, v(\bar{t},(t, w))$, which means that $I=\bar{v}_{3} v_{3}$, i.e. the derivative $v_{3}$ is invertible for $(t, w)$ close to $\hat{z}$.

\section{Exercises}

Exercise 1 Add the possibility of a second jump in Example 1, both for scrap value $a x(T)$ and $a x(T)^{2} / 2$. Solve the problems as far as possible. (Hint: Now $x(t ; s, y, 1), x(t ; s, y, 0)$ becomes $x(t ; s, y, 2), x(t ; s, y, 1)$ etc. Even in the case of $a x(T)^{2} / 2$, we have an explicit formula for $p(t ; s, y, 1)$, as $\dot{x}=p$, and $\dot{x}(t ; s, y, 1)$ is known. The reader might like to put $a=2$.) Does it change the optimal control in Example 1, if instead of a unit jump, we assume that the jump is a stochastic variable $V$, with finite expectation?

Exercise 2 Consider the piecewise deterministic problem max $E\left[\int_{0}^{T} \sqrt{ } u d t+\right.$ $x(T)], \dot{x}=x-u, u \in \mathbb{R}$ and $x(\tau+)-x(\tau-)=x(\tau-)$. Two jumps can occur with intensity $\lambda$. Solve the problem.

Exercise 3 Consider the piecewise deterministic problem max $E\left[\int_{0}^{T}-\left(u^{2} / 2\right) d t+\right.$ $x(T)]$, subject to $\dot{x}=u, x(0)=0, u \in \mathbb{R}$, and $x(\tau+)-x(\tau-)=-(x(\tau-))^{2}$. A single jump can occur with intensity $\lambda$. Solve the problem. (Hint: To find $x(t ; s, y, 0)$ for $(s, y)=(0,0)$, construct a second order equation for this function).

Exercise 4 Solve the piecewise deterministic problem $\max E\left\{\int_{0}^{1} \ln u d t+x(1)\right\} d x / d t=-u, \in u \in(0, \infty), x_{0}=2$,

where $x(t)$ can jump upwards at most $N$ times, all the times with the same intensity $\lambda$. At the jump times $\tau$, the state changes according to 
$x(\tau+)-x(\tau-)=x(\tau-)$.

a. Find the optimal controls $u(t ; s, y, j)$ for $j=N, N-1, N-2$.

b. Try to find (a description of) the optimal controls $u(t ; s, y, N-k)$ for general $k=1,2, \ldots$

Solution Generally, $u(t, j)=1 / p(t, j)$. (Neither $p$ nor $u$ depend on the starting point $(t, x))$. Now, $p(t, N)=1$. Consider $\dot{p}(t, N-1):=\lambda p-\lambda 2$. Evidently, $p(t)=2-e^{\lambda(t-1)}$, (recall $\left.p(1)=1\right)$. Next, $\dot{p}(t, N-2):=$ $\lambda p-\lambda 2\left(-e^{\lambda(t-1)}+2\right)$ so, $p(t, N-2)=4-3 e^{\lambda(t-1)}-2 e^{\lambda(t-1)}(t-1)$. Writing $p(t, N-k)=a_{k}+a_{k, 0} e^{\lambda(t-1)}+e^{\lambda(t-1)} a_{k, 1}(t-1)+\ldots e^{\lambda(t-1)} a_{k, k-1}(t-1)^{k-1}$, one easily calculates $p(t, N-(k+1))$, and hence the difference equations for the coefficients. In fact, $a_{k+1}=2 a_{k}, a_{k, 0}=1-2 a_{k}$, and for $i>0, a_{k+1, i}=2 a_{k, i-1} / i$.

Exercise 5 Consider the problem $\max \int_{0}^{T} u^{\gamma} d t+a x(T), \dot{x}=d x-u, x(0)=c$, $0<\gamma<1$. With intensity $\lambda, x$ may experience a single upwards jump of size $b$. Find $x(t ; s, y, 1)$ and try to derive a second order equation determining the solution $x(t ; 0, c, 0)$. (Interpretation: $x$ is wealth, which gives interest earnings $d x, u$ is consumption, we search for, and may find, an oil field increasing our wealth by $b$.) Drop the term $a x(T)$ and instead require $x(T) \geq 0$, and do the same in this case.)

Exercise $6 \max \int_{0}^{2} x d t, \dot{x}=u \in[-1,1], x(0)=0, x(2) \leq 1$, where $x$ can have a single upward jump of one unit, with intensity $\lambda$. Hint: As long as no jump has occurred, we have to operate with the constraint $x(2) \leq 0$, otherwise $x(2) \leq 1$ may be violated, as a jump can occur arbitrary close to $t=2$.

Solution If we start in $(s, y)$, the after-a-jump-adjoint function $p(t ; s, y, 1)=$ $(3+s-y) / 2-t,\left(u=-1\right.$ in $\left(\sigma^{\prime}, 2\right)$, where $\left.\left.\sigma^{\prime}=3+s-y\right)\right)$. Before a jump, we only seek the solution $x(t)=x(t ; 0,0,0)$. Now, $p(t)=p(t ; 0,0,0)$ satisfies $\dot{p}(t)=-1-\lambda p(t, t, x(t)+1,1)+\lambda p=-1-\lambda(3-x(t)-1-t) / 2+\lambda p$, so $p(t)=h e^{\lambda t}+1 / \lambda-e^{\lambda t} \int_{t}^{2} \lambda e^{-\lambda z}[(3-x(z)-1-z) / 2] d z$, where $h$ a constant such that $\leq h e^{\lambda 2}+1 / \lambda=p(2) \leq 0$, (so $\left.h<0\right)$. Now, $x(z) \leq 2-z,(x(z)$ cannot come above a line through $(0,2)$ with slope -1 , given by $2-z)$. Hence, $(3-x(z)-1-z) / 2 \geq(3-(2-z)-1-z) / 2=0$. As $p(2) \leq 0$, the differential equation for $p$ gives that $p(t)$ stays nonpositive when going backwards, and in fact $\dot{p}(t)$ stays negative. As $p(t)$ is strictly decreasing, $u=1$ for $t<\rho$, $u=-1$ for $t>\rho$. Using $x(0)=0, x(2)=0$ we must have $\rho=1$. The solution is reasonably enough independent of $\lambda$. 
Exercise 7 Solve

$$
\left[\int_{0}^{1 / 2}(1-u) d t+(x(1 / 2))^{2} / 2\right] \dot{x}=u \in[0,1], x(0)=1 / 4, x(1 / 2) \text { free, }
$$

where a single unit upwards jump in the state $x$ may occur with intensity $\lambda=2$. (Hint: Note that a switch point for $u$ before the jump will be given by an unsolvable equation.)

Exercise 8 Solve the optimal stopping problem

$\max E\left[\int_{0}^{T}(x-t) d t\right], \dot{x}=0, x(1)=1, T \in[0,2]$, when $x$ can have one unit jump upwards with intensity $\lambda$.

\section{References}

Costa, O.L.V, Raymundo, C.A.D., Dufor, F. (2000), Optimal stopping with continuous control of piecewise deterministic Markov processes, Stochastic Reports 70, 41-73.

Costa, O.L.V, Raymundo, C.A.D. (2000), Impulse and continuous control of piecewise deterministic Markov processes, Stochastic Reports 70, 75-107.

Davis, M.H.A (1993) Markov Models and Optimization, Chapman and Hall, London.

Davis, M.H.A., Farid, M. (1999), Piecewise deterministic processes and viscosity solutions, McEneaney, W.M.(ed) et al., Stochastic analysis, control, optimization and applications, A volume in honour of W.H.Fleming, on occasion of his 70th birthday. Birkhauser, 249-268.

Seierstad, A. Necessary conditions and sufficient conditions for optimal control of piecewise deterministic control systems, Memorandum No 5/2001, Dept. of Economics, University of Oslo, Norway.

Seierstad, A. Maximum principle for stochastic control in continuous time with hard end constraints. Memorandum No 24/2002, Dept. of Economics, University of Oslo, Norway

Seierstad, A. (1998), Fields of extremals and infinite horizon optimal con- 
trol problems, Optimal Control, Applications and Methods, 19, 377-392.

Seierstad, A.(1997) Dynamic programming approach to infinite horizon deterministic continuous time control problems, Memorandum no. 15/1997 from Dept. of Economics, University of Oslo, no.

See also references in Seierstad (2001) 